\title{
The Krichever Map, Vector Bundles Over Algebraic Curves, and Heisenberg Algebras ${ }^{\star}$
}

\author{
M.R. Adams ${ }^{1}$ and M.J. Bergvelt ${ }^{2}$ \\ 1 Department of Mathematics, University of Georgia, Athens, Georgia 30602, USA; \\ email:adams@joe.math.uga.edu \\ 2 Department of Mathematics, University of Illinois, Urbana, IL 61801, USA; \\ email:bergv@huygens.math.uiuc.edu
}

Received January 2, 1992; in revised form October 1, 1992

\begin{abstract}
We study the Grassmannian $G r_{X}^{n}$ consisting of equivalence classes of rank $n$ algebraic vector bundles over a Riemann surface $X$ with an holomorphic trivialization at a fixed point $p$. Commutative subalgebras of $g l\left(n, H_{\lambda}\right), H_{\lambda}$ being the ring of functions holomorphic on a punctured disc about $p$, define flows on the Grassmannian, giving rise to classes of solutions to multi-component KP hierarchies. These commutative subalgebras correspond to Heisenberg algebras in the Kac-Moody algebra associated to $g l\left(n, H_{\lambda}\right)$. One can obtain, by the Krichever map, points of $G r_{X}^{n}$ (and solutions of mcKP) from coverings $f: Y \rightarrow X$ and other geometric data. Conversely for every point of $G r_{X}^{n}$ and for every choice of Heisenberg algebra we construct, using the cotangent bundle of $G r_{X}^{n}$, an algebraic curve covering $X$ and other data, thus inverting the Krichever map. We show the explicit relation between the choice of Heisenberg algebra and the geometry of the covering space.
\end{abstract}

\section{Introduction}

1.1. In the seventies it was discovered that one could obtain solutions of certain non-linear evolutionary equations of "soliton type" in terms of $\theta$-functions of Riemann surfaces or, more generally, one could construct solutions from coherent sheaves on algebraic curves, see e.g., [Kr, Mum1, Du, KrN, vMM]. (In fact, certain solutions of the Korteweg-de Vries equation $(\mathrm{KdV})$ in terms of elliptic functions were known classically [KdV].) Somewhat later it was discovered that solutions of such equations could be identified with points of infinite dimensional Grassmannians, or, equivalently, with the orbits of infinite dimensional groups in representation spaces (see e.g., [Sa, DaJKM, Ka]).

Both points of view, the algebro-geometric one and the representation theoretic, could be connected by associating to a Riemann surface (along with a line

* The research was partially supported by US Army grant DAA L03-87-K-0110 and NSF grant DMS 9106938 
bundle and other ingredients that won't concern us for the moment) a point of an infinite dimensional Grassmannian, [SeW]. This map from algebrogeometric data to Grassmannians we will call (as is now standard) the Krichever map.

In the representation theoretic approach one starts with, say, an affine, simply laced, Kac-Moody algebra $\hat{\mathrm{g}}$ and a highest weight representation (see $[\mathrm{Ka}])$. For every maximal Heisenberg algebra $\hat{\mathscr{H}}$ in $\hat{\mathrm{g}}$ one has an explicit construction of the basic representation in terms of vertex operators, certain formal infinite order differential operators in an infinite number of variables (see [KaP, Lep]). In the realization determined by $\hat{\mathscr{H}}$ the defining equations of the orbit of the associated Kac-Moody group through the highest weight vector are then differential equations in terms of the variables occurring in the corresponding vertex operators, see, e.g., $[\mathrm{KaW}]$. For the simplest choice of Heisenberg algebra, the principal one, one obtains the generalized Korteweg-de Vries hierarchies and it is mainly in this context that the Krichever map is studied (especially in connection with the Schottky problem [Mull, Sh]), but see also [Di].

The question we want to address in this paper is the significance of the choice of Heisenberg algebra (which plays such an important role in the representation theory) for the algebro-geometric picture.

First recall ([KaP, Lep]) that every partition $n$ of an integer $n$ determines a Heisenberg algebra $\mathscr{H}^{\underline{n}}$ in $\widehat{s l}(n, \mathbb{C})$, the non-twisted affine Kac-Moody algebra based on the loop group of $s l(n, \mathbb{C})$, and that every conjugacy class of Heisenberg algebras corresponds to one unique partition. The principal Heisenberg algebra $\hat{\mathscr{H}}^{\text {princ }}$ of $s l(n, \mathbb{C})$ corresponds to the partition of $n$ into a single part. Next recall that in the standard treatment of algebro-geometric solutions of generalized $\mathrm{KdV}$ hierarchies, corresponding to the principal Heisenberg $\hat{\mathscr{H}}^{\text {princ }}$, the geometric data for the Krichever map involves a Riemann surface $Y$ covering the Riemann sphere in such a way that inverse image of the North Pole ("infinity") is a single point of $Y$.

We will explain that this generalizes as follows: the number of points over infinity should correspond to the number of parts in the partition. More precisely, solutions of soliton hierachies, coming from other choices of Heisenbergs $\hat{\mathscr{H}}^{n}$, where $\underline{n}=\left(n_{1}, n_{2}, \ldots, n_{k}\right)$ is a partition of $n$ into $k$ parts, can be obtained from Riemann surfaces covering the Riemann sphere with $k$ points (with ramification index $n_{i}$ at the $i^{\text {th }}$ point) lying over the north pole.

In fact, we study a more general situation of algebraic curves $Y$ covering a fixed nonsingular and irreducible Riemann surface $X$. Any coherent torsion free sheaf on $Y$ will push forward to a vector bundle on $X$. The collection of vector bundles on $X$ of fixed rank together with a trivialization at some fixed point $p \in X$ forms an infinite dimensional Grassmannian $G r_{X}^{n}$ described in Sect. 2, (see also [PrS]). In this situation the Krichever map associates a point $W \in G r_{X}^{n}$ to any covering $f: Y \rightarrow X$, a coherent sheaf on $Y$ and data at $f^{-1}(p)$. This is described in detail in Sect. 5.

To invert the Krichever map and obtain the geometric data from the point $W \in G r_{X}^{n}$ we introduce in Sect. 3 the non-commutative stabilizer algebra $S_{W}$ of $W$. This is the subalgebra of elements $s$ of the loop algebra such that $s W \subset W$. We show that it may be thought of as a subspace (of codimension $2 n^{2} g$, with $g$ the genus of $X$ ) of the fiber of the cotangent bundle $T^{*} G r_{X}^{n}$, and that for the case of $X=\mathbf{P}^{1}$ it may be identified with this fiber. In a similar context cotangent bundles 
of moduli spaces have appeared earlier in Hitchin's work on Higgs bundles and spectral curves, see $[\mathrm{Hi}]$ (see also $[\mathrm{AHH}, \mathrm{B}])^{1}$

By projection from the Kac-Moody algebra to the loop algebra we obtain from every Heisenberg algebra $\hat{\mathscr{H}}^{n}$ a maximal Abelian subalgebra $\mathscr{H}^{n}$ of the loop algebra. We will refer also to this as a Heisenberg algebra (of type $\underline{n}$ ). The finite order part of the intersection $S_{W}^{n}=S_{W} \cap \mathscr{H}^{n}$ is a finitely generated commutative $\mathbb{C}$-algebra. So we can associate to every point of the infinite Grassmannian $\mathrm{Gr}_{X}^{n}$, $\phi(n)$ (the number of partitions of $n$ ) distinct commutative rings and we prove in Sects. 6 and 7 that the compactification of the spectrum of each of these rings is an algebraic curve $Y$ covering $X$ with the branching behaviour over $p \in X$ determined by the choice of partition. (In the best case the pullback divisor $f^{*}(p)$ is of the form $\sum n_{i} p_{i}, p_{i} \in Y$ if the $n_{i}$ are the parts of the partition. In general the situation can be considerably more complicated, see Theorem 7.5.1 for a more precise statement. We stress that for most $W$ the curve constructed by this method is just the original $X$, see Example 6.2.) We can also construct a coherent sheaf on $Y$ corresponding to the $S_{W}^{n}$ module $W$, as well as the other geometric data, so that we obtain in this way an inverse of the Krichever map, generalizing a procedure in [SeW], cf. also [Mum1]. (In this paper we study only the finite order parts of these stabilizer algebras so that our curves are algebraic. More generally one can study holomorphic stabilizer algebras but in that setting the geometric data may be more complicated, see Example 6.2.)

Now there are, again for every Heisenberg algebra, natural flows on the Grassmannian that preserve the curves and are called therefore isospectral. In Sect. 8 we briefly discuss the associated differential equations and their Hamiltonian structure. They turn out to be certain subsystems of the multicomponent KP hierarchy studied in, e.g., [UT, Di]. Other papers that consider the relation between vector bundles and soliton equations are [Mul2, PrW].

We conclude with some remarks in Sect. 9. Some of the results of this paper were announced in $[\mathrm{AB}]$.

\section{The Infinite Grassmannian $G r_{X}^{n}$}

2.0. Pressley and Segal introduced in [PrS] (Sect. 8.11) an infinite dimensional Grassmannian which can be identified with the space of holomorphic vector bundles over a Riemann surface $X$ together with an holomorphic trivialization in a disc around a fixed point $p \in X$. The Grassmannian consists of certain subspaces of the Hilbert space $L^{2}\left(S^{1}, \mathbb{C}^{n}\right)$, where $S^{1}$ is the boundary of the disk, given by the $L^{2}$-completion of the restriction of the holomorphic sections of the vector bundle in the complement of the disk to the boundary. There is a natural transitive action of the smooth loop group on this Grassmannian, so it is also an homogeneous space for this group. In this section we will describe a variant of their construction so as to study the space $G r_{X}^{n}$ of equivalence classes of pairs $(\mathscr{E}, t)$, where $\mathscr{E}$ is an algebraic vector bundle over $X$ and $t$ is an holomorphic trivialization of $\mathscr{E}$ at $p$. In this set-up there is no a priori connection between the radius of convergence of the local

\footnotetext{
${ }^{1}$ After completing this paper we received a preprint by Landi and Reina [LR] in which the holomorphic cotangent bundle to the Grassmannian is used to study the Hamiltonian structure of soliton equations. In that paper, only the principal Heisenberg algebra is used and also, the base curve is always $\mathbf{P}^{1}$
} 
holomorphic coordinate at $p$ and that of the local trivialization, cf. [ADKP]. Along the way we will also make some remarks about formal trivializations and the corresponding formal Grassmannian as in, for instance, [KNTY].

2.1. Let $X$ be an integral, smooth, projective, algebraic curve (over the complex numbers as always in this paper) and $p$ some point on $X$ fixed once and for all. In this sub-section we recall the definitions of algebraic and holomorphic local coordinates $\lambda^{-1}$ at $p$. (We use $\lambda^{-1}$ instead of just $\lambda$ so that our conventions match those of the usual infinite Grassmannian based on the Riemann sphere, where $p$ is considered to be the point at infinity.)

Let $\mathcal{O}_{X}$ be the (algebraic) structure sheaf of $X$ and let $\mathcal{O}_{X, p}$ be the ring of rational functions which are regular at $p$. This is a local ring with maximal ideal $\mathrm{m}_{p}$ given by functions which vanish at $p$. An algebraic local coordinate is a generator $\lambda^{-1}$ of $\mathrm{m}_{p}$. Notice that this defines an injective local $\mathbb{C}$-algebra homomorphism $l: \mathcal{O}_{X, p} \rightarrow \mathbb{C}\left[\lambda^{-1} \rrbracket\right.$ of the local ring to the ring of formal power series.

Recall [Ser] that an algebraic curve $\left(X, \mathcal{O}_{X}\right)$ (or more generally an algebraic variety) has canonically associated to it an analytic space $\left(X, \mathcal{O}_{X}^{\text {hol }}\right)$, where $\mathcal{O}_{X}^{\text {hol }}$ is the sheaf of germs of holomorphic functions on $X$. (So $X$ has now the complex, instead of the Zariski, topology.) To define an holomorphic local coordinate the ring $\mathcal{O}_{X, p}$ is replaced by $\mathcal{O}_{X, p}^{\text {hol }}$, the ring of germs of holomorphic functions at $p$ on $X$. This, too, is a local ring with maximal ideal $\mathrm{m}_{p}^{\text {hol }}$, the germs of holomorphic functions at $p$ which vanish at $p$. A generator $\lambda^{-1}$ of $\mathrm{m}_{p}^{\text {hol }}$ gives then an holomorphic local coordinate. As above, this defines an injective local homomorphism of $\mathbb{C}$-algebras $l: \mathcal{O}_{X, p}^{\text {hol }} \rightarrow \mathbb{C} \llbracket \lambda^{-1} \rrbracket$. (The image is $\mathbb{C}\left\{\lambda^{-1}\right\}$, the formal power series in $\lambda^{-1}$ which converge in some neighborhood of $\infty$.) Of course any algebraic local coordinate is also holomorphic. For both kinds of local coordinates we have by definition $l(1)=1$. We will denote the image $l\left(\mathcal{O}_{X, p}\right)$ by $A_{p}$, when $l$ is either algebraic or holomorphic.

From now on we will fix an holomorphic local coordinate $\lambda^{-1}$ (which might happen to be algebraic).

A local coordinate induces an injection:

$$
l: K_{X} \hookrightarrow L_{\lambda}=\mathbb{C}[\lambda] \oplus \lambda^{-1} \mathbb{C}\left\{\lambda^{-1}\right\} .
$$

Here $K_{X}$ is the function field of $X$, i.e., the quotient field of the integral domain $\mathcal{O}_{X, p}$, and $L_{\lambda}$ is the field of Laurent series (in $\lambda^{-1}$ ), the quotient field of the ring of convergent power series (in $\lambda^{-1}$ ). We will in the sequel not distinguish between $K_{X}$ and its image in $L_{\lambda}$.

For any point $q$ on $X$ the local ring $\mathcal{O}_{X, q}$ is a subring of $K_{X}$, so the map (2.1.1) gives a map $\mathcal{O}_{X, q} \subseteq K_{X} \subseteq L_{\lambda}$. Let us denote by $A_{q}=l\left(\mathcal{O}_{X, q}\right)$ the image in $L_{\lambda}$. Define

$$
H^{0}\left(X, \mathcal{O}_{X}(* p)\right)=\bigcup_{k=0}^{\infty} H^{0}\left(X, \mathcal{O}_{X}(k p)\right) .
$$

Then $H^{0}\left(X, \mathcal{O}_{X}(* p)\right)$ is the subring of $K_{X}$ consisting of the meromorphic functions on $X$ with only possible pole (of finite order) at $p . H^{0}\left(X, \mathcal{O}_{X}(* p)\right)$ is the (algebraic) coordinate ring of the affine curve $X-p$, in particular $H^{0}\left(X, \mathcal{O}_{X}(* p)\right)$ is a Dedekind domain. Since $H^{0}\left(X, \mathcal{O}_{X}(* p)\right)$ is the intersection of the local rings $\mathscr{O}_{X, q}$, $q \neq p$ we get an inclusion $H^{0}\left(X, \mathcal{O}_{X}(* p)\right) \subseteq L_{\lambda}$. We will denote the image by $A_{X}$, i.e.,

$$
A_{X}=\bigcap_{q \neq p} A_{q}=l\left(H^{0}\left(X, \mathcal{O}_{X}(* p)\right) .\right.
$$


Remark. The local ring $\mathcal{O}_{X, p}$ has the formal completion $\hat{\mathcal{O}}_{X, p}$; this is a local ring with maximal ideal $\hat{\mathrm{m}}_{p}$. A formal local coordinate at $p$ is given by $\hat{l}^{-1}\left(\lambda^{-1}\right)$, where $\hat{l}$ is any isomorphism of local $\mathbb{C}$-algebras $\hat{l}: \hat{\mathcal{O}}_{X, p} \simeq \mathbb{C} \llbracket \lambda^{-1} \rrbracket$; in particular the maximal ideal $\hat{\mathrm{m}}_{p}$ maps isomorphically to the ideal generated by $\lambda^{-1}$. We will denote the formal local coordinate simply by $\lambda^{-1}$. Using this we can inject the function field $K_{X}$ in $F_{\lambda}=\mathbb{C}[\lambda] \oplus \lambda^{-1} \mathbb{C}\left[\lambda^{-1}\right]$, the field of formal Laurent series. The field $L_{\lambda}$ is a subfield of $F_{\lambda}$ and this gives also $A_{X}$ as a subring of $F_{\lambda}$.

2.2. Examples. If $X$ has genus 0 , we take $\lambda$ to be the usual affine coordinate on $\mathbb{P}^{1}$, and $p$ the point at infinity. Then $\lambda^{-1}$ is an algebraic coordinate at $\infty$ and $A_{X}$ is the polynomial ring $\mathbb{C}[\lambda] \subset L_{\lambda}$.

If $X$ has genus 1 , i.e., $X$ is an elliptic curve, we use the Weierstrass $\wp$-function: for $z$ a local holomorphic coordinate around $p$ there is a unique meromorphic function $\wp(z)$ on $X$ which is holomorphic outside $p$ and has polar part $1 / z^{2}$ at $p$. Then, identifying $\wp(z)$ with its Laurent series in $L_{z^{-1}}$,

$$
A_{X}=\mathbb{C} \oplus \mathbb{C} \wp(z) \oplus \mathbb{C}_{\wp}(z) \oplus \mathbb{C} \wp(z)^{2} \oplus \cdots \subset L_{z^{-1}}
$$

If one prefers, one can introduce a new holomorphic local coordinate $\lambda^{-1}:=\wp(z)^{-1 / 2}$ around $p$. Then, using the differential equation relating the Weierstrass $\wp$-function and its derivative:

$$
\wp^{\prime}(z)^{2}=4 \wp(z)^{3}-g_{2} \wp(z)-g_{3},
$$

we get, defining $\mu:=\lambda^{3}\left(4-g_{2} \lambda^{-4}-g_{3} \lambda^{-6}\right)^{1 / 2}$, for the coordinate ring

$$
A_{X}=\mathbb{C}\left[\lambda^{2}, \mu\right] \subset L_{\lambda} .
$$

The inclusion $\mathbb{C}\left[\lambda^{2}\right] \subset A_{X}$ induces a very simple covering map $X \rightarrow \mathbf{P}^{1}$, showing that it is in general natural to choose a local coordinate $\lambda^{-1}$ such that a power of $\lambda$ has no poles outside $p$. We will see similar choices of local coordinates in Sect. 5. Note that here we might have started with $z$ being a local algebraic coordinate, but that if one wants $A_{X}$ to have the form (2.2.3) one ends up with $\lambda^{-1}$ being not algebraic, but holomorphic.

2.3. Now let $\mathscr{E}$ be the sheaf of algebraic sections of a rank $n$ algebraic vector bundle $E$ over $X$. We want to discuss trivializations of $\mathscr{E}$ at $p$. As with local coordinates this depends on whether we are working in the algebraic, holomorphic, or formal category. Recall that the stalk $\mathscr{E}_{p}$ is a free $\mathcal{O}_{X, p}$-module of rank $n$, and so is isomorphic to $\left(\mathcal{O}_{X, p}\right)^{n}$, the direct sum of $n$ copies of the stalk at $p$ of the structure sheaf. An algebraic trivialization of $\mathscr{E}$ at $p$ is a choice of isomorphism $i: \mathscr{E}_{p} \simeq\left(\mathcal{O}_{X, p}\right)^{n}$. If $\lambda^{-1}$ is a local holomorphic coordinate, inducing the injection $l: \mathcal{O}_{X, p} \rightarrow \mathbb{C}\left\{\lambda^{-1}\right\}$, we continue to call the composition

$$
t=l \circ i: \mathscr{E}_{p} \simeq\left(\mathcal{O}_{X, p}\right)^{n} \rightarrow\left(\mathbb{C}\left\{\lambda^{-1}\right\}\right)^{n},
$$

an algebraic trivialization, where $l$ acts componentwise.

Letting $\mathscr{E}^{\text {hol }}$ denote the sheaf of germs of holomorphic sections of $E$, we note that the stalk at $p, \mathscr{E}_{p}^{\text {hol }}$, is simply given by $\mathcal{O}_{X, p}^{\text {hol }} \otimes \mathscr{E}_{p}$ and that this is a free $\mathcal{O}_{X, p}^{\text {hol }}$ module of rank $n$. An holomorphic trivialization is a choice of isomorphism $i: \mathscr{E}_{p}^{\text {hol }} \leadsto\left(\mathcal{O}_{X}^{\text {hol }}\right)^{n}$. Again, if $\lambda^{-1}$ is a local holomorphic coordinate, giving the isomorphism $l:\left(\mathcal{O}_{X, p}^{\text {hol }}\right)^{n} \rightarrow\left(\mathbb{C}\left\{\lambda^{-1}\right\}\right)^{n}$, we continue to call the composition $t=l \circ i$ an holomorphic trivialization. 
Note that the basis picked out by the choice of the isomorphism $i$ corresponds under the trivialization $t$ to the standard basis $\left\{e_{i}\right\}_{i=0}^{n-1}$ of $\left(\mathbb{C}\left\{\lambda^{-1}\right\}\right)^{n}$, since $l(1)=1$, $e_{i}$ being the column vector with a 1 on the $i^{\text {th }}$ place as its only non-zero entry. (We have the, maybe deplorable, convention of indexing $n$-component vectors, matrices etc. by the integers $0,1, \ldots, n-1$.)

We will call two pairs $(\mathscr{E}, t)$ and $\left(\mathscr{E}^{\prime \prime}, t^{\prime}\right)$ isomorphic (notation: $\left.(\mathscr{E}, t) \sim\left(\mathscr{E}^{\prime}, t^{\prime}\right)\right)$ if there exists an isomorphism of $\mathcal{O}_{X}^{\text {hol }}$-modules $\phi: \mathscr{E}^{\text {hol }} \rightarrow \mathscr{E}^{\text {hol }}$ such that the induced map $t^{\prime} \circ \phi \circ t^{-1}:\left(\mathbb{C}\left\{\lambda^{-1}\right\}\right)^{n} \rightarrow\left(\mathbb{C}\left\{\lambda^{-1}\right\}\right)^{n}$ is the identity. In other words $\phi$ gives an isomorphism of the holomorphic vector bundles $E^{\text {hol }}$ and $E^{\prime \text { hol }}$ and maps one trivialization at $p$ to the other. We will denote the isomorphism class containing $(\mathscr{E}, t)$ by $[\mathscr{E}, t]$. We remark here that by the GAGA principle [Ser] $\phi$ actually comes from an isomorphism of the algebraic sheaves $\mathscr{E}$ and $\mathscr{E}$.

Definition 2.3.1. The set of equivalence classes $[\mathscr{E}, t]$, where $t$ is an holomorphic trivialization at $p$, is the Grassmannian, $G r_{X}^{n}$; the algebraic Grassmannian, $G r_{X}^{n \text {,alg }}$, is then the set of equivalence classes $[\mathscr{E}, t]$, where $t$ is an algebraic trivialization.

Note that our Grassmannians depend also on the choices of the point $p$ and of the local coordinate $\lambda^{-1}$, although, for simplicity's sake, we suppress this from the notation.

Remark. We can of course also define a formal Grassmannian $G r_{X}^{n \text {,form }}$ by taking equivalence classes of pairs $(\mathscr{E}, t)$, where $t$ is now a formal trivialization. To define formal trivializations introduce the formal stalk, $\hat{\mathscr{E}}_{p}$ of the bundle $E$ at $p$ by $\hat{\mathscr{E}}_{p}=\hat{\mathcal{O}}_{X, p} \otimes \mathscr{E}_{p}$. This is a free module of rank $n$ over $\hat{\mathcal{O}}_{X, p}$ and a choice of isomorphism $i: \hat{\mathscr{E}}_{p} \rightarrow\left(\hat{\mathcal{O}}_{X, p}\right)^{n}$ defines a formal trivialization $t=\hat{l} \circ i$, where $\hat{l}:\left(\hat{\mathcal{O}}_{X, p}\right)^{n} \rightarrow\left(\mathbb{C} \llbracket \lambda^{-1} \rrbracket\right)^{n}$ is given by the formal local coordinate.

2.4. In this subsection we describe $G r_{X}^{n}$ as a collection of subspaces of the space of $n$-component Laurent series $L_{\lambda}^{n}$. We first recall the $X$-lattice approach to vector bundles (cf. [H]). Let $V$ be an $n$ dimensional vector space over the function field $K_{X}$ of $X$ and let a family $\left\{M_{q} \subset V\right\}_{q \in X}$ be given where $M_{q}$ is a free rank $n$ module over the local ring $\mathcal{O}_{X, q}$. Assume furthermore that for almost all $q \in X$, for some fixed basis $\varepsilon_{i}$ of $V, M_{q}=\oplus \mathcal{O}_{X, q} \varepsilon_{i}$. Then the family $\left\{M_{q}\right\}$ is (essentially) what is called an $X$-lattice in $[\mathrm{H}]$. Their study leads to the adèlic description of the moduli space of vector bundles over $X$, cf. also [AtB].

Let $\mathscr{E}_{0}=K_{X} \otimes_{\mathcal{O}_{X}} \mathscr{E}$ be the space of meromorphic sections of our rank $n$ vector bundle $E$. This is an $n$ dimensional vector space over $K_{X}$ and there is for every $q$ in $X$ an injection $i_{q}: \mathscr{E}_{q} \rightarrow \mathscr{E}_{0}$ from the stalk at $q$ to the "generic stalk" $\mathscr{E}_{0}$. The image of $i_{q}$ will be still denoted by $\mathscr{E}_{q}$. Then the collection $\left\{\mathscr{E}_{q}\right\}$ in $\mathscr{E}_{0}$ is an $X$-lattice. Conversely every $X$-lattice $\left\{M_{q}\right\}$ determines a locally free sheaf $\mathscr{E}$ by defining $\mathscr{E}(U)=\bigcap_{q \in U} M_{q}$ for all open $U$ in $X$. Isomorphic locally free sheaves $\mathscr{E}, \mathscr{E}$ give rise to equivalent $X$-lattices, i.e., families $\left\{\mathscr{E}_{q}\right\},\left\{\mathscr{E}_{q}^{\prime}\right\}$ with $\mathscr{E}_{q}=\gamma \mathscr{E}_{q}^{\prime}$ for $\gamma \in G l\left(n, K_{X}\right)$, and vice versa.

Now return to the situation where we fix an holomorphic trivialization $t$ of $\mathscr{E}$ at p. We can identify the space of meromorphic sections of $\mathscr{E}$ with $K_{X} \otimes \mathscr{E}_{p}$. Any element $e$ of $\mathscr{E}_{q}$ extends to a meromorphic section of $E$, so determines, by taking the germ at $p$, an element $i_{q}(e)$ of $K_{X} \otimes \mathscr{E}_{p}$. The holomorphic trivialization $t$ induces (using also (2.1.1)) an homomorphism:

$$
t: K_{X} \otimes \mathscr{E}_{p} \rightarrow L_{\lambda}^{n}
$$


We will still denote by $\mathscr{E}_{q}$ the image in $L_{\lambda}^{n} \equiv\left(L_{\lambda}\right)^{n}$. In this way we obtain an $X$-lattice $\left\{\mathscr{E}_{q}\right\}$ in $t\left(K_{X} \otimes \mathscr{E}_{p}\right)$ inside $L_{\lambda}$.

Lemma 2.4.1. Isomorphic pairs $(\mathscr{E}, t)$ give identical families $\left\{\mathscr{E}_{q}\right\}$ in $L_{\lambda}^{n}$.

Proof. Assume $(\mathscr{E}, t) \sim\left(\mathscr{E}^{\prime \prime}, t^{\prime}\right)$ and let $\phi$ denote the isomorphism. Then $\phi$ gives isomorphisms $\phi_{q}: \mathscr{E}_{q} \rightarrow \mathscr{E}_{q}^{\prime}$ and $\phi_{p}: \mathscr{E}_{p} \rightarrow \mathscr{E}_{p}^{\prime}$ and we know that $t=t^{\prime} \circ \phi_{p}$. Let $i_{q}$ denote the map $\mathscr{E}_{q} \rightarrow K_{X} \otimes \mathscr{E}_{p}$ and $i_{q}^{\prime}$ the corresponding map on $\mathscr{E}_{q}^{\prime}$. Then the following diagram commutes:

$$
\begin{aligned}
& \mathscr{E}_{q} \stackrel{i_{q}}{\longrightarrow} K_{X} \otimes \mathscr{E}_{p} \stackrel{t}{\longrightarrow} L_{\lambda} \\
& \phi_{q} \downarrow \quad \phi_{p} \downarrow \quad \| \text {. } \\
& \mathscr{E}_{q}^{\prime} \underset{i_{q}^{\prime}}{\longrightarrow} K_{X} \otimes \mathscr{E}_{p}^{\prime} \underset{t^{\prime}}{\longrightarrow} L_{\lambda}
\end{aligned}
$$

Since we wished to show that $t \circ i_{q}=t^{\prime} \circ i_{q}^{\prime} \circ \phi_{q}$, this proves the Lemma.

The vector bundle analogue of (2.1.2) is the following:

$$
H^{0}(X, \mathscr{E}(* p)):=\bigcup_{k \geqq 0} H^{0}(X, \mathscr{E}(k p)) .
$$

This is the space of meromorphic sections of $E$ with possible poles at $p$, and it is the intersection (in $K_{X} \otimes \mathscr{E}_{p}$ ) of the $\mathscr{E}_{q}, q \neq p$. So, using the trivialization as described above, we get a subspace in $L_{\lambda}^{n}$ :

$$
W=W(\mathscr{E}, t)=\bigcap_{p \neq q} \mathscr{E}_{q} \subset L_{\lambda}^{n},
$$

which depends only on the isomorphism class $[\mathscr{E}, t]$, by the commutative diagram 2.4.2. This is a finitely generated projective module of rank $n$ over $A_{X}$, the coordinate ring of $X-p$. In general this module is not free, however it has the following property: the $K_{X}$-vector space $V=K_{X} \otimes_{A_{X}} W=t\left(K_{X} \otimes \mathscr{E}_{p}\right) \subset L_{\lambda}^{n}$ has a basis $\left\{\varepsilon_{i}=\gamma e_{i}\right\}$ with $\gamma \in G l\left(n, \mathbb{C}\left\{\lambda^{-1}\right\}\right)$. Indeed any (algebraic) basis of $\mathscr{E}_{p}$ gives a basis of $V$ and will be related to the trivializing basis of the holomorphic stalk, $\left\{e_{i}\right\}$, by an element of $G l\left(n, \mathbb{C}\left\{\lambda^{-1}\right\}\right)$. The $W$ 's arising from algebraic trivializations are then characterized by the fact that $V=K_{X} \otimes_{A_{X}} W$ has a basis related to $\left\{e_{i}\right\}$ by an element of $G l\left(n, A_{p}\right)$, so that in this case we could take in fact $\left\{e_{i}\right\}$ as basis for $V$.

Conversely, let $W$ be a finitely generated projective $A_{X}$-module of rank $n$ in $L_{\lambda}^{n}$ such that $V=K_{X} \otimes_{A_{X}} W$ has some basis $\left\{\varepsilon_{i}\right\}$ related to the standard basis by an element of $G l\left(n, \mathbb{C}\left\{\lambda^{-1}\right\}\right)$. Then we have for each $q \in X_{p}$ an $A_{q}=\mathcal{O}_{X, q}$-module $\mathscr{E}_{q}=A_{q} \otimes W$ of rank $n$, which is free because $W$ is projective. Automatically the $\mathscr{E}_{q}$ are of the form $\oplus A_{q} \varepsilon_{i}$ for almost all $q \in X-p$. Indeed any basis $\left\{\bar{\varepsilon}_{i}\right\}$ of $\mathscr{E}_{\bar{q}}$ for some $\bar{q} \in X-p$ will be a basis also for almost all $\mathscr{E}_{q}$ and $\left\{\bar{\varepsilon}_{i}\right\}$ is related to $\left\{\varepsilon_{i}\right\}$ by an element of $G l\left(n, K_{X}\right)$. To complete the collection $\left\{\mathscr{E}_{q}\right\}_{q \in X-p}$ to an $X$-lattice in $V$ (in order to obtain a locally free sheaf) we just have to specify $\mathscr{E}_{p}$. Any free $A_{p}$ module in $V$ will do, so we can take $\mathscr{E}_{p}=\oplus A_{q} \varepsilon_{i}$. Any other choice of basis $\left\{\varepsilon_{i}^{\prime}\right\}$ of $V$, with $\varepsilon_{i}^{\prime}=\gamma^{\prime} e_{i}, \gamma^{\prime} \in G l\left(n, \mathbb{C}\left\{\lambda^{-1}\right\}\right)$, will be related by an element of $G l\left(n, K_{X}\right) \cap G l\left(n, \mathbb{C}\left\{\lambda^{-1}\right\}\right)=G l\left(n, A_{p}\right)$ to $\left\{\varepsilon_{i}\right\}$. This does not change $\mathscr{E}_{p}$ and so gives the same $X$-lattice and the same locally free sheaf $\mathscr{E}$. This sheaf has a distinguished trivialization $t_{i}=\gamma^{-1} \varepsilon_{i}=\gamma^{\prime-1} \varepsilon_{i}^{\prime}$. Note that the construction depends on the choice of basis $\left\{e_{i}\right\}$ in $L_{\lambda}^{n}$ and that we are free to perform $G l\left(n, K_{X}\right)$ transformations on it. Taking this into account we find that we have proven 
Proposition 2.4.2. $G r_{X}^{n}$ is in one to one correspondence with the collection of rank $n$ projective $A_{X}$-modules $W$ in $L_{\lambda}^{n}$ such that $V=K_{X} \otimes_{A_{X}} W$ has a basis $\left\{\varepsilon_{i}=g e_{i}\right\}$, where the $g$ belongs to $G l\left(n, \mathbb{C}\left\{\lambda^{-1}\right\}\right)$.

The algebraic Grassmannian is distinguished by the fact that the standard basis $\left\{e_{i}\right\}$ gives a basis for $V$, or in other words that the element $g$ connecting the standard basis $\left\{e_{i}\right\}$ and $\left\{\varepsilon_{i}\right\}$ belongs to the subgroup $G l\left(n, A_{p}\right)$.

Remark. The formal Grassmannian is similarly equivalent to the finitely generated rank $n$ projective $A_{X}$-modules $W$ in $F_{\lambda}^{n}$, such that $V=K_{X} \otimes_{A_{X}} W$ has a basis $\left\{\varepsilon_{i}=g e_{i}, g \in G l\left(n, \llbracket \lambda^{-1} \rrbracket\right)\right\}$ that is also a basis for almost all localizations.

2.5. Let us consider the special case where $X=\mathbf{P}^{1}$ and $\lambda^{-1}$ is the standard algebraic coordinates at $\infty$. Then $A_{X}$ is the principal ideal domain $\mathbb{C}[\lambda]$ and all locally free $A_{X}$ modules $W \subset L_{\lambda}$ coming from an element of $G r_{P^{1}}^{n}$ are in this case free as $A_{X}$-modules. Geometrically this reflects the fact that all algebraic vector bundles over $\mathbf{P}^{1}-\infty$ are algebraically trivializable. So we have a basis $\left\{w_{i}\right\}$ for $W$ which gives, by juxtaposition, an element $g$ of $G l\left(n, L_{\lambda}\right)$, i.e., the columns of $g$ are the $w_{i}$ 's. (We can think of $G l\left(n, L_{\lambda}\right)$ as the group of real analytic maps from an infinitesimal circle around $p$ with only possibly a finite order pole at $p$ in the interior of the circle.) This shows that for $X=\mathbf{P}^{1}$ the elements of the Grassmannian lie on the orbit of $\operatorname{Gl}\left(n, L_{\lambda}\right)$ through the distinguished subspace

$$
H_{X}^{(n, \mathrm{alg})}=\bigoplus_{i=0}^{n-1} A_{X} e_{i} \subset L_{\lambda}^{n},
$$

corresponding to the trivial bundle $\mathcal{O}_{\mathbf{P}^{1}}^{n}$ with its natural trivialization at $\infty$, since $W=g H_{X}^{(n, \text { alg })}$.

Conversely for any Riemann surface an element of the $G l\left(n, L_{\lambda}\right)$ orbit through $H_{X}^{(n, \text { alg })}$ lies in the Grassmannian $G r_{X}^{n}$. This follows from the following proposition:

Proposition 2.5.1. Let $g \in G l\left(n, L_{\lambda}\right)$, then

$$
g=g_{K} g_{-}, \text {with } g_{K} \in G l\left(n, K_{X}\right), \quad g_{-} \in G l\left(n, \mathbb{C}\left\{\lambda^{-1}\right\}\right) .
$$

Proof. Let $\sigma^{-1}$ be an algebraic local coordinate at $p$. Then there is a unique isomorphism $\phi: L_{\lambda} \rightarrow L_{\sigma}$ that restricts to an isomorphism $\mathbb{C}\left\{\lambda^{-1}\right\} \rightarrow \mathbb{C}\left\{\sigma^{-1}\right\}$. We extend $\phi$ by componentwise action to matrices. Then by the Birkhoff factorization (cf. [PrS, Theorem 8.2.1]) we have for $\tilde{g}=\phi(g)$ a decomposition $\tilde{g}=\tilde{g}_{+} \cdot D \cdot \tilde{g}_{-}$, with $\tilde{g}_{+}$polynomial in $\sigma, \quad D=\operatorname{diag}\left(\sigma^{a_{1}}, \sigma^{a_{2}}, \ldots, \sigma^{a_{n}}\right), \quad a_{i} \in \mathbb{Z} \quad$ and $\tilde{g}_{-} \in G l\left(n, \mathbb{C}\left\{\sigma^{-1}\right\}\right)$. Then, clearly, $\tilde{g}_{+} \cdot D \in G l\left(n, K_{X}\right)$ and applying $\phi^{-1}$ proves the proposition.

So if $g \in G l\left(n, L_{\lambda}\right)$ then $W=g H_{X}^{(n, \text { alg })}=\bigoplus_{i=0}^{n-1} A_{X} g e_{i}=\bigoplus_{i=0}^{n-1} A_{X} g_{K} \varepsilon_{i}$, with $\left\{\varepsilon_{i}=g_{-} e_{i}\right\}$. Then $V=K_{X} \otimes W$ has the basis required by Proposition 2.4 .2 and the free, rank $n, A_{X}$-module $W$ belongs to the Grassmannian. If $g$ - happens to belong to $G l\left(n, A_{p}\right)$ we get in this manner an element of the algebraic Grassmannian.

Since this $W$ is free as an $A_{X}$ module, we see that for general $X$ the orbit of $G l\left(n, L_{\lambda}\right)$ through the element (2.5.1) will give us the part of the Grassmannian corresponding to bundles $\mathscr{E}$ such that $\mathscr{E}$ is algebraically trivializable over $X-p$. For $X=\mathbf{P}^{1}$ this gives the whole Grassmannian.

To describe the Grassmannian for an arbitrary curve $X$ as an homogeneous space, we will in the next subsection change the framework to allow the action of 
matrices with infinite order poles at $p$, essentially by tensoring everything in sight by the appropriate ring of holomorphic functions. Another approach would be to use the $X$-lattices of $A_{p}$ modules $\mathscr{E}_{p}$ included in the $n$-component Laurent series $L_{\lambda}^{n}$ (or in the $n$-component formal Laurent series $F_{\lambda}^{n}$ in the formal category) briefly mentioned in Sect. 2.4. This would exhibit our Grassmannians as homogeneous spaces of adèlic groups, which seems to be more complicated than the holomorphic loop group setting that we are going to use. However if one is interested in these curves in arbitrary characteristic (for instance in the context of string theory, see for example $[\mathrm{KaSU}])$, the adèlic setting seems appropriate.

2.6. We have noted in the previous subsection that for $X=\mathbf{P}^{1}$ an element $W$ of the Grassmannian is free as an $A_{X}$-module. This is not the case, of course, for an arbitrary algebraic curve $X$. However, as is well known, every holomorphic bundle on $X-p$, now thought of as a complex manifold, is trivial, since $X-p$ is a Stein manifold. So we can consider instead of $W$ the tensor product $W^{\text {hol }}:=A_{X}^{\text {hol }} \otimes_{A_{X}} W$, which is then free of rank $n$ over $A_{X}^{\text {hol }}$, where $A_{X}^{\text {hol }}$ is the ring of holomorphic functions on $X-p$, usually denoted $\mathcal{O}(X-p)$. If $W$ corresponds to the equivalence class of a pair $(\mathscr{E}, t)$, then $W^{\text {hol }}$ is the space $H^{0}\left(X-p, \mathscr{E}{ }^{\text {hol }}\right)$ of holomorphic sections of $\mathscr{E}^{\text {hol }}$ over $X-p$. Now $A_{X}^{\text {hol }}$ is not contained in $L_{\lambda}$, since there are in $A_{X}^{\text {hol }}$ functions with essential singularities at $p$. This leads us to look for a space in which both $L_{\lambda}$ and $A_{X}^{\text {hol }}$ are contained. This will also allow us later on to consider "time flows" of points of the Grassmannians with exponential singularities.

Let $\lambda^{-1}$ be a local holomorphic coordinate at $p$. We can think of $\lambda^{-1}$ as an holomorphic function from a neighborhood of $p \in X$ to a neighborhood of the origin in $\mathbb{C}$ and we can consider for small $\varepsilon$ the disks $D_{\varepsilon}$ of radius $\varepsilon$ around $p \in X$, using the holomorphic local coordinate. Let $\dot{D}_{\varepsilon}$ be this disk with $p$ deleted. Define then (cf. [ADKP]):

$$
H=\lim _{\varepsilon \rightarrow 0} \mathcal{O}_{X}^{\text {hol }}\left(\dot{D_{\varepsilon}}\right),
$$

the space of germs of functions holomorphic in a deleted neighborhood of $p$. We can think of $H$ as consisting of certain functions with, in general, expansions in $\lambda$ infinite in both directions, in which case we will write $H=H_{\lambda}$. In the sequel we will refer to functions (or more generally vectors or matrices) having a finite number of positive powers in $\lambda$ in their expansions as being finite order. For instance the subfield of Laurent series $L_{\lambda}$ of the field of formal Laurent series $F_{\lambda}$ makes up the finite order elements in $H_{\lambda}$ (and hence, in particular $K_{X}$ is made up of finite order elements). However $A_{X}^{\text {hol }}$, which contains functions with essential singularities at $p$, is also included in $H_{\lambda}$, but a general element of $F_{\lambda}$ does not belong to $H_{\lambda}$.

The group $G l\left(n, H_{\lambda}\right)$ acts on $H_{\lambda}^{n}=\mathbb{C}^{n} \otimes_{\mathbb{C}} H_{\lambda}$. Put

$$
H_{X}^{(n, \text { hol })}=\bigoplus_{i=0}^{n-1} A_{X}^{\text {hol }} e_{i} \subset H_{\lambda}^{n} .
$$

Consider an element $W$ of the Grassmannian $G r_{X}^{n}$. Then $W^{\text {hol }}=A_{X}^{\text {hol }} \otimes W$ is a subspace of $H_{\lambda}^{n}$.

Lemma 2.6.1. The map

$$
W \mapsto W^{\text {hol }}=A_{X}^{\text {hol }} \otimes W
$$

from elements $W \in G r_{X}^{n}$ to subspaces of $H_{\lambda}^{n}$ is injective. 
Proof. Let $W^{\prime}$ be the finite order part of $W^{\text {hol }}$ so that we have an exact sequence of $A_{X}$-modules:

$$
0 \rightarrow W \rightarrow W^{\prime} \rightarrow W^{\prime} / W \rightarrow 0
$$

Since $A_{X}^{\text {hol }}$ is flat over $A_{X}$ we get, by tensoring, another exact sequence:

$$
0 \rightarrow A_{X}^{\text {hol }} \otimes W \rightarrow A_{X}^{\text {hol }} \otimes W^{\prime} \rightarrow A_{X}^{\text {hol }} \otimes\left(W^{\prime} / W\right) \rightarrow 0 .
$$

Now $W^{\text {hol }}=A_{X}^{\text {hol }} \otimes W^{\prime}=A_{X}^{\text {hol }} \otimes W$ so we get $A_{X}^{\text {hol }} \otimes\left(W^{\prime} / W\right)=0$. On the other hand we can construct (see 2.4) from $W$ and $W^{\prime}$ coherent sheaves $\mathscr{E}$ and $\mathscr{E}$ on $X$ by gluing in the stalk $\mathscr{E}_{p}=\mathscr{E}_{p}^{\prime}=\bigoplus A_{p} \varepsilon_{i}$ at $p$, where $\varepsilon_{i}$ is the basis of $V=W_{X} \otimes W$ that exists by Proposition 2.4.2. Then $\mathscr{E}^{\prime} / \mathscr{E}$ is an algebraic coherent sheaf on the projective curve $X$ and $\mathcal{O}_{X}^{\text {hol }} \otimes\left(\mathscr{E}^{\prime} / \mathscr{E}\right)$ the corresponding analytic sheaf. But by the sequence 2.6 .5 and because $\mathscr{E}_{p}=\mathscr{E}_{p}^{\prime}$ we see that in fact $\mathcal{O}_{X}^{\text {hol }} \otimes\left(\mathscr{E}^{\prime} / \mathscr{E}\right)=0$, so, by GAGA, also $\mathscr{E}^{\prime} / \mathscr{E}=0$ and hence $W=W^{\prime}$. So the finite order part of $W^{\text {hol }}$ is $W$ and $W^{\text {hol }}$ determines $W$ uniquely.

As we said before $W^{\text {hol }}$ is free of rank $n$ over $A_{X}^{\text {hol }}$, so we have a basis $\left\{w_{i}\right\}_{i=1}^{n}$ of $W^{\text {hol }}$. Then $W^{\text {hol }}=g H_{X}^{(n, \text { hol })}$, for $g \in G l\left(n, H_{\lambda}\right)$ given by the juxtaposition of the basis $\left\{w_{i}\right\}_{i=1}^{n}$. The stabilizer of $H_{X}^{(n, . \text { hol })}$ in $G l\left(n, H_{\lambda}\right)$ is the subgroup $G l\left(n, A_{X}^{\text {hol }}\right)$ so we find that the (holomorphic) Grassmannian is contained in the homogeneous space:

$$
G l\left(n, H_{\lambda}\right) / G l\left(n, A_{X}^{\text {hol }}\right) .
$$

In fact,

\section{Proposition 2.6.2.}

$$
G r_{X}^{n}=G l\left(n, H_{\lambda}\right) / G l\left(n, A_{X}^{\mathrm{hol}}\right) .
$$

Proof. It remains to show that if $\tilde{W}=g H_{X}^{(n, \text { hol })}$, for $g \in G l\left(n, H_{\lambda}\right)$, then $\tilde{W}=W^{\text {hol }}$ for some $W$ in $G r_{X}^{n}$. Since $g$ gives an holomorphic matrix valued function on a deleted neighborhood $\dot{D}_{\varepsilon}$ of $p$ it gives a transition function from $D_{\varepsilon}$ to $X-p$, and thus an holomorphic vector bundle on $X$ with trivializations on $D_{\varepsilon}$ and $X-p$. The sections of this bundle over $X-p$ in the trivialization over $D_{\varepsilon}$ give back $\tilde{W}$. By the GAGA principle, every holomorphic vector bundle on $X$ is indeed algebraic and thus $\tilde{W}=W^{\text {hol }}$ for this algebraic bundle with holomorphic trivialization at $p$.

Remark. We have seen in Sect. 2.5 that $W \in G r_{X}^{n}$ is free iff $W=g H_{X}^{(n, \text { alg })}$ for some $g \in G l\left(n, L_{\lambda}\right)$. So in this case we also have $W^{\text {hol }}=g H_{X}^{(n, \text { hol })}$ for the same $g$. The converse is also true: if $W^{\text {hol }}=g H_{X}^{(n, \text { hol })}$ for a finite order $g$ then $W$ is free, as easily follows from Lemma 2.6.1.

If $\left\{w_{i}\right\}_{i=1}^{n}$ is a set of linearly independent elements of $W$ (over $A_{X}$ ) then the images of these elements in $W^{\text {hol }}=A_{X}^{\text {hol }} \otimes W$ are independent over $A_{X}^{\text {hol }}$. However, since $A_{X}^{\text {hol }}$ is not a field, they may not span. If one takes the element $g \in G l\left(n, L_{\lambda}\right)$ obtained by juxtaposition of the vectors $w_{i}$ then the vector bundle $\overline{\mathscr{E}}$ obtained by this transition matrix will be algebraically trivializable over $X-p$. Its algebraic sections over $X-p$ will be given by $g H_{X}^{(n, \text { alg })}$ which is in general just a subset of the finite order part $W$ of $W^{\text {hol }}$ : there will be elements of $W$ not obtainable by $A_{X}$-linear combinations of the finite order set $w_{i}=g \cdot e_{i}$, except in case that the $w_{i}$ form a basis for $W$ over $A_{X}$ to begin with. So the algebraic and holomorphic bundles $\bar{E}, \overline{\mathscr{E}}^{\text {hol }}$ are just subbundles of $\mathscr{E}$ and $\mathscr{E}^{\text {hol }}$, in general.

2.7. Any Riemann surface $X$ admits a covering map $f: X \rightarrow \mathbf{P}^{1}$ of degree $k$, for all sufficiently large $k$, so in a sense all Grassmannians $G r_{X}^{n}$ based on $X$ are included in 
the Grassmannian $G r_{\mathbf{P}^{1}}^{n k}$ (in many different ways). More concretely, if we choose for instance, as in Sect. 2.2, the local coordinate $\lambda$ on $X$ at $p$ in such a way that $A_{X}=\mathbb{C} \oplus \mathbb{C} \lambda^{k} \oplus \ldots$ we can define $z=\lambda^{k}$ and think of $z$ as the standard algebraic coordinate on $\mathbf{P}^{1}$. Then $A_{X}$ is free over $\mathbb{C}[z]$ of rank $k$ and hence $W \in G r_{X}^{n}$ is free of rank $n k$ over $\mathbb{C}[z]$. We can identify in an obvious way $L_{\lambda}^{n}$ with $L_{z}^{k n}$ (see Sect. 4.2) and using this we see that $W$ is an element of $G r_{\mathbf{P}^{1}}^{n k}$ corresponding to the class $\left[f_{*} \mathscr{E}, f_{*} t\right]$, if $W$ corresponds to the bundle $\mathscr{E}$. Here $f_{*} t$ is the trivialization of the push forward bundle $f_{*} \mathscr{E}$ determined by the basis $\left\{z^{j} t^{-1}\left(e_{i}\right)\right\}_{i, j=0}^{i=n-1, j=k-1}$ of the $\hat{\mathcal{O}}_{\mathbf{P}^{1}, \infty}$ module $\left(f_{*} \widehat{\mathscr{E}}\right)_{\infty} ;$ this will be explained in more detail in Sect. 5 .

One can also map $G r_{X}^{n}$ into an universal Grassmannian $G r$ of subspaces $W$ of $H_{z}$ (see 2.6) that have a Fredholm projection $W \rightarrow H_{z,+}$, where $H_{z,+}$ is the subspace of $H_{z}$ consisting of holomorphic functions on $\mathbf{P}^{1}-\infty$ (here we think of $z$ as a coordinate on $\mathbf{P}^{1}$ ). (This is the definition in [ADKP], see also [SeW, PrS].) This works as follows. First note that $A_{X} \subset L_{\lambda}$ has a projection to $\mathbb{C}[\lambda]$ with zero kernel and cokernel of dimension $g$. Then $H_{X}^{(n, a l g)}$, and thus also $H_{X}^{(n, \text { hol })}=A_{X}^{\text {hol }} \otimes H_{X}^{(n, \text { alg })}$, has a projection to $\mathbb{C}[\lambda]$ (respectively $H_{\lambda,+}^{n}$ ) with zero kernel and cokernel of dimension $n g$. Finally, we use the isomorphism $H_{\lambda}^{n} \rightarrow H_{z}$ given by $\lambda^{k} e_{i} \mapsto z^{n k+i}$ to get a subspace $W(X, n) \subset H_{z}$ which belongs to $G r$. Now, $G r$ is an homogeneous space of the group $A_{\infty}$ consisting of invertible operators on $H_{z}$ whose diagonal blocks with respect to the decomposition $H_{z}=H_{z,+} \oplus H_{z,-}$ are Fredholm; here $H_{z,-}$ is the ring of convergent power series in $z^{-1}$. The action of $G l\left(n, H_{\lambda}\right)$ on $H_{\lambda}^{n}$ then translates to an action of a subgroup $\widetilde{G} l\left(n, H_{\lambda}\right)$ of $A_{\infty}$ on $H_{z}$. Thus we see that $G r_{X}^{n}=G l\left(n, H_{\lambda}\right) H_{\lambda}^{n}$, hol corresponds to the orbit $\tilde{G l}\left(n, H_{\lambda}\right) W(X, n)$ contained in the universal Grassmannian $G r$. In fact the points of $G r_{X}^{n}$ considered in $G r$ are characterized by the fact that they are stable under the action of the image of $A_{X}^{\text {hol }}$ thought of as a subring of $H_{z}$ using the map $\lambda \mapsto z^{n}$ (cf. [PrS]). (Note that for every $n$ we get a different subring in $H_{z}$ isomorphic to $A_{X}^{\text {hol }}$.) The fact that $W(X, n)$ belongs to $G r$ implies that there exists an infinite matrix $\gamma(X, n) \in A_{\infty}$ such that

$$
W(X, n)=\gamma(X, n) H_{z,+} .
$$

In general $\gamma(X, n)$ however will not belong to the image of the loop group in the group of infinite matrices.

\section{The Cotangent Bundle to the Grassmannian}

3.1. In this section we study the cotangent bundle to the Grassmannian $G r_{X}^{n}$ of vector bundles on $X$ and we will show that the fiber of the cotangent bundle at a point $W$ contains as a finite codimensional subspace the stabilizer algebra $S_{W}^{\text {hol }}$ of $W^{\text {hol }}$ : the linear transformations of $H^{n}$ that map $W^{\text {hol }}$ onto itself. In the sequel, commutative subalgebras of the finite order part of $S_{W}^{\text {hol }}$ will be used to construct covering spaces for $X$.

3.2. We recall here some well known facts about symplectic reduction for homogeneous spaces, see for more details e.g., Guillemin, Sternberg [GS1]. Let $G$ be a Lie group, $g$ its Lie algebra and denote by $L_{g}, R_{g}$ the action of $g \in G$ on $G$ from the left and the right respectively. These actions lift to symplectic actions on the cotangent bundle $T^{*} G$ with moment maps $J_{L}, J_{R}: T^{*} G \rightarrow \mathrm{g}^{*}$ given by $J_{L}\left(\mu_{g}\right)=R_{g}^{*} \mu_{g}, J_{R}\left(\mu_{g}\right)=-L_{g}^{*} \mu_{g}$ for all $\mu_{g} \in T_{g}^{*} G$. We trivialize $T^{*} G$ by right identification: $\mu_{g} \in T_{g}^{*} G$ is identified with the pair $(g, \mu) \in G \times \mathrm{g}^{*}$, such that 
$\langle\mu, X\rangle=\left\langle\mu_{g}, R_{g *} X\right\rangle, X \in \mathfrak{g}$. Then in these coordinates

$$
\begin{aligned}
\mathfrak{g}^{*} \stackrel{J_{L}}{\longleftarrow} G \times \mathrm{g}^{*} \stackrel{J_{R}}{\longrightarrow} \mathrm{g}^{*} \\
\mu \stackrel{\longleftarrow}{\longleftarrow}(g, \mu) \longrightarrow-\operatorname{Ad}_{g^{-1}}^{*}(\mu),
\end{aligned}
$$

where $\mathrm{Ad}_{g}^{*}$ denotes the coadjoint action of $g$ on $\mathrm{g}^{*}$, i.e., $\mathrm{Ad}_{g}^{*}$ is the dual map to $\operatorname{Ad}_{g-1}$.

Now let $K$ be a Lie subgroup of $G$ and $f$ the corresponding subalgebra of $g$. The right action of $G$ on $T^{*} G$ restricts to a right action of $K$ with moment map $J_{R, K}: T^{*} G \rightarrow \mathfrak{f}^{*}$. In fact $J_{R, K}=\pi_{\mathfrak{f}^{*}} \circ J_{R}$, where $\pi_{\mathfrak{f}^{*}}$ is the restriction of linear forms on $\mathfrak{g}$ to $\mathrm{f}$.

Now consider the Marsden-Weinstein reduction $J_{R, K}^{-1}(0) / K$ at $0 \in \mathfrak{f}^{*}$ of $T^{*} G$ under the $K$-action. The inverse image of zero under $\pi_{\mathfrak{f} *}$ is isomorphic to $(\mathfrak{g} / \mathfrak{f})^{*}$. Let us choose a complement of $\mathfrak{l}$ in $\mathfrak{g}: \mathfrak{g}=\mathfrak{L} \oplus \mathfrak{I}$. Here $\mathfrak{L}$ is just a vector subspace, not in general a subalgebra. Then $(\mathfrak{g} / \mathfrak{f})^{*} \simeq \mathfrak{2}^{*}$ and

$$
J_{R, K}^{-1}(0)=\left\{(g, \mu) \in G \times \mathfrak{g}^{*} \mid \operatorname{Ad}_{g^{-1}}^{*}(\mu) \in \mathfrak{Q}^{*}\right\} .
$$

Now the right action of $K$ on $T^{*} G$ in the right trivialization is just $(g, \mu) \mapsto(g k, \mu)$, hence the Marsden-Weinstein reduction of $T^{*} G$ by this action in this trivialization is a bundle over the quotient $G / K$, with fiber at the point $g K$ given by the space $\operatorname{Ad}_{g}^{*}\left(\mathfrak{Q}^{*}\right)$. If we choose on $\mathfrak{g}$ an Ad-invariant nondegenerate bilinear form $\langle$,$\rangle :$ $\mathfrak{g} \times \mathfrak{g} \rightarrow \mathbb{C}$ we can identify $\mathfrak{g}^{*}$ with $\mathfrak{g}$ and under this identification $\mathfrak{L}^{*}$ gets identified with the perpendicular $\mathfrak{f}^{\perp}=\{x \in \mathfrak{g} \mid\langle x, y\rangle, \forall y \in \mathfrak{f}\}$. The fiber over $g K$ can then finally be identified with $\operatorname{Ad}_{g}\left(\mathfrak{f}^{\perp}\right)$.

Summing up: the cotangent bundle to $G / K$ can be identified, given the choice of bilinear form, with pairs $(g K, s)$, with $s \in \operatorname{Ad}_{g}\left(\mathfrak{f}^{\perp}\right)$.

We remark here that in the infinite dimensional situation one must take care about what is meant by the cotangent bundle. If one is not working in the Hilbert space setting then it is usually not the case that a nondegenerate bilinear form gives an identification of $g$ with $\mathfrak{g}^{*}$, but if the form is continuous then it does give a map $\mathfrak{g} \rightarrow \mathfrak{g}^{*}$. In the case that $\mathfrak{g}$ is split into $\mathfrak{f} \oplus \mathfrak{L}$ then $\mathfrak{f}^{\perp}$ gives in general only a subspace of $\mathfrak{Q}^{*}$ and thus the above formulation gives only a subspace of the fiber of the cotangent bundle. We ignore this subtlety in this paper.

3.3. We now apply this to our situation: the holomorphic Grassmannian, which is according to Sect. 2.6 a homogeneous space for the loop group $G l\left(n, H_{\lambda}\right)$ with stabilizer at the origin $G l\left(n, A_{X}^{\text {hol }}\right)$. Since elements of these groups may be considered as holomorphic maps into $G l(n, \mathbb{C})$, we may differentiate pointwise to get the Lie algebras $\mathfrak{g}=g l\left(n, H_{\lambda}\right)$ and $\mathfrak{f}=g l\left(n, A_{X}^{\mathrm{hol}}\right)$. We will identify a complementary subspace for $g l\left(n, A_{X}^{\text {hol }}\right)$ in the Lie algebra $g l\left(n, H_{\lambda}\right)$ and an invariant bilinear form such that with respect to this form $\left(g l\left(n, A_{X}^{\text {hol }}\right)\right)^{\perp}$ is easily identified. To this end we first study a bilinear form on the space $H_{\lambda}$ of germs of holomorphic functions in a deleted neighborhood of $p$.

First recall some basic facts about complex valued harmonic functions of one complex variable. Recall that a smooth function $h$ is harmonic if $\partial \bar{\partial} h=0$. If $h$ is harmonic on a disc, $D$, then $h$ may be written $h=h_{+}+h_{-}$, where $h_{+}$is holomorphic and $h_{-}$is antiholomorphic on $D$. This fact easily follows by noting that $\partial h$ is a closed holomorphic one form. On a punctured disc, $\dot{D}$, it is similarly follows that $h$ may be written in the form $h=h_{+}+h_{-}($on $\dot{D})$ if and only if $\oint_{\partial D} \partial h=0$ (otherwise there is a $\log z \bar{z}$ term). 
We will be interested in global harmonic functions on $X-p$, the set of which we denote by $\operatorname{Har}(X-p)$. A basic fact we need is that given any positive integer $k$, one can construct an harmonic function $h_{k}$ on $X-p$ such that $\left(h_{k}\right)-\lambda^{k}$ is regular at $p$. Furthermore $h_{k}$ is unique up to a constant. The uniqueness follows easily from the fact that the only global harmonic functions on $X$ are constants. Existence comes from looking at the long exact sequence of

$$
0 \rightarrow \operatorname{Har} \rightarrow \operatorname{Har}(n p) \rightarrow \mathcal{O}(n p) / \mathcal{O} \rightarrow 0,
$$

where Har denotes the sheaf of harmonic functions which are regular at $p, \operatorname{Har}(n p)$ is the sheaf of harmonic functions whose behaviour at $p$ is at worst $\lambda^{n}$, and $\mathcal{O}(n p) / \mathcal{O}$ is the skyscraper sheaf of principal parts of meromorphic funtions with poles at $p$ of order at most $n$. In the long exact sequence one must see that $H^{1}(\operatorname{Har}) \simeq H^{1}(\operatorname{Har}(n p)) \simeq \mathbb{C}$ in order to see that $0 \rightarrow H^{0}(\operatorname{Har}) \rightarrow H^{0}(\operatorname{Har}(n p))$ $\rightarrow H^{0}(\mathcal{O}(n p) / \mathcal{O}) \rightarrow 0$ is exact. However $H^{1}$ of these sheaves is easily computed from the short exact sequence

$$
0 \rightarrow \mathcal{O}(n p) \rightarrow \operatorname{Har}(n p) \stackrel{\bar{\partial}}{\longrightarrow} \bar{K} \rightarrow 0
$$

where $\bar{K}$ denotes the sheaf of anti-holomorphic one forms.

Now assume that the genus of $X$ is $g$ and let $\left\{a_{1}, a_{2}, \ldots, a_{g}\right\}$ be the gap-values of $p \in X$, see [GH, p. 273]. For each $a_{i}$ let $h_{a_{i}}$ be the harmonic function on $X-p$ with $h_{a_{i}}-\lambda^{a_{i}+1}$ regular at $p$. Considering the $h_{a_{i}}$ as harmonic functions on $\dot{D}_{\varepsilon}$, set $d\left(h_{\alpha_{i}}\right)_{+}=\tilde{h}_{i} d \lambda$ and let

$$
V=\bigoplus_{1}^{g} \mathbb{C}(\tilde{h})_{+}
$$

\section{Proposition 3.3.1.}

$$
H_{\lambda}=A_{X}^{\text {hol }} \oplus \mathbb{C}\left\{\lambda^{-1}\right\} \lambda^{-1} \oplus V .
$$

Proof. The gaps $a_{i}$ are precisely those integers for which there is no holomorphic function on $X-p$ with pole of order $a_{i}$ at $p$, thus the three subspaces are clearly pairwise disjoint. To see that they span $H_{\lambda}$ define a sheaf $\mathscr{F}:=\mathcal{O}_{X}^{\text {hol }}(X-p)$ $\oplus \mathcal{O}_{X}^{\text {hol }}\left(D_{\varepsilon}\right)$ on the Riemann surface $X$ (with the complex topology). The stalk of $\mathscr{F}$ at $q$ is isomorphic to $\mathcal{O}_{q}^{\text {hol }}$ iff $q \notin \dot{D}_{\varepsilon}$ and to $\mathcal{O}_{q}^{\text {hol }} \oplus \mathcal{O}_{q}^{\text {hol }}$ if $q \in \dot{D}_{\varepsilon}$. Hence we have an exact sequence of sheaves:

$$
0 \rightarrow \mathcal{O}_{X}^{\text {hol }} \rightarrow \mathscr{F} \rightarrow \mathcal{O}_{X}^{\text {hol }}\left(\dot{D}_{\varepsilon}\right) \rightarrow 0
$$

Here on the level of the stalks the injection of $\mathcal{O}_{X}^{\text {hol }}$ is given by $f \mapsto\left(\left.f\right|_{X-p},\left.f\right|_{D_{\varepsilon}}\right)$ and the surjection is given by $(f, g) \mapsto 1 / 2(f-g)$. In cohomology we get:

$$
0 \rightarrow H^{0}\left(X, \mathcal{O}_{X}^{\text {hol }}\right) \rightarrow H^{0}(X, \mathscr{F}) \rightarrow H^{0}\left(X, \mathcal{O}_{X}^{\text {hol }}\left(\dot{D}_{\varepsilon}\right)\right) \rightarrow H^{1}\left(X, \mathcal{O}_{X}^{\text {hol }}\right) \rightarrow 0,
$$

since all the higher cohomology groups vanish $\left(X-p\right.$ and $D_{\varepsilon}$ are Stein). This gives, after taking the limit $\varepsilon \rightarrow 0$ :

$$
0 \rightarrow \mathbb{C} \rightarrow A_{X}^{\text {hol }} \oplus \mathbb{C} \oplus \mathbb{C}\left\{\lambda^{-1}\right\} \lambda^{-1} \rightarrow H_{\lambda} \rightarrow H^{1}\left(X, \mathcal{O}_{X}^{\text {hol }}\right) \rightarrow 0,
$$

where the injection of constants is given by $c \mapsto(c, c, 0)$. Now $H^{1}\left(X, \mathcal{O}_{X}^{\text {hol }}\right) \simeq$ $H^{1}\left(X, \mathcal{O}_{X}\right)$ is $g$ dimensional and one easily checks that $V$ maps injectively to $H^{1}\left(X, \mathcal{O}_{X}\right)$. 
There is another description of the gap-values, namely $a_{i}$ is a gap-value if and only if there is a global holomorphic one-form on $X$ which vanishes at $p$ to precisely the order $a_{i}-1$. For each $i$ let $\omega_{i}$ be a global holomorphic one form with leading term $\lambda^{1-a_{2}} d\left(\lambda^{-1}\right)=-\lambda^{-a_{i}-1} d \lambda$. Locally, on a disc around $p, \omega_{i}=g_{i} d \lambda$ where $g_{i}(\lambda)=-\lambda^{-a_{i}-1}+$ lower order. Let

$$
U=\bigoplus_{1}^{g} \mathbb{C} g_{i}
$$

Note that although the $g_{i}$ 's are not uniquely determined, the space $U$ is independent of the choices, furthermore, if we let $\Omega^{1,0}(X)$ denote the holomorphic one-forms on $X, U$ is isomorphic to $\Omega^{1,0}(X)$.

We now define a bilinear form on $H_{\lambda}$ by

$$
\langle f, g\rangle:=\operatorname{Res}_{p}(f g d \lambda)=\oint f g d \lambda, f, g \in H_{\lambda} .
$$

Since $f$ and $g$ are just germs of holomorphic functions on a deleted neighborhood of $p$ the integral must be interpreted as occurring on the boundary of a small disc $D_{\varepsilon}$ on which representative functions for $f$ and $g$ are defined.

Proposition 3.3.2. With respect to the pairing (3.3.6) we have

$$
\left(A_{X}^{\mathrm{hol}}\right)^{\perp}=A_{X}^{\mathrm{hol}} \oplus V \oplus U
$$

Proof. To see that $A_{X}^{\text {hol }} \oplus V \oplus U \subset\left(A_{X}^{\text {hol }}\right)^{\perp}$ first take $f \in A_{X}^{\text {hol }}$ and $g \in A_{X}^{\text {hol }} \oplus U$. Then $f g d \lambda$ extends to an holomorphic one-form on $X-p$ so it must have zero residue. If instead we take $g \in V$ then $g=h_{+}$for some harmonic function on $X-p$ and $g d \lambda=\partial\left(h_{+}\right)=\partial h$. So by Stokes' theorem $\oint f g d \lambda=\oint f \partial h=\int_{X-D_{\varepsilon}} d(f \partial h)=0$, since $d f=0$ and $d \partial h=\bar{\partial} \partial h=0$.

Conversely, it is a result of A.H. Read [R] (see also [Ro]) that if $\omega$ is a continuous section over $\partial D_{\varepsilon}$ of the holomorphic cotangent bundle, and $\oint_{\partial D_{\varepsilon}} f \omega=0$ for all functions $f$ which are holomorphic on $X-\overline{D_{\varepsilon}}$ and continuous up to the boundary, then $\omega$ extends holomorphically to $X-D_{\varepsilon}$. Using the fact that an holomorphic function on $\overline{X-D_{\varepsilon}}$ can be approximated uniformly by an holomorphic function on $X-p$ (cf. Simha [Si]), and shrinking the neighborhood slightly, it follows that if $\omega$ is an holomorphic one-form on a punctured disc about $p$ and $\oint_{\partial D_{f}} f \omega=0$ for all $f \in \mathcal{O}(X-p)$ then $\omega$ extends to an holomorphic one-form on $X-p$. Hence, from the assumption that $g \in\left(A_{X}^{\text {hol }}\right)^{\perp}$ we conclude that $g d \lambda$ extends to a global holomorphic one-form $\omega$ on $X-p$ satisfying $\oint_{\partial D_{\varepsilon}} \omega=0$. Now note that $\partial: \operatorname{Har}(X-p) \rightarrow \Omega^{1,0}(X-p) / \Omega^{1,0}(X)$ is onto, and that for $h \in \operatorname{Har}(X-p)$, $\partial h=d h_{+}$, so we can identify $\left(A_{X}^{\text {hol }}\right)^{\perp}$ with the holomorphic parts of harmonic functions on $X-p$, i.e., $g \in A_{X}^{\text {hol }} \oplus V \oplus U$.

Note that the direct sums in (3.3.7) is the direct sum of vector spaces. We now extend this result to $\operatorname{Mat}_{n}\left(H_{\lambda}\right)$, the vector space of $n \times n$ matrices with coefficients in $H_{\lambda}$, by defining the bilinear form

$$
\langle A(\lambda), B(\lambda)\rangle:=\oint \operatorname{tr}(A(\lambda) B(\lambda) d \lambda), \quad A(\lambda), B(\lambda) \in \operatorname{Mat}_{n}\left(H_{\lambda}\right) .
$$

It follows immediately from the above proposition that

$$
\operatorname{Mat}_{n}\left(A_{X}^{\mathrm{hol}}\right)^{\perp}=\operatorname{Mat}_{n}\left(A_{X}^{\mathrm{hol}} \oplus V \oplus U\right),
$$


and thus we may identify the fiber of the cotangent bundle of the holomorphic Grassmannian at the point $W^{\text {hol }}=g H_{X}^{n, \text { hol }}$ as the space

$$
T_{W}^{*} G r_{X}^{n}=\operatorname{Ad}_{g}\left(\operatorname{Mat}_{n}\left(A_{X}^{\text {hol }} \oplus V \oplus U\right) .\right.
$$

Let us define

$$
S_{W}^{\mathrm{hol}}=\operatorname{Ad}_{g}\left(g l\left(n, A_{X}^{\mathrm{hol}}\right)\right)
$$

Since, clearly,

$$
S_{W}^{\text {hol }}=\left\{\left.s \in g l\left(n, H_{\lambda}\right)\right|_{s} W^{\text {hol }} \subset W^{\text {hol }}\right\},
$$

we refer to $S_{W}^{\text {hol }}$ as the holomorphic stabilizer algebra of $W^{\text {hol }}$ in $g l\left(n, H_{\lambda}\right)$. Since

$$
T_{W}^{*} G r_{X}^{n}=S_{W}^{\text {hol }} \oplus \operatorname{Ad}_{g}\left(\operatorname{Mat}_{n}(V \oplus U)\right)
$$

we see that the fiber of the cotangent bundle of the Grassmannian at $W$ contains the stabilizer algebra as codimension $2 n^{2} g$ subspace $S_{W}^{\text {hol }}$, where $g$ is the genus of $X$. The $A_{X}^{\text {hol }}$-module $S_{W}^{\text {hol }}$ is just $\operatorname{End}_{A_{X}^{\text {hol }}}\left(W^{\text {hol }}\right)$. Similarly we can introduce $S_{W}^{\mathrm{fin}}=\left\{s \in g l\left(n, L_{\lambda}\right) \mid s W \subset W\right\}$ so that $S_{W}^{\mathrm{fin}}=\operatorname{End}_{A_{X}}(W)$. The relation between the two endomorphism rings is given by:

\section{Lemma 3.3.3.}

$$
S_{W}^{\text {hol }} \simeq A_{X}^{\text {hol }} \otimes S_{W}^{\text {fin }}
$$

Proof. We have an homomorphism

$$
\Phi: A_{X}^{\text {hol }} \otimes \operatorname{End}_{A_{X}}(W) \rightarrow \operatorname{End}_{A_{X}^{\text {hol }}}\left(A_{X}^{\text {hol }} \otimes W\right),
$$

so that the tensor product $b \otimes f$, with $b \in A_{X}^{\text {hol }}, f \in \operatorname{End}_{A_{X}}(W)$, gets mapped to the endomorphism of $W^{\text {hol }}$ that sends $\beta \otimes w$ to $b \beta \otimes f(w)$, where $\beta \in A_{X}^{\text {hol }}, w \in W$. This homomorphism is easily checked to be injective. Note that, as $W^{\text {hol }}$ is free, $\operatorname{End}_{A_{X}^{\text {hol }}}\left(W^{\text {hol }}\right)=g l\left(n, A_{X}^{\text {hol }}\right)$, and that, if $W$ were free, we would have $\operatorname{End}_{A_{X}}(W) \simeq g l\left(n, A_{X}\right)$. Since $g l\left(n, A_{X}^{\text {hol }}\right)=A_{X}^{\text {hol }} \otimes g l\left(n, A_{X}\right)$ in this case $\Phi$ is an isomorphism. For the general case write the projective $A_{X}$-module $W$ as a direct summand, $F=W \oplus W^{\prime}$, of a free module $F$ and put $F^{\text {hol }}=A_{X}^{\text {hol }} \otimes F$. Then any element $s$ of $\operatorname{End}_{A_{X}^{\text {hol }}}\left(W^{\text {hol }}\right)$ can be extended to an endomorphism of $F^{\text {hol }}$ (say by the zero map on the complement of $W^{\text {hol }}$ ) and hence it can be written as $s=\sum f_{i}\left(1 \otimes \tilde{s}_{i}\right)$ with $f_{i} \in A_{X}^{\text {hol }}$ and $\tilde{s}_{i} \in \operatorname{End}(F)$. Let $\pi_{W}: F \rightarrow W$ be the projection on the first summand in the decomposition $F=W \oplus W^{\prime}$ and define $s_{i}=\pi_{W} \circ \tilde{s}_{i} \circ \pi_{W}$; this is an element of $\operatorname{End}(W)$. Then, since tensoring with a fixed module is a functor, $1 \otimes s_{i}=\left(1 \otimes \pi_{W}\right) \circ\left(1 \otimes \tilde{s}_{i}\right) \circ\left(1 \otimes \pi_{W}\right)$. But $1 \otimes \pi_{W}=\pi_{W_{\text {hol }}}$, so $1 \otimes s_{i}$ $=\pi_{W_{\text {hol }}} \circ\left(1 \otimes \tilde{s}_{i}\right) \circ \pi_{W^{\text {hol }}}$ and $\sum f_{i}\left(1 \otimes s_{i}\right)=\pi_{W} \circ \sum f_{i}\left(1 \otimes \tilde{s}_{i}\right) \circ \pi_{W}=\pi_{W} \circ S^{\circ} \pi_{W}$. So we get for $s$, thought of as an endomorphism of $W^{\text {hol }}$, the expression $s=\sum f_{i}\left(1 \otimes s_{i}\right)$ and $\Phi$ is surjective.

In case $W$ is free there is a simple expression for the finite order stabilizer algebra. Recall from 2.6 that $W$ is free iff $W^{\text {hol }}=g H_{X}^{n \text {, hol }}$ for $g$ finite order. Then we have:

Lemma 3.3.4. If $W=g H_{X}^{n \text {, hol }}$ with $g$ a finite order element of $G l\left(n, H_{\lambda}\right)$ then

$$
S_{W}^{\mathrm{fin}}=\operatorname{Ad}_{g}\left(g l\left(n, A_{X}\right)\right) \text {. }
$$

Proof. The holomorphic stabilizer algebra of $H_{X}^{n \text {, hol }}$ is of course $g l\left(n, A_{X}^{\text {hol }}\right)$ and the finite order part is $g l\left(n, A_{X}\right)$. Let $s$ be an element of $S_{W}^{\text {fin }}$, then 
$s W^{\text {hol }}=s g H_{X}^{n \text {, hol }} \subset W^{\text {hol }}=g H_{X}^{n \text {, hol }}$, so $\operatorname{Ad}_{g^{-1}}(s)$ is an element of the finite order stabilizer of $H_{X}^{n, \text { hol }}$, i.e., of $g l\left(n, A_{X}\right)$, as $g$ is assumed to be of finite order. Hence $S_{W}^{\mathrm{fin}} \subset \operatorname{Ad}_{g}\left(g l\left(n, A_{X}\right)\right)$. The reverse inclusion is immediate.

Note that in case $X=\mathbf{P}^{1}$ the situation becomes much simpler: we can identify $S_{W}^{\text {fin }}$ with the fiber at $W$ of the cotangent bundle of the Grassmannian.

We will study stabilizer algebras in more detail in Sect. 6.

\section{Heisenberg Algebras}

4.1. Let $g l\left(r, H_{\lambda}\right)$ be the "real analytic loop algebra on an infinitesimal circle around $p$." Consider the subalgebra $\mathscr{H}^{r}=H_{\lambda}\left[P_{r}\right]$ of $g l\left(r, H_{\lambda}\right)$ consisting of polynomials over $H_{\lambda}$ in the generator $P_{r}$, the $r \times r$ matrix

$$
P_{r}=\left(\begin{array}{cccccc}
0 & 1 & 0 & \ldots & 0 & 0 \\
0 & 0 & 1 & \ldots & 0 & 0 \\
\vdots & \vdots & \vdots & \ddots & \vdots & \vdots \\
0 & 0 & 0 & \ldots & 1 & 0 \\
0 & 0 & 0 & \ldots & 0 & 1 \\
\lambda & 0 & 0 & \ldots & 0 & 0
\end{array}\right)=\lambda E_{r-10}+\sum_{i=0}^{r-2} E_{i i+1}
$$

Note that, since $P_{r}^{r}=\lambda I_{r}$, the general element of $\mathscr{H}^{r}$ may be written in the two distinct forms:

$$
\sum_{i=0}^{r-1} h_{i}(\lambda) P_{r}^{i}, \quad h_{i}(\lambda) \in H_{\lambda},
$$

or,

$$
\sum_{i=-\infty}^{\infty} t_{i} P_{r}^{i}, \quad t_{i} \in \mathbb{C}
$$

$\mathscr{H}^{r}$ is an abelian subalgebra of $g l\left(r, H_{\lambda}\right)$ which extends to the principal Heisenberg subalgebra of the Kac-Moody central extension of $g l\left(r, H_{\lambda}\right)$. In general we will refer to such Abelian subalgebras of $g l\left(r, H_{\lambda}\right)$ also as Heisenberg subalgebras.

Let $n$ be a positive integer and fix a partition $\underline{n}=\left(n_{1} \geqq n_{2} \geqq \ldots n_{k}>0\right)$ of $n$ into $k$ parts. (So we have $n=\sum_{i=1}^{k} n_{i}$.) The Heisenberg subalgebra $\mathscr{H}^{n}$ of type $\underline{n}$ in $\operatorname{gl}\left(r, H_{\lambda}\right)$ is

$$
\mathscr{H}^{\underline{n}}=\bigoplus_{i=1}^{k} \mathscr{H}^{n_{i}} \subset g l\left(r, H_{\lambda}\right),
$$

where each principal Heisenberg subalgebra $\mathscr{H}^{n_{i}}$ is embedded in $g l\left(r, H_{\lambda}\right)$ as a diagonal block, i.e.:

$$
\mathscr{H}^{n}=\left\{\left(\begin{array}{cccc}
H^{n_{1}} & 0 & \ldots & 0 \\
0 & H^{n_{2}} & \ldots & 0 \\
\vdots & \vdots & \ddots & 0 \\
0 & \ldots & \ldots & H^{n_{k}}
\end{array}\right) \mid H^{n_{i} \in \mathscr{H}^{n_{t}}}\right\} .
$$


When the partition $n$ is understood we shall denote by $\mathscr{P}_{a}, 1 \leqq a \leqq k$, the element of $\mathscr{H}^{\underline{n}}$ with $P_{n_{a}}$ in the $a^{\text {th }}$ block and zeroes elsewhere. Thus, the general element of $\mathscr{H}^{n}$ may be written in either of the following forms:

$$
\sum_{i=0}^{r-1} \sum_{a=1}^{k} h_{i}^{a}(\lambda) \mathscr{P}_{a}^{i}, \quad h_{i}^{a}(\lambda) \in H_{\lambda}
$$

or,

$$
\sum_{i=-\infty}^{\infty} \sum_{a=1}^{k} t_{i}^{a} \mathscr{P}_{a}^{i}, \quad t_{i}^{a} \in \mathbb{C} .
$$

The algebras $\mathscr{H}^{n}$ exhaust, up to isomorphism, all maximum Abelian subalgebras of $g l\left(n, H_{\lambda}\right)$ that are stable under the Cartan involution $\omega_{0}: g l(n, \mathbb{C}) \rightarrow g l(n, \mathbb{C})$ given by $X \rightarrow-X^{*}$ (see for instance $[\mathrm{tKr}]$ ).

4.2. We can identify the principal Heisenberg algebra $\mathscr{H}^{r}$ with $H_{z}$ by the $\mathbb{C}$ algebra isomorphism $\mathscr{H}^{r} \rightarrow H_{z}$ given by

$$
\sum_{i=0}^{r-1} h_{i}(\lambda) P_{r}^{i} \mapsto \sum_{i=0}^{r-1} h_{i}\left(z^{r}\right) z^{i} .
$$

This shows that $\mathscr{H}^{r}$ is an integral domain.

The space $H_{\lambda}^{r}$ is an $\mathscr{H}^{r}$-module and the space $H_{z}$ is a $H_{z}$-module. Using the above $\mathbb{C}$-algebra isomorphism there is a module isomorphism $H_{\lambda}^{r} \rightarrow H_{z}$ given by

$$
\sum_{i=0}^{r-1} \alpha_{i}(\lambda) e_{i} \mapsto \sum_{i=0}^{r-1} \alpha_{i}\left(z^{r}\right) z^{i}
$$

Similarly, given a fixed partition $\underline{n}=\left(n_{1} \geqq n_{2} \geqq \ldots n_{k}>0\right)$, we have a $\mathbb{C}$-algebra isomorphism $\mathscr{H}^{\underline{n}} \rightarrow H_{z}^{k}$ by identifying the diagonal $n_{i} \times n_{i}$ block of $\mathscr{H}^{\underline{n}}$ with the $i^{\text {th }}$ component of $H_{z}^{k}$. The ring structure of $H_{z}^{k}$ is given by componentwise multiplication. This also induces a module isomorphism from $H_{\lambda}^{n}$ to $H_{z}^{k}$. Note that arbitrary Heisenberg algebras are not integral domains: they contain zero-divisors.

4.3. The finite order part of $\mathscr{H}^{n}, L_{\bar{\lambda}}^{n}=\bigoplus L_{\lambda}\left[\mathscr{P}_{a}\right]$ is built up out of blocks $L_{\lambda}\left[\mathscr{P}_{a}\right]$ of principal finite order Heisenberg algebras. There is a natural filtration on $L \frac{n}{\lambda}$ which will be important for us in the later sections.

In general if $R$ is a $\mathbb{C}$-algebra an order function on $R$ is a map $\mathfrak{D}: R \rightarrow \mathbb{Z}$ such that $\mathfrak{o}\left(r_{1} r_{2}\right) \leqq \mathfrak{o}\left(r_{1}\right) \mathfrak{D}\left(r_{2}\right)$ and $\mathfrak{D}(c r)=\mathfrak{o}(r)$ for all $r, r_{1}, r_{2} \in R, c \in \mathbb{C}$. Given an order function we define a filtration . . $R_{i} \subset R_{i+1} \subset \ldots$ of $R$ by declaring $R_{i}$ to be the set of elements $r$ of $R$ that have order $\mathfrak{o}(r)$ less than or equal $i$.

On the ring of Laurent series we define an order by $\mathfrak{p}(f(\lambda))=k$ if $f(\lambda)=\sum_{-\infty}^{k} f_{i} \lambda^{i}, f_{k} \neq 0$. Define then on the finite order principal Heisenberg algebra $L_{\lambda}\left[P_{n}\right]$ an order $\mathrm{o}_{n}$ by giving the generator $P_{n}$ order 1 and $\lambda$ order $n$. In other words if $s \in L_{\lambda}\left[P_{n}\right]$ has an expansion $s=\sum_{i=0}^{n-1} f_{i}(\lambda) P_{n}^{i}$ then $\mathfrak{o}_{n}(s)=$ $\max _{i}\left(n \cdot \mathfrak{o}\left(f_{i}(\lambda)\right)+i\right)$. Note that this filtration corresponds to the natural filtration on $L_{z} \subset H_{z}$ under the isomorphism $\mathscr{H}^{n} \rightarrow H_{z}$ of Eq. (4.2.1). define

Finally for $s$ an element of an arbitrary finite order Heisenberg algebra $L_{\bar{\lambda}}^{n}$ we

$$
\mathfrak{b}_{\underline{n}}=\max _{i=1}^{k} \mathfrak{b}_{n_{i}}\left[\pi_{i}(s)\right],
$$

where

$$
\pi_{i}: \mathscr{H}^{\underline{n}} \rightarrow \mathscr{H}^{n_{i}}
$$


is the projection of the $i^{\text {th }}$ block of size $n_{i} \times n_{i}$ in the Heisenberg algebra. Let us denote by $L_{s}$ the elements of order $s$ in $L_{\bar{\lambda}}^{\underline{n}}$. Then we have $L_{s} \subset L_{s+1}$ and $L_{s+1} / L_{s} \simeq \mathbb{C}^{k}$, where $k$ is the number of parts of $\underline{n}$.

\section{Covering Maps and Direct Images}

5.1. Our Grassmannians $G r_{X}^{n}$ are constructed from rank $n$ bundles $\mathscr{E}$ on the curve $X$. Now it is known that "generically" such bundles are the direct image of a line bundle under an $n$-sheeted covering map $f: Y \rightarrow X$, where $Y$ is a smooth curve; for a precise formulation see [BNR]. We will consider more generally curves $Y$ that may be singular, reducible or even decomposable. This makes it then natural to consider also torsion free coherent sheaves, not only bundles. For the reader's convenience we will include some of the standard defintions and results; for more details see e.g., [Ha].

5.2. Let $Y$ be an algebraic projective curve and $\mathcal{O}_{Y}$ its algebraic structure sheaf. An algebraic coherent sheaf $\mathscr{F}$ is a sheaf of $\mathcal{O}_{Y}$-modules that is locally the cokernel of a morphism of free finite modules: we have for every $q \in Y$ a subset $U$ containing $q$ and an exact sequence, for some nonnegative integers $\alpha, \beta$ :

$$
\mathcal{O}_{Y}(U)^{\alpha} \rightarrow \mathcal{O}_{Y}(U)^{\beta} \rightarrow \mathscr{F}(U) \rightarrow 0 .
$$

A coherent sheaf $\mathscr{F}$ is called torsion-free if $s \cdot f=0, s \in \mathcal{O}_{Y, q}, f \neq 0 \in \mathscr{F}_{q}$, with $s$ not a zero divisor, implies $s=0$. We will always assume sheaves to be coherent and torsion-free in the sequel.

The rank of $\mathscr{F}$ at $q$ is the maximal number of linearly independent elements (over $\mathcal{O}_{Y, q}$ ) in $\mathscr{F}_{q}$. The rank of a coherent sheaf is constant inside every irreducible component of $Y$. Another local invariant of $\mathscr{F}$ is the fiber dimension $\mu_{q}(\mathscr{F})$ at a closed point $q$ defined as follows: let $\mathrm{m}_{q} \subset \mathcal{O}_{\mathrm{Y}, q}$ be the maximal ideal of $q$ and $K_{q}=\mathcal{O}_{Y, q} / \mathfrak{m}_{q} \simeq \mathbb{C}$ the local residue field. Then we put $\mu_{q}(\mathscr{F})=\operatorname{dim}_{K_{q}}\left(\mathscr{F}_{q} / \mathfrak{m}_{q} \mathscr{F}_{q}\right)$. By Nakayama's lemma this is also the minimal number of generators of $\mathscr{F}_{q}$; in other words the fiber dimension is the minimal $\beta$ that can occur in the sequence (5.2.1). The rank of $\mathscr{F}$ and the fiber dimension of $\mathscr{F}$ at $q$ will be equal if and only if $\mathscr{F}_{q}$ is free. If $q$ is a nonsingular point the stalk $\mathscr{O}_{Y, q}$ is a Dedekind domain. Since a finitely generated torsion-free module over a Dedekind domain is locally free (see [Bo, VII, Sect. 5.10]) in the case of a smooth point the stalk of $\mathscr{F}$ is free. In particular if $Y$ is a nonsingular irreducible curve every torsion free coherent sheaf is locally free, i.e., the sheaf of sections of an algebraic vector bundle.

5.3. Continue with $X$ being an irreducible, reduced, nonsingular, projective curve and $Y$ an arbitrary reduced projective curve. An l-fold branched cover of $X$ by $Y$ is a morphism $f: Y \rightarrow X$ such that for every $x \in X$ the inverse image $f^{-1}(x)$ consists of a finite number of points $\left\{y_{1}, y_{2}, \ldots, y_{k}\right\}$, with $k=\ell$ for all but a finite number of $x$, and such that the rank of $\mathcal{O}_{Y, y_{i}}\left(\operatorname{over} \mathcal{O}_{X, x}\right)$ is $\ell_{i}$, with $\sum_{1}^{k} \ell_{i}=\ell$. Here we use the local homomorphism

$$
f_{y_{i}}^{\#}: \mathcal{O}_{X, x} \rightarrow \mathcal{O}_{Y, y_{i}}
$$

to give $\mathcal{O}_{Y, y_{i}}$ the structure of an $\mathcal{O}_{X, x}$-module. We will refer to the rank $\ell_{i}$ as the ramification index of the covering map at $y_{i}$. 
Let now $\mathscr{F}$ be a sheaf (i.e., a coherent torsion-free sheaf) on $Y$, of rank $m_{i}$ at the $i^{\text {th }}$ point lying over $x \in X$. We don't assume these $m_{i}$ to be constant or even non-zero. Then the direct image sheaf $f_{*} \mathscr{F}$ on $X$ is defined by $f_{*}$ $\mathscr{F}(U):=\mathscr{F}\left(f^{-1}(U)\right)$; the structure of $\mathcal{O}_{X}(U)$ module is again provided by using the homomorphism $f^{\#}$. The direct image sheaf is then a coherent torsion free sheaf of rank $n=\sum_{i} l_{i} m_{i}$ (this is independent of the choice of the point $x$ ) on $X$, and since $X$ is non-singular this is in fact a rank $n$ vector bundle.

In this way we obtain from sheaves $\mathscr{F}$ on $Y$ vector bundles $\mathscr{E}=f_{*} \mathscr{F}$ on $X$. Next we investigate how to obtain the other ingredient going into the concoction of an element of $G r_{X}^{n}$ : the local trivialization $t$ of $\mathscr{E}$ at the point $p \in X$.

5.4. If $\left(Y, \mathcal{O}_{Y}\right)$ is an algebraic curve, there exists a unique analytic space $\left(Y, \mathcal{O}_{Y}^{\text {hol }}\right)$ associated to it and for any algebraic coherent sheaf $\mathscr{F}$ we have a unique analytic coherent sheaf $\mathscr{F}^{\text {hol }}=\mathcal{O}_{Y}^{\text {hol }} \otimes \mathscr{F}$. If $f: Y \rightarrow X$ is an algebraic $\ell$-fold branched cover we can extend it to the corresponding analytic spaces and $f_{*} \mathscr{F}^{\text {hol }}$ is for any coherent sheaf $\mathscr{F}$ an analytic coherent sheaf on $\left(X, \mathcal{O}_{X}^{\text {hol }}\right)$. If $X$ is nonsingular this is the sheaf of sections of an holomorphic bundle on $X$.

We will be interested in the situation where the fiber $f^{-1}(p)=\left\{p_{1}, \ldots, p_{k}\right\}$ of the special point $p$ on $X$ contains only nonsingular points. In this case the stalk $\left(f_{*} \mathscr{F}^{\text {hol }}\right)_{p}$ can be described very explicitly. If $\lambda^{-1}$ is a local holomorphic coordinate at $p$ then $f_{p_{i}}^{\#}\left(\lambda^{-1}\right)$ belongs to $m_{p_{i}}^{\ell_{i}}$ but not to $m_{p_{i}}^{\ell_{i}+1}$, where $\ell_{i}$ is the ramification index at $p_{i}$. By choosing a local holomorphic coordinate $z_{i}$ at $p_{i}$ appropriately we may assume that $f_{p_{i}}^{\#}\left(\lambda^{-1}\right)=z_{i}^{\ell_{i}}$. Then a basis for $\mathcal{O}_{Y, p_{i}}$ as a $\mathcal{O}_{X, p}$ module is provided by $\left\{1, z_{i}, z_{i}^{2}, \ldots, z_{i}^{\ell_{2}-1}\right\}$. If $\mathscr{F}$ has rank $m_{i}$ at $p_{i}$ then we can choose a basis $\left\{f_{1}^{(i)}, f_{2}^{(i)}, \ldots, f_{m_{\imath}}^{(i)}\right\}$ for $\mathscr{F}_{p_{\imath}}$ over $\mathcal{O}_{Y, p_{\imath}}$ and doing this at all points of the fiber $f^{-1}(p)$ will give a basis of $\left(f_{*} \mathscr{F}\right)_{p}$ over $\mathcal{O}_{X, p}$, viz.,

$$
\left\{z_{i}^{\beta} f_{\gamma}^{(i)} \mid 1 \leqq \gamma \leqq m_{i}, 0 \leqq \beta \leqq \ell_{i}-1,1 \leqq i \leqq k\right\}
$$

Summarizing, the following

Geometric Data.

(1) An $\ell$-fold branched covering map $f: Y \rightarrow X$, where $Y$ is an arbitrary projective reduced curve and $X$ is a smooth integral projective curve, such that $f^{-1}(p)$ consists of $k$ nonsingular points $p_{i}$ with ramification indices $\ell_{i}$,

(2) Local holomorphic coordinates $z_{i}$ at $p_{i}$ such that $z_{i}^{\ell_{2}}=f_{p_{i}}^{\#}\left(\lambda^{-1}\right)$,

(3) A torsion-free coherent sheaf $\mathscr{F}$ of rank $m_{i}$ at $p_{i}$ with a basis of $\mathscr{F}_{p_{i}}$ at each point $p_{i}$

determine an element $[\mathscr{E}, t]$ of the Grassmannian $G r_{X}^{n}$, where $n=\sum \ell_{i} m_{i}$ : namely, put $\mathscr{E}=f_{*} \mathscr{F}$ and construct the trivialization from the basis (5.4.1).

The equivalent description of $[\mathscr{E}, t] \in G r_{X}^{n}$ in terms of an $A_{X}$ submodule $W$ of $L_{\lambda}^{n}$ is obtained from the geometric data as follows: let $\sigma$ be a section of $\mathscr{F}$ over $Y-f^{-1}(p)$, i.e., $\sigma \in H^{0}\left(Y-f^{-1}(p), \mathscr{F}\right)$. Then $\sigma$ determines a section of the rank $n$ bundle $\mathscr{E}=f_{*} \mathscr{F}$ over $X-p$ and using the basis (5.4.1) and the local coordinate $\lambda^{-1}$ we obtain, an element of $L_{\lambda}^{n}$ as in Sect. (2.3). The space $W \subset L_{\lambda}^{n}$ is the collection of elements of $L_{\lambda}^{n}$ obtained in this way from sections of $H^{0}\left(Y-f^{-1}(p), \mathscr{F}\right)$. Similarly $W^{\text {hol }}$ is obtained by pushing down the sections of $H^{0}\left(Y-f^{-1}(p), \mathscr{F}^{\text {hol }}\right)$ and again using the basis (5.4.1) to obtain an element of $H_{\lambda}^{n}$.

The simplest case of this construction is that where the sheaf $\mathscr{F}$ has rank 1 everywhere on $Y$. Then the covering map $f: Y \rightarrow X$ has degree $n$ and the partition of $n$ that defines the relevant Heisenberg algebra is given by the ramification indices 
at the $p_{i}$. The simplest subcase here is maybe that where there is a single point lying over $p \in X$ and the associated Heisenberg is the principal Heisenberg of size $n$. This is the situation that leads to the $\mathrm{KdV}$ hierarchies, in case $X=\mathbf{P}^{1}$, cf. [SW].

5.5. In this subsection we study the stabilizer algebra of the element $W \in G r_{X}^{n}$ which we obtain from the above geometric data. Recall from Sect. 3.3 that the stabilizer algebra of $W \in G r_{X}^{n}$, is given by $S_{W}^{\text {hol }}=\left\{s \in g l\left(n, H_{\lambda}\right) \mid s W^{\text {hol }} \subset W^{\text {hol }}\right\}$. Now notice that multiplication by an holomorphic funtion $\tilde{s}$ on $Y-f^{-1}(p)$ preserves the space $H^{0}\left(Y-f^{-1}(p), \mathscr{F}^{\text {hol }}\right)$ of holomorphic sections. Thus the pushforward $s=f_{*}(\tilde{s})$ gives an element of $S_{W}^{\text {hol }}$ when expanded in the basis (5.4.1). Indeed we will argue that $s$ belongs to the intersection of this stabilizer algebra and an Heisenberg algebra corresponding to a partition of $n$ to be determined below.

First observe that the action of the element $z_{i}$ on $\mathcal{O}_{Y, p_{i}}$, considered as an $\mathcal{O}_{X, p}$-module with basis $\left\{1, z_{i}, z_{i}^{2}, \ldots, z_{i}^{l_{i}-1}\right\}$, is given with respect to this basis by multiplication by the matrix:

$$
\left(\begin{array}{cccccc}
0 & 0 & \ldots & 0 & 0 & \lambda^{-1} \\
1 & 0 & \ldots & 0 & 0 & 0 \\
0 & 1 & \ldots & 0 & 0 & 0 \\
\vdots & \vdots & \ddots & \vdots & \vdots & \vdots \\
0 & 0 & \ldots & 1 & 0 & 0 \\
0 & 0 & \ldots & 0 & 1 & 0
\end{array}\right)
$$

i.e., $z_{i}$ is represented by the inverse of the generator of the principal Heisenberg algebra of size $\ell_{i}$, see Sect. 4.4. The basis $\left\{1, z_{i}, z_{i}^{2}, \ldots, z_{i}^{l_{i}-1}\right\}$ of $\mathcal{O}_{Y, p_{i}}$ as $\mathcal{O}_{X, p}$ module, along with a basis $\left\{f_{0}^{i}, f_{1}^{i}, \ldots, f_{m_{i}-1}^{i}\right\}$ of $\mathscr{F}_{p_{i}}$ as $\mathcal{O}_{Y, p_{l}}$-module, induces a basis of $\mathscr{F}_{p_{i}}$ as $\mathcal{O}_{X, p}$ module, namely;

$$
\left\{f_{0}^{i}, f_{1}^{i}, \ldots, f_{m_{i}-1}^{i}, z_{i} f_{0}^{i}, z_{i} f_{1}^{i}, \ldots, z_{i} f_{m_{i}-1}^{i}, \ldots, z_{i}^{l_{i}-1} f_{0}^{i}, z_{i}^{l_{i}-1} f_{1}^{i}, \ldots, z_{i}^{l_{i}-1} f_{m_{2}-1}^{i}\right\} .
$$

With respect to this basis the action of $z_{i}^{-1}$ on $\mathscr{F}_{p_{i}}$ is represented by the element $P_{l_{i} m_{i}}^{m_{i}}$, the $m_{i}^{\text {th }}$ power of the generator of the principal Heisenberg algebra of size $l_{i} m_{i}$.

Next observe that any holomorphic function $\tilde{s}$ on $Y-f^{-1}(p)$ may be expanded in terms of the local coordinates at the points $p_{i}$ over $p$ to give a $k$-tuple $\left\{s_{1}\left(z_{1}\right), s_{2}\left(z_{2}\right), \ldots, s_{k}\left(z_{k}\right)\right\}$, with $s_{i}\left(z_{i}\right) \in H_{z_{i}^{-1}}$. Hence in the pushforward, $s=f_{*}(\tilde{s})$, each component $s_{i}\left(z_{i}\right)$ is represented as an expansion in the element $P_{m_{l} l_{i}}^{m_{i}}$ of the principal Heisenberg algebra $\mathscr{H}^{m_{2} l_{2}}$. Putting this all together we get a representation for $\tilde{s}$ as an element of $\mathscr{H}^{\underline{n}}$, where $\underline{n}$ is the partition of $n=m_{1} l_{1}+m_{2} l_{2}+\cdots$ $+m_{k} l_{k}$ into the $k$ parts $m_{i} l_{i}$. So we find, as promised, that the pushforward of a holomorphic function on $Y-f^{-1}(p)$ belongs to $S_{W}^{\text {hol }} \cap \mathscr{H}^{\stackrel{n}{n}}$. In fact:

Lemma 5.5.1. Let $W$ be a point of the Grassmannian given by the geometric data in Sect. 5.4. Then

$$
f_{*}\left(H^{0}\left(Y-f^{-1}(p), \mathcal{O}_{Y}^{\text {hol }}\right)\right)=S_{W}^{\text {hol }} \cap \mathscr{H}^{\underline{n}} .
$$

Proof. It remains to be shown that $\left(S_{W}^{\text {hol }} \cap \mathscr{H}^{n}\right) \subset f_{*}\left(H^{0}\left(Y-f^{-1}(p), \mathcal{O}_{Y}^{\text {hol }}\right)\right)$. Let $s \in S_{W}^{\text {hol }} \cap \mathscr{H}^{\underline{n}}$. Using the isomorphism $\mathscr{H}^{\underline{n}} \rightarrow H_{z}^{k}$ induced by the partition $\underline{n}$ into 
$k$ parts (see Sect. 4.2) and identifying $H_{z^{-1}}^{k}$ with $\bigoplus_{i=1}^{k} H_{z_{i}^{-1}}$ we see that $s$ can be thought of as an holomorphic function $\tilde{s}$ in a deleted neighborhood of $f^{-1}(p)$. Since $s \in S_{W}^{\text {hol }}$ the function $\tilde{s}$ stabilizes $H^{0}\left(Y-f^{-1}(p), \mathscr{F}\right)$, and therefore $\tilde{s}$ extends to an holomorphic function on all of $Y-f^{-1}(p)$. (We use here the fact that $H^{0}\left(Y-f^{-1}(p), \mathscr{F}\right)$ is free. $)$

It follows then that we also have:

$$
f_{*}\left(H^{0}\left(Y-f^{-1}(p), \mathcal{O}_{Y}\right)\right)=S_{W}^{\mathrm{fin}} \cap \mathscr{H}^{\underline{n}} .
$$

Note that we have made here a choice of ordering of the basis (5.4.1) to represent holomorphic functions by explicit matrices. The choice we made seems the most natural; it has the fortunate effect that for a covering map $f: Y \rightarrow X$ with $k$ points over $p \in X$ the holomorphic functions on $Y-f^{-1}(p)$ corresponds to a subalgebra of an Heisenberg algebra determined by a partition with also $k$ parts. However other choices are possible and this will give the holomorphic functions as subalgebras of more complicated Heisenbergs. For instance we might take as basis for $\mathscr{F}_{p_{i}}$ :

$$
\left\{f_{0}^{i}, z_{i} f_{0}^{i}, \ldots, z_{i}^{l_{i}-1} f_{0}^{i}, f_{1}^{i}, z_{i} f_{1}^{i}, \ldots, z_{i}^{l_{i}-1} f_{1}^{i}, \ldots, f_{m_{2}-1}^{i}, z_{i} f_{m_{2}-1}^{i}, \ldots, z_{i}^{l_{l}-1} f_{m_{i}-1}^{i}\right\} .
$$

In this case $z_{i}^{-1}$ acts on $\mathscr{F}_{p_{i}}$ as the matrix $\operatorname{diag}\left(P_{l_{i}}, P_{l_{l}}, \ldots, P_{l_{i}}\right)\left(m_{i}\right.$ terms) and a holomorphic section will be represented using this ordering at all points $p_{i}$ by a matrix from the Heisenberg algebra corresponding to the partition $n=l_{1}+\cdots+l_{1}+l_{2}+\cdots+l_{2}+\cdots+l_{k}+\cdots+l_{k}$ (every $l_{i}$ repeated $m_{i}$ times). Also intermediate conventions for the ordering can of course be chosen. In the direct Krichever problem (obtaining a point in the infinite Grassmannian from geometric data) we can choose not to use these artificially complicated Heisenbergs, but in the inverse process of obtaining geometric data from a point in the Grassmannian we can't avoid them. This is the reason that Theorem 7.5.1 is rather complicated.

5.6. This ring $S_{W}^{n}=S_{W}^{\text {fin }} \cap \mathscr{H}^{n}$, where $W$ comes from the geometric data in (5.4) is, as we have seen, isomorphic to the ring $A_{Y}$ of rational functions on $Y$ regular except possibly at points in $f^{-1}(p)$. We have a filtration

$$
A_{Y}=A_{0} \subset A_{1} \subset A_{2} \subset \ldots, A_{s}=H^{0}\left(Y, \mathcal{O}_{Y}(s D)\right),
$$

where $D=p_{1}+p_{2}+\cdots+p_{k}$. So $A_{s}$ consists of the regular functions on $Y-f^{-1}(p)$ that have at most a pole of order $s$ at any of the points in $f^{-1}(p)$. By Riemann-Roch we have for large $s$ :

$$
A_{s+1} / A_{s} \simeq \mathbb{C}^{k}
$$

In Sect. 4.3 we introduced a filtration on the finite order Heisenberg algebra $L \frac{n}{\lambda}=\bigoplus_{i=1}^{k} L_{\lambda}\left[\mathscr{P}_{n_{i}}\right]$. We have to change this slightly to obtain a filtration on $S_{W}^{n} \subset \oplus L_{\lambda}\left[\mathscr{P}_{n_{i}}\right]$ that corresponds to the filtration on $A_{Y}$. We saw that $z_{i}^{-1}$ corresponds to the element $\mathscr{P}_{\ell_{i} m_{2}}^{m_{i}}$. Since $z_{i}^{-1}$ has a single pole at $p_{i}$ we should give $\mathscr{P}_{\ell_{i} m_{i}}^{m_{i}}$ order 1 , not $m_{i}$ as in Sect. 4.3.

In other words if $n_{i}=\ell_{i} m_{i}$ we define on the subalgebra

$$
L_{\lambda}\left[\mathscr{P}_{\ell_{i} m_{i}}^{m_{i}}\right] \subset L_{\lambda}\left[\mathscr{P}_{\ell_{\imath} m_{l}}\right]
$$

a modified order function

$$
\mathfrak{o}_{n_{i}, \ell_{1}}(s)=\mathfrak{v}_{i}(s) / m_{i}, \quad s \in L_{\lambda}\left[\mathscr{P}_{\ell_{1} m_{l}}^{m_{i}}\right] .
$$


Then, on the subalgebra

$$
L_{\bar{\lambda}}^{\underline{n}, \underline{\ell}}=\bigoplus_{i=1}^{k} L_{\lambda}\left[\mathscr{P}_{\ell_{2} m_{i}}^{m_{l}}\right]
$$

define the order

$$
\mathfrak{o}_{\underline{n}, \underline{\ell}}(s)=\max _{1}^{k}\left[\mathfrak{o}_{n_{i}, \ell}\left(\pi_{i}(s)\right)\right], \quad s \in L_{\bar{\lambda}}^{\underline{n}, \underline{\ell}} .
$$

With this new definition of the filtration the correspondence of $A_{Y}$ and $S_{W}^{n}$ is an isomorphism of filtered $\mathbb{C}$-algebras.

\section{Stabilizer Algebras and Their Spectra}

6.1. Let $W$ be an element of $G r_{X}^{n}$ and recall from Sect. 3.3 that the stabilizer algebra of $W^{\text {hol }}$ is

$$
S_{W}^{\text {hol }}=\left\{s \in g l\left(n, H_{\lambda}\right) \mid s W^{\text {hol }} \subset W^{\text {hol }}\right\} .
$$

This is a noncommutative algebra associated to $W$ which can be identified with a large subspace of the fiber of the cotangent bundle of the Grassmannian at $W$. We saw in the previous section that for $W$ coming from a covering map $f: Y \rightarrow X$, and additional data, the commutative subalgebra $S_{W}^{\text {hol }} \cap \mathscr{H}^{n}$ has a natural geometric interpretation: it is essentially the ring of holomorphic functions on the analytic space $Y-f^{-1}(p)$. In the same way the finite order part $S_{W}^{\text {fin }} \cap \mathscr{H}^{n}$ can be identified with the coordinate ring of the affine curve $Y-f^{-1}(p)$.

Wanting to obtain the geometric data from $W$ we define for arbitrary $W$ (not a priori coming from the Geometric Data of Sect. 5.4) and arbitrary partition of $n$ :

$$
S_{W}^{n}=S_{W}^{\mathrm{fin}} \cap \mathscr{H}^{\underline{n}} .
$$

For brevity we refer to this as the stabilizer algebra of type $\underline{n}$. This is an abelian subalgebra of the finite order stabilizer $S_{W}^{\text {fin }}$ which in general is not maximal. We will see that $S_{W}^{n}$ is the affine coordinate ring of a curve, $\operatorname{Spec}\left(S_{W}^{n}\right)$, covering $X$. In Sect. 7 we will construct a compactification for $\operatorname{Spec}\left(S_{W}^{n}\right)$ and we will show that we can in fact reconstruct all the data from $W$ and the choice of Heisenberg algebra $\mathscr{H}^{n}$.

6.2. Example. Although $\mathscr{H}^{n}$ is a maximal Abelian subalgebra of $g l\left(n, H_{\lambda}\right)$, it is in general not true that the stabilizer $S_{\bar{W}}^{n}$ is maximal Abelian in $S_{W}^{\mathrm{fin}}$. For example let $W \in G r_{X}^{2}$ have a basis over $A_{X}$ consisting of $w_{1}=\left(\begin{array}{l}1 \\ b\end{array}\right), w_{2}=\left(\begin{array}{l}0 \\ 1\end{array}\right)$, where $b \notin K_{X}$, for $K_{X}$ the quotient field of $A_{X}$. Consider then the principal stabilizer subalgebra $S_{W}^{2}$, corresponding to the partition of 2 into one part. It consists of polynomials over $L_{\lambda}$ in the matrix $P_{2}=\left(\begin{array}{ll}0 & 1 \\ \lambda & 0\end{array}\right)$ stabilizing $W$, since $W$ is free over $A_{X}$. But for any $f(\lambda) \in L_{\lambda}$ we have $f(\lambda) P_{2} w_{2} \notin W$, since clearly no element of $W$ has a zero as second component. Hence $S_{W}^{2}=A_{X} \cdot 1_{2 \times 2}$. This means that any element of $S_{W}$ commutes with $S_{W}^{2}$, which is therefore not maximal.

We can also use this example to point out that, despite Lemma 3.3.3, it is not in general true that the holomorphic stabilizer of type $n, S_{W}^{n}$, hol , is obtained by tensoring the finite order stabilizer of type $\underline{n}, S_{\bar{W}}^{n}$, with $A_{X}^{\overline{\mathrm{hol}}}$. Indeed, if we take for 
simplicity $X=\mathbf{P}^{1}$ and $b$ above to be of the form $h / g$, where $h$ and $g$ are holomorphic on $X-p$ and have an essential singularity at $p$, then the ring

$$
R=A_{X}^{\text {hol }} \cdot 1_{2 \times 2} \oplus A_{X}^{\text {hol }} \cdot f(\lambda)\left(\begin{array}{ll}
0 & 1 \\
\lambda & 0
\end{array}\right)
$$

certainly belongs to $S_{W}^{2, \text { hol }}$ if $f(\lambda)=g^{2}(\lambda)$. So $S_{W}^{2, \text { hol }}$ is strictly bigger than $A_{X}^{\mathrm{hol}} \otimes S_{W}^{2}=A_{X}^{\mathrm{hol}} \cdot 1_{2 \times 2}$. Note that in this example $\operatorname{Spec}\left(S_{W}^{2}\right)$ is just $X-p$, but that $R$ can be thought of as the ring of analytic functions of the "hyperelliptic analytic curve" $z^{2}=\lambda(g(\lambda))^{4}$. In general, it seems that to study the holomorphic stabilizer algebra $S_{\frac{W}{W}}^{n \text {, hol }}$ one has to introduce infinite genus curves or similar complicated objects [McK].

6.3. Let us list some algebraic properties of the finite order stabilizer algebra $S_{W}^{n}$. It is a reduced ring, i.e., it does not contain any nilpotents, and it is torsion free as $A_{X}$-module. Furthermore, $W$ is finitely generated and projective, so there is a free finite rank $A_{X}$ module $F$ and an other module $W^{\prime}$ with $F=W \oplus W^{\prime}$. Thus $S_{W}^{\text {fin }}(=\operatorname{End}(W))$, and hence $S_{W}^{n}$, is an $A_{X}$-submodule of the endomorphism ring $\operatorname{End}(F)$, which is itself of finite rank and free. Since $A_{X}$ is Noetherian it follows that $S_{W}^{n}$ is finitely generated and Noetherian. Finally, since the ring $A_{X}$ is always contained as a diagonal subring in $S_{W}^{n}$, it follows that $S_{W}^{n}$ is an integral extension of $A_{X}$.

This last fact can be seen very explicitly in the case $W$ is free: let $s \in S_{W}^{n}$, then $s$ is conjugate to an element of $g l\left(n, A_{X}\right)$ (see Lemma 3.3.4). Hence the characteristic polynomial of $s, P_{s}(t)=\operatorname{det}\left(t \mathbf{1}_{n}-s\right)$ belongs to $A_{X}[t]$, and is of the form

$$
t^{n}+a_{1} t^{n-1}+\cdots+a_{n}=0, \quad a_{i} \in A_{X} .
$$

Since $s$ satisfies, by the Cayley-Hamilton theorem, its own characteristic equation we see that $s$ is integral over $A_{X}$.

6.4. Associated to a commutative $\operatorname{ring} A$ is a topological space $\operatorname{Spec}(A)$, the set of prime ideals in $A$, with as basis of open sets $D(f)=\{p \in \operatorname{Spec}(A) \mid f \notin p\}$, where $f \in A$. $X=\operatorname{Spec}(A)$ comes naturally equipped with a sheaf of local rings, the structure sheaf $\mathcal{O}_{X}$. The space of sections of the structure sheaf over the open set $D(f)$ is the localization $A_{f}$ and the stalk at a point $p$ of $X$ is the local ring $\mathcal{O}_{X, p}=A_{p}$. For instance $\operatorname{Spec}\left(A_{X}\right)$ is the affine curve $X-p$ together with its sheaf of regular functions. Here we want to consider the $\operatorname{space} \operatorname{Spec}\left(S_{W}^{n}\right)$.

Since the commutative ring $S_{W}^{n}$ is an integral extension of $A_{X}$, the Krull dimensions of $S_{\bar{W}}^{n}$ and $A_{X}$ are equal and hence also $\operatorname{Spec}\left(S_{\bar{W}}^{n}\right)$ is a curve.

As a topological space $\operatorname{Spec}\left(S_{W}^{n}\right)$ decomposes into a finite number of irreducible components $V\left(\mathfrak{p}_{a}\right)=\left\{p \in \operatorname{Spec}(A) \mid \mathfrak{p}_{a} \subset p\right\}$, where the $\mathfrak{p}_{a}$ are the minimal primes of $S_{W}^{n}, a=1, \ldots, r$. The elements of the minimal primes $\mathfrak{p}_{a}$ are precisely the zero divisors of $S_{W}^{n}$. Since $S_{W}^{n}$ is a subring of an Heisenberg algebra the zero-divisors are easy to identify using the block structure and so we can determine the minimal primes and hence the decomposition of $\operatorname{Spec}\left(S_{W}^{n}\right)$ into irreducible components.

Recall from Sect. 3.2 that a principal Heisenberg algebra (corresponding to a partition of $n$ into one part) is an integral domain. Hence also a stabilizer algebra $S_{W}^{n}$ of principal type is an integral domain and in this case $\langle 0\rangle$ is the unique minimal prime and $\operatorname{Spec}\left(S_{W}^{n}\right)$ is irreducible. (This is the situation one usually finds discussed in the literature on the Krichever method in soliton theory, where one studies the $\mathrm{KdV}$ or KP hierarchies related to the principal Heisenberg algebra, see e.g., $[\mathrm{SeW}]$.) 
To study the general case of a stabilizer algebra $S_{W}^{n}$ contained in a non-principal Heisenberg, we use the projections $\pi_{i}$ introduced in (4.3.2). Let $s \in S_{W}^{n}, \underline{n}$ a partition of $n$ into $k>1$ parts, so $\mathscr{H}^{\underline{n}}$ is not an integral domain in this case, although $S_{W}^{n}$ might be. Then we define the support of $s$ as the subset

$$
S(s)=\left\{i \in I \mid \pi_{i}(s) \neq \emptyset\right\}, I=\{1,2, \ldots, k\} .
$$

We say that $s$ has full support if $S(s)=I$. If $s$ and $t$ are two elements in $S_{\bar{W}}^{n}$ then $S(s t)=S(s) \cap S(t)$ since the principal Heisenberg algebras are integral domains. Thus it follows that if $s$ has full support then it can not be a zero-divisor. In fact from the following lemma we see that $s$ is a zero-divisor if and only if it does not have full support:

Lemma 6.4.1. Let $s \in S_{\bar{W}}^{n}$, Then there exists a $t \in S_{\bar{W}}^{n}$ with $S(t)=I-S(s)$ and hence $s t=0$.

Proof. Consider the projection $\pi_{S(s)}=\bigoplus_{i \in S(s)} \pi_{i}$. Then $\pi_{S(s)}(s)=s$ is a non-zerodivisor in $\pi_{S(s)}\left(S_{W}^{n}\right)$. Now $\pi_{S(s)}\left(S_{W}^{n}\right)$ is an integral extension of $A_{X}$ (since $S_{W}^{n}$ is) and hence we have an equation for $\pi_{S(s)}(s)$ of the form $\left(\pi_{S(s)}(s)\right)^{n}+\sum_{i} a_{i}\left(\pi_{S(s)}(s)\right)^{i}=0$, with $a_{i} \in A_{X}$ and $a_{0} \neq 0$ (since $\pi_{S(s)}(s)$ is a non-zero-divisor). Define then

$$
t=a_{0} \mathbf{1}_{n}+\left(s^{n}+\sum_{i=1}^{n-1} a_{i} s^{i}\right) \in S_{W}^{n} .
$$

This element has support $S(t)=I-S(s)$.

Because of the multiplicative property of supports, $S\left(s_{1} s_{2}\right)=S\left(s_{1}\right) \cap S\left(s_{2}\right)$, we obtain $r$ minimal subsets $S_{a}, 1 \leqq a \leqq r \leqq k$ of $I=\{1,2, \ldots, k\}$ such that

(1) $S_{a} \cap S_{b}=\emptyset$ if $a \neq b$;

(2) $I=\bigcup S_{a}$;

(3) $\exists z_{a} \in S_{W}^{n}$ with $S\left(z_{a}\right)=S_{a}$;

(4) if for some $z \neq 0 \in S_{\bar{W}}^{n}$ we have $S(z) \subset S_{a}$ then $S(z)=S_{a}$.

Consider the projection:

$$
\pi_{S_{a}}: S_{W}^{n} \rightarrow \bigoplus_{j \in S_{a}} \pi_{j}\left(S_{\frac{W}{n}}^{n}\right)
$$

The image is (by (4)) an integral domain and the kernel $\mathfrak{p}_{a}=\operatorname{ker} \pi_{S_{a}}$ is a prime ideal. In fact this is a minimal prime ideal: suppose that $p \subset \mathfrak{p}_{a}$ is a prime ideal and let $x \in \mathfrak{p}_{a}$; then by Lemma 6.4.1 there exists a $z \in S_{W}^{n}$ with $S(z)=S_{a}$ and $z x=0$; now $z \bmod \mathfrak{p}$ is not zero and hence $x \bmod \mathfrak{p}$ must be zero for otherwise there would be a zero-divisor in the integral domain $S_{W}^{n} / \mathfrak{p}$; this means that $\mathfrak{p}=\mathfrak{p}_{a}$ and $\mathfrak{p}_{a}$ is indeed minimal. So in this case $\operatorname{Spec}\left(S_{W}^{n}\right)$ has $r$ irreducible components isomorphic to $\operatorname{Spec}\left(S_{W}^{n} / \mathfrak{p}_{a}\right)$.

For example, consider an elliptic curve $X$ with affine coordinate ring $A_{X}$ given in Eq. (2.2.3). Take $g=\left(\begin{array}{cc}1 & 0 \\ \lambda^{-2 k} & 1\end{array}\right) \in G l\left(2, H_{\lambda}\right)$ and the free $A_{X}$-module $W=g \cdot H_{X}^{(2, \mathrm{alg})} \in G r_{X}^{2}$. Then $s=\left(\begin{array}{cc}\lambda^{2 k} & 0 \\ 0 & -\lambda^{2 k}\end{array}\right)$ is an element of the stabilizer subalgebra $S_{W}^{1,1}$ of type $(1,1)$, corresponding to the "homogeneous" Heisenberg algebra 
of $g l\left(2, H_{\lambda}\right)$, since $g^{-1} \operatorname{sg} \in g l\left(2, A_{X}\right)$. But $\lambda^{2 k} \in A_{X}$ and $\mathbf{1} \in S_{W}^{1,1}$ so also the zerodivisors $\left(\begin{array}{cc}\lambda^{2 k} & 0 \\ 0 & 0\end{array}\right)$ and $\left(\begin{array}{cc}0 & 0 \\ 0 & \lambda^{2 k}\end{array}\right)$ belong to $S_{W}^{1}{ }^{1}$. In fact $S_{W}^{1}{ }^{1} \simeq A_{X}\left(\begin{array}{cc}\lambda^{2 k} & 0 \\ 0 & 0\end{array}\right)$ $\oplus A_{X}\left(\begin{array}{cc}0 & 0 \\ 0 & \lambda^{2 k}\end{array}\right)$ is the direct sum of two copies of $A_{X}$ and its spectrum consists of two copies of the affine curve $X-p$ touching to order $k$ at the points over the origin of the Riemann sphere. Of course the method of Sect. 5 provides us with examples of non-principal stabilizer algebras which are irreducible.

It can happen that $S_{W}^{n}$ is the Cartesian product of $\kappa>1 \mathbb{C}$-algebras. In this case $\operatorname{Spec}\left(S_{\bar{W}}^{n}\right)$ is the disjoint union of $\kappa$ components and $S_{W}^{n}$ will contain $\kappa$ nontrivial idempotents; an idempotent is an element $e \in S_{\bar{W}}^{n}$ such that $e \neq 0,1$ and $e^{2}=e$. Idempotents are zero-divisors that are independent of $\lambda$. Now since the restriction of $e$ to any of the diagonal blocks is not a zero-divisor we must have

$$
e=\sum_{i} e_{i} \mathbf{1}_{n_{i}}
$$

where $e_{i}=0$ or $1, \mathbf{1}_{n_{i}}$ is the identity matrix in the $n_{i}^{\text {th }}$ diagonal block of $H^{n}$ and not all of the $e_{i}$ 's are simultaneously 0 or 1 . The fact that $S_{\bar{W}}^{n}$ contains an idempotent means that $W$ is a direct sum

$$
W=e W \oplus(1-e) W
$$

and that the summands are embeddings of elements of lower rank Grassmannians $G r_{X}^{p}$ and $G r_{X}^{q}$. In other words, there is an element $g \in g l\left(n, H_{\lambda}\right)$ such that $W=g H_{X}^{(n, \text { hol })}$ and such that $g$ is the direct sum of two elements, $e g$ and $(1-e) g$, of lower dimensional loop groups $g l\left(p, H_{\lambda}\right)$ and $g l\left(q, H_{\lambda}\right)$ (where $p=\sum_{i=1}^{k} e_{i} n_{i}$, $\left.q=\sum_{i=1}^{k}\left(1-e_{i}\right) n_{i}\right)$.

6.5. The total quotient ring $Q\left(S_{\bar{W}}^{n}\right)$ is the ring of fractions $\left(S^{-1}\right)\left(S_{\bar{W}}^{n}\right)$, where $S$ is the multiplicative set of non-zero-divisors in $S_{\bar{W}}^{n}$. As is well known (see e.g., $[\mathrm{Ku}]$ ) this is the Cartesian product of the fields $F^{i}=Q\left(S_{\bar{W}}^{n} / \mathfrak{p}_{i}\right)$ of rational functions on the irreducible components $\operatorname{Spec}\left(S_{\bar{W}}^{n} / \mathfrak{p}_{i}\right)$. Another convenient description of this ring of rational functions is given by the following

Lemma 6.5.1. $Q\left(S_{\bar{W}}^{n}\right)=K_{X} \otimes_{A_{X}} S_{\bar{W}}^{n}$.

Proof. Recall that if $B, C$ are rings, $S$ a multiplicative subset in $B$ and $\phi: B \rightarrow C$ a homomorphism such that:

(1) For all $s \in S, \phi(s)$ is invertible in $C$;

(2) If $\phi(b)=0$ then there exists an $s \in S$ such that $s b=0$;

(3) Every element of $C$ can be written as $\phi(b) \phi(s)^{-1}$, for $b \in B, s \in S$, then $C \simeq S^{-1} B$. We check these conditions for the homomorphism $\psi: S_{\bar{W}}^{n} \rightarrow K_{X} \otimes_{A_{X}} S_{\bar{W}}^{n}, s \mapsto 1 \otimes s$ and the multiplicative set of non-zerodivisors in $S_{W}^{n}$.

Since $S_{\bar{W}}^{n}$ is an integral extension of $A_{X}$ also $K_{X} \otimes_{A_{X}} S_{\bar{W}}^{n}$ is an integral extension of $K_{X}$. Let $s \in S$. Then $\psi(s)$ satisfies a monic equation $\psi(s)^{n}+k_{n-1} \psi(s)^{n-1}$ $+\cdots+k_{0}=0, k_{i} \in K_{X}$. Since for $s \in S$ also $\psi(s)$ is a non-zero-divisor we may assume that $k_{0} \neq 0$ and we see that $\psi(s)$ is invertible (since $\psi(s)^{-1}=-\frac{1}{k_{0}}\left(\psi(s)^{n-1}\right.$ $\left.\left.+k_{n-1} \psi(s)^{n-2}+\cdots+k_{1}\right)\right)$, verifying property (1). 
Next suppose that $\psi(t)=0$, and $t \in S_{\bar{W}}^{n}$. Then there exists an $a \in A_{X}$ such that $a t=0$. But $S_{\bar{W}}^{n}$ is torsion free as $A_{X}$-module and hence $t=0$. This proves part (2).

For part (3) let $Y=\sum \frac{\alpha_{i}}{\beta_{i}} \otimes s_{i} \in K_{X} \otimes S_{W}^{n}$. Then $Y=\sum \frac{\alpha_{i} B_{i}}{\beta_{1} \ldots \beta_{t}} \otimes s_{i}$, where $B_{i}=\prod_{i \neq j} \beta_{j}$ and we find $Y=\frac{1}{\beta_{1} \ldots \beta_{t}} \otimes \sum \alpha_{i} B_{i} s_{i}=\psi\left(\sum \alpha_{i} B_{i} s_{i}\right) \psi\left(\beta_{1} \ldots \beta_{t}\right)^{-1}$, and also point (3) is verified.

So $K_{X} \otimes S_{\bar{W}}^{n}=F_{1} \times F_{2} \times \ldots F_{r}$, where $F_{a}$ is the function field of the $a^{\text {th }}$ irreducible component $\operatorname{Spec}\left(S_{W}^{n} / \mathfrak{p}_{a}\right)$ of $\operatorname{Spec}\left(S_{W}^{n}\right)$. The support of $F_{a}$ is of course the subset $S_{a} \subset I=\{1,2, \ldots, k\}$ introduced in Sect. 6.4 to determine the minimal primes of $S_{\frac{n}{W}}$.

6.6. In the previous subsection we saw that we could determine the irreducible components of $\operatorname{Spec}\left(S_{W}^{n}\right)$ by studying the decomposition into fields of the tensor product $K_{X} \oplus S_{\bar{W}}^{n}$. By tensoring with the larger field $L_{\lambda}$ of Laurent series we get even more detailed information about $\operatorname{Spec}\left(S_{W}^{n}\right)$ : we will use the field decomposition of the tensor product $L_{\lambda} \otimes S_{W}^{n}$ to define a filtration on $S_{W}^{n}$ which allows us (in the next chapter) to compactify $\operatorname{Spec}\left(S_{W}^{n}\right)$ to a projective curve $Y$ with a natural covering map $Y \rightarrow X$.

Each field $F_{a}$ occurring in $K_{X} \oplus S_{W}^{n}$ will decompose in general into more fields after tensoring with $L_{\lambda}: L_{\lambda} \otimes F_{a}=F_{a}^{1} \times F_{a}^{2} \times \ldots \times F_{a}^{k_{a}}$. (The number of fields $k_{a}$ occurring in this decomposition will turn out to be the number of points lying over $p \in X$ in the irreducible component of the compactification $Y$ of $\operatorname{Spec}\left(S_{\bar{W}}^{n}\right)$ with function field $F_{a}$.) The number of fields $\sum k_{a}$ in $L_{\lambda} \otimes S_{W}^{n}$ is at most $k$, the number of blocks in the Heisenberg algebra.

Let us denote by $S_{a}^{e}$ the support of the field $F_{a}^{e}$. Let $f \in F_{a}^{e}$ and $i \in S_{a}^{e}$. If $\pi_{i}(f)$ is the zero of a polynomial $P(t) \in L_{\lambda}[t]$, then the $i^{\text {th }}$ diagonal block of $P(f) \in F_{a}^{e}$ is the zero matrix. Hence $P(f)$ is identically zero and all projections $\pi_{i}(f), i \in S_{a}^{e}$ satisfy the same equations. This means that all projections $\pi_{i}\left(F_{a}^{e}\right)$ are isomorphic field extensions of $L_{\lambda}$ of degree $\ell_{a}^{e}=\left[F_{a}^{e}: L_{\lambda}\right]$. Each $\pi_{i}\left(F_{a}^{e}\right)$ is a subfield of $L_{\lambda}\left[P_{i}\right]$.

First we state some general facts about $L_{\lambda}\left[P_{n}\right] . L_{\lambda}\left[P_{n}\right]$ is a finite field extension of $L_{\lambda}$ with $\left[L_{\lambda}\left[P_{n}\right]: L_{\lambda}\right]=n$ (since $P_{n}^{n}=\lambda$ ). In fact $L_{\lambda}\left[P_{n}\right]$ is a Galois extension of $L_{\lambda}$ with Galois group $\mathbb{Z}_{n}=\left\{1, \omega, \omega^{2}, \ldots, \omega^{n-1}\right\}$, where $\omega$ is a primitive $n^{\text {th }}$ root of unity. This group acts by multiplication $P_{n} \mapsto \omega^{i} P_{n}, i=0,1, \ldots, n-1$ on the generator of the extension. By Galois theory the only subfield extensions of $L_{\lambda}\left[P_{n}\right]$ are the fixed fields of subgroups $H$ of $\mathbb{Z}_{n}$. These fields are of the form $L_{\lambda}\left[P_{n}^{m}\right]$, $m=\frac{n}{\ell}$, corresponding to the subgroups $\mathbb{Z}_{\ell}$ of $\mathbb{Z}_{n}$, where $\ell$ runs over the divisors of $n$. In particular this means that the minimal polynomial of an element $X$ of $L_{\lambda}\left[P_{n}\right]$ (generating some subfield $L_{\lambda}[X]$ of $L_{\lambda}\left[P_{n}\right]$ ) must have degree $\ell$ dividing $n$, and in this case $X \in L_{\lambda}\left[P_{n}^{m}\right], m=\frac{n}{\ell}$.

Hence if $i$ is in the support $S_{a}^{e}$ of the field $F_{a}^{e}$ and $\ell_{a}^{e}$ is the degree $\left[F_{a}^{e}: L_{\lambda}\right]$ of the extension (equivalently $\ell_{a}^{e}$ is the rank of $\pi_{i}\left(S_{W}^{n}\right)$ ), then $\ell_{a}^{e}$ divides $n_{i}$, the size of the $i^{\text {th }}$ block: $n_{i}=m_{i} \ell_{a}^{e}$ and we have:

$$
S_{W}^{n} \subset \bigoplus_{i=1}^{k} L_{\lambda}\left[P_{n_{l}}^{m_{l}}\right]
$$


We then define on $S_{\bar{W}}^{n}$ the filtration $\mathfrak{o}_{n, \ell}$, see (5.6.6), induced by the inclusion (6.6.1) where $\underline{\ell}$ is the collection of integers $\bar{\ell}_{i}=n_{i} / m_{i}$. Note that for all $i, j \in S_{a}^{e}, s \in S_{\bar{W}}^{n}$ we have $\mathfrak{o}_{n_{i}, \ell_{i}}\left(\pi_{i}(s)\right)=\mathfrak{o}_{n_{j}, \ell_{j}}\left(\pi_{j}(s)\right)$. In other words the blocks within the support of a single field component contribute in the same way to the order of $s$. If each of the fields $F_{a}^{e}$ has support in a single block, (i.e., if there are exactly $k$ fields in $L_{\lambda} \otimes S_{\bar{W}}^{n}$ ), $\underline{l}$ is a partition of the rank $\ell$ of $S_{\bar{W}}^{n}$ over $A_{X}$.

Proposition 6.6.1. Let $S_{W}^{n}=S_{W}^{\text {fin }} \cap \mathscr{H}^{n}$ be the stabilizer algebra of type $\underline{n}$ $=n_{1}+n_{2}+\cdots+n_{k}$ of $W \in G r_{X}^{n}$. If $k_{0} \leqq k$ is the number of fields in $L_{\lambda} \otimes S_{\bar{W}}^{n}$ and $\left(S_{\bar{W}}^{n}\right)_{i}$, denotes the elements of order at most $i$ in $S_{\bar{W}}^{n}$, then

$$
\left(S_{\bar{W}}^{n}\right)_{i+1} /\left(S_{\bar{W}}^{n}\right)_{i} \simeq \mathbb{C}^{k_{0}},
$$

for all $i$ larger than some $i_{0}$.

Proof. We first study the simplest case: a principal stabilizer algebra $S_{W}^{n}$ of rank $n$ over $A_{X}$. Let $\left\{s_{1}, s_{2}, \ldots, s_{n}\right\}$ be a maximal independent set in $S_{W}^{n}$. Then $\left\{s_{1}, s_{2}, \ldots, s_{n}\right\}$ is also a basis for $L_{\lambda} \otimes S_{W}^{n}=L_{\lambda}\left[P_{n}\right]$ (or rather the elements $1 \otimes s_{i}$ are, but we will not make this distinction). Hence in particular we can write

$$
P_{n}=\sum_{i=1}^{n} a_{i}(\lambda) s_{i}, \quad a_{i}(\lambda) \in L_{\lambda} .
$$

We can split each of the Laurent series $a_{i}(\lambda)$ into a Laurent polynomial $\beta_{i}(\lambda) \in \mathbb{C}\left[\lambda, \lambda^{-1}\right]$ and another Laurent series $\gamma_{i}(\lambda)$ such that $a_{i}(\lambda)=\beta_{i}(\lambda)+\gamma_{i}(\lambda)$, and $\mathfrak{o}_{n}\left(\beta_{i}(\lambda) s_{i}\right) \geqq 1, \mathfrak{o}_{n}\left(\gamma_{i}(\lambda) s_{i}\right)<1$. Then we have, recalling that $P_{n}$ has order 1 :

$$
\sum_{i=1}^{n} \beta_{i}(\lambda) s_{i}=P_{n}+\text { lower order terms . }
$$

Now every Laurent polynomial $\beta_{i}(\lambda)$, when multiplied by any sufficiently high power of $\lambda$, is the highest order part of an element $f_{i}(\lambda) \in A_{X}: f_{i}(\lambda)=\beta_{i}(\lambda) \lambda^{m}+$ lower order terms. We fix an $m_{0}$ that works for all $\beta_{i}(\lambda)$ and find:

$$
\sigma:=\sum_{i=1}^{n} f_{i}(\lambda) s_{i}=\lambda^{m_{0}} P_{n}+\text { lower order terms . }
$$

Now, as $f_{i} \in A_{X}$ and $s_{i} \in S_{W}^{n}$, we have $\sigma \in S_{W}^{n}$ and we have found an element of order $1(\bmod n)$. The first $n$ powers of $\sigma$ are then independent and, remembering that multiplication by $\lambda$ increases the order of an element by $n$, we see that there is at least one element of every sufficiently high order in $S_{W}^{n}$. Since $S_{W}^{n} \subset L_{\lambda}\left[P_{n}\right]$ there is exactly one independent element of each high enough order (up to addition of lower order elements) and we find $\left(S_{W}^{n}\right)_{i+1} /\left(S_{W}^{n}\right)_{i}=\mathbb{C}$ for large $i$.

The general case is essentially the same: for every field $F_{a}^{e}$ in $L_{\lambda} \otimes S_{\bar{W}}^{n}$, with support $S_{a}^{e}$ and of dimension $\ell_{a}^{e}$ over $L_{\lambda}$, one shows that there is an element $\sigma_{a}^{e} \in S_{W}^{n}$ which has its highest order term in the blocks indexed by $i \in S_{a}^{e}$ and with order $\mathfrak{o}_{\underline{n}, \ell}\left(\sigma_{a}^{e}\right)=1\left(\bmod \ell_{a}^{e}\right)$. The first $\ell_{a}^{e}$ powers of $\sigma_{a}^{e}$ are independent and since multiplying by $\lambda$ increases the order in this block $\ell_{a}^{e}$, we find again an element of every high enough order in $S_{W}^{n}$. Since such elements $\sigma_{a}^{e}, \sigma_{a^{\prime}}^{e^{\prime}}$ (and their first $\ell_{a}^{e}$, respectively $\ell_{a^{\prime}}^{e^{\prime}}$ powers) for distinct subfields $F_{a}^{e}$ and $F_{a^{\prime}}^{e^{\prime}}$ are linearly independent we find for every field in $L_{\lambda} \otimes S_{\bar{W}}^{n}$ one independent element and $S_{\bar{W}}^{n}$ contains $k_{0}$ independent elements of all high enough orders. 
To finish this discussion of the filtration of $S_{\bar{W}}^{n}$ we note that $\left(S_{\bar{W}}^{n}\right)_{0}$ is isomorphic to $\mathbb{C}^{k}$ if $S_{W}^{n}$ is the Cartesian product of $\kappa \mathbb{C}$-algebras (see the discussion at the end of Sect. 6.4) and that

$$
\left(S_{\bar{W}}^{n}\right)_{i}=0, \quad i<0 .
$$

To prove this last statement we use the natural filtration of the $n$-component Laurent series $L_{\lambda}^{n}$ given by $\mathfrak{o}(s)=\max _{i=0}^{n-1}\left(s_{i}(\lambda)\right)$ if $s=\left(s_{0}(\lambda), \ldots, s_{n-1}(\lambda)\right) \in L_{\lambda}^{n}$. This induces a filtration on the subspaces $W \in G r_{X}^{n}$ which has the following interpretation: if $W$ corresponds to a bundle $E$ and $w \in W$ has order $k$ then $w$ corresponds to a section $\varepsilon$ of $\mathscr{E}$ which has a pole of order $k$ at $p$ and is regular elsewhere. If $k$ is negative then $\varepsilon$ has a zero and is a global section of $\mathscr{E}$. Since the space of global sections is finite dimensional there can occur only a finite number of negative orders for elements of a fixed $W$. Consider then an element $s \in S_{W}^{n}$ and $w \in W$ such that $s \cdot w \neq 0$. One easily chekcs that if the order of $s$ were negative the order of $s \cdot w$ would be strictly less than the order of $w$. But this would imply, since $s W \subset W$, that in the set $\left\{s^{i} \cdot w\right\} \subset W$ an infinite number of elements of distinct negative order occur, which is impossible. This proves (6.6.6).

\section{The Compactification of $\operatorname{Spec}\left(S_{\bar{W}}^{n}\right)$}

7.1. Next we want to compactify the affine curve $\operatorname{Spec}\left(S_{\bar{W}}^{n}\right)$. This uses the filtration on $S_{\bar{W}}^{n}$ described in Sect. 6.6. Let us first recall some elementary facts about filtered and graded rings and homogeneous spectra (see e.g. Hartshorne [Ha]).

Let $A$ be a ring with some order function $\mathfrak{D}$ and corresponding filtration $A_{0} \subset A_{1} \subset A_{2} \subset \ldots$, (so $A_{-1}$ is zero). Define an associated graded ring

$$
R(A)=\bigoplus_{i=0}^{\infty} R_{i},
$$

where $R_{i}=A_{i}$. To simplify notation we will write usually $R$ if it is clear to which filtered ring $A$ we refer. Let $w$ denote the element of degree 1 in $R$ which is the image of $1 \in A$ under the embedding of $A_{0}$ in $A_{1}$. So if $\alpha$ belongs to $A_{i}$ we obtain an element $\alpha^{(i)} \in R_{i}$ and we have $\alpha^{(j)}=\alpha^{(i)} w^{j-i}$ if $j \geqq i$.

Define for arbitrary homogeneous element $f \in R$ the homogeneous ring of quotients of $R$ by $R_{(f)}=\left\{g / f^{n} \mid \operatorname{deg}(g)=\operatorname{deg}\left(f^{n}\right), n \geqq 0\right\}$. Then one can recover the original ring $A$ and its filtration from the pair $(R, w)$ : we have $A \cong R_{(w)}$, via $\alpha \in A_{i} \mapsto \alpha^{(i)} / w^{i} \in R_{(w)}$.

Consider next the homogeneous spectrum of $R$. Let $R_{+}=\bigoplus_{i=1}^{\infty} R_{i}$ and define

$$
\operatorname{Proj}(R)=\left\{p \in \operatorname{Spec}(R) \mid p \text { is homogeneous and } R_{+} \notin p\right\} \text {. }
$$

Recall that an ideal $I$ of a graded ring is called homogeneous if $I=\oplus I \cap R_{i}$ or equivalently if it is generated by homogeneous elements. $\operatorname{Proj}(R)$ is a topological space with basic open sets

$$
D_{+}(f)=\{p \in \operatorname{Proj}(R) \mid f \notin p\},
$$

for $f$ a homogeneous element of $R$. Just as $\operatorname{Spec}(A)$ also $X=\operatorname{Proj}(R)$ has its sheaf of local rings, again denoted by $\mathcal{O}_{X}$ and called the structure sheaf. The space of sections of the structure sheaf over the basic open set $D_{+}(f)$ is the $\operatorname{ring} R_{(f)}$ and the stalk at a point $p \in X$ is the local ring $\mathcal{O}_{X, p}=R_{(p)}$, the degree zero part of the ring of 
quotients of $R$ with denominator set the homogeneous elements of $R$ not in $p$. Closed sets of $\operatorname{Proj}(R)$ are the components of $D_{+}(f)$ or more generally of the form $V(I)=\{p \in \operatorname{Proj}(R) \mid I \subset p\}$ for homogeneous ideals $I$.

In contrast to an open subset $D_{+}(f)$ (which is isomorphic to the affine scheme $\left.\operatorname{Spec}\left(R_{(f)}\right)\right)$, the closed sets $V(I)$ don't have a canonical scheme structure. However, note that $V\left(I^{n}\right)=V(I), n>0$ and that we have an isomorphism of topological spaces

$$
\phi_{n}: \operatorname{Proj}\left(R / I^{n}\right) \rightarrow V\left(I^{n}\right), \quad q \in \operatorname{Proj}\left(R / I^{n}\right) \mapsto \psi_{n}^{-1}(q),
$$

where $\psi_{n}: R \rightarrow R / I^{n}$ is the canonical surjection.

Letting $Y_{n}=\operatorname{Proj}\left(R / I^{n}\right)$ and $\mathcal{O}_{Y_{n}}$ its structure sheaf we have a closed immersion

$$
\left(\phi_{n}, \phi_{n}^{\#}\right):\left(Y_{n}, \mathcal{O}_{Y_{n}}\right) \rightarrow\left(X, \mathcal{O}_{X}\right) \text {. }
$$

This means that the map $\phi_{n}^{\#}: \mathcal{O}_{X} \rightarrow \phi_{n *} \mathcal{O}_{Y}$ is surjective, where $\phi_{n}^{\#}$ is, on the level of stalks, the canonical map $R_{\left(\phi_{n}(q)\right)} \rightarrow\left(R / I^{n}\right)_{(q)}$. Hence each closed immersion defines a sheaf of ideals $\mathfrak{I}^{n}$, the kernel of $\phi^{\#}$, and $\mathcal{O}_{X} / \mathfrak{I}^{n}$ is a sheaf with support on $V(I)$. We define the $n^{\text {th }}$ infinitesimal neighborhood of $V(I)$ as the scheme $\left(V(I), \mathcal{O}_{X} / \mathfrak{J}^{n}\right)$; it carries information about the way $V(I)$ is embedded in $X$. It is then natural to consider the inverse limit of these scheme structures on $V(I)$. The locally ringed space $\hat{X}=\left(V(I), \mathcal{O}_{\hat{X}}\right)$ with $\mathcal{O}_{\hat{X}}=\lim \mathcal{O}_{X} / \mathfrak{I}^{n}$ is called the formal completion of $X$ along the subscheme $V(I)$. The formal completion is independent of the ideal we use to define the subset $V(I)$.

Lemma 7.1.1. Let $R$ be a Noetherian graded ring and $I$ a homogeneous ideal of $R$ such that $V(I)$ contains only a finite number of maximal ideals $p_{1}, p_{2}, \ldots, p_{k}$. Then the structure sheaf $\mathcal{O}_{\hat{X}}$ of the formal completion of $X=\operatorname{Proj}(R)$ along $V(I)$ has a stalk at $p_{i}$ isomorphic to the $m_{p_{t}}$-adic completion $\hat{\mathcal{O}}_{X, p_{t}}$ of the local ring $\mathcal{O}_{X, p_{t}}$, where $m_{p_{i}}$ is the maximal ideal of $\mathcal{O}_{X, p_{i}}$.

Proof. Without loss of generality we may assume that $I$ is a radical ideal; then we have $I=\bigcap p_{i}$. Using the fact that $R$ is Noetherian and hence the ideals $p_{i}$ are finitely generated one checks that also $I^{n}=\bigcap p_{i}^{n}$. Now the ideals $p_{i}^{n}, p_{j}^{n}$ are coprime if $i \neq j$ and hence by the Chinese remainder theorem we have $R / I^{n}=\bigoplus R / p_{i}^{n}$. Hence we also have $\hat{R}=\lim R / I^{n}=\bigoplus \lim R / p_{i}^{n}$. The ring $\hat{R}$ determines the formal completion sheaf $\mathcal{O}_{\hat{X}}$ : we have $\mathcal{O}_{\hat{X}, p_{i}}=\hat{R}_{\left(\hat{p}_{i}\right)}$, where $\hat{p}_{i}=\lim p_{i} / I^{n}$. But

$$
\hat{R}_{\left(\hat{p}_{t}\right)}=\underset{\lim }{\longleftarrow} R_{\left(p_{i}\right)} / m_{p_{t}}^{n}=\longleftarrow \mathcal{H}_{X, p_{t}} / m_{p_{t}}^{n}=\hat{\mathcal{O}}_{X, p_{t}} .
$$

If $R$ is a finitely generated graded $\mathbb{C}$-algebra with $\operatorname{Proj}(R)$ isomorphic to the Proj of a quotient $S$ of the polynomial algebra $\mathbb{C}\left[t_{0}, \ldots, t_{N}\right]$ with $\operatorname{deg}\left(t_{i}\right)=1$ we call $\operatorname{Proj}(R)$ and also $R$ projective, since in that case $\operatorname{Proj}(R)$ is isomorphic to a closed subscheme in the projective space $\mathbb{P}^{N}$.

Let us return to the case where we have a filtered ring $A$, an associated graded ring $R(A)$. Then we have

$$
\operatorname{Proj}(R)=D_{+}(w) \cup V(w) .
$$

Now $D_{+}(w) \simeq \operatorname{Spec}\left(R_{(w)}\right) \simeq \operatorname{Spec}(A)$, so we see that $V(I)$ is "the divisor at infinity" that has to be added to $\operatorname{Spec}(A)$ to obtain the projective scheme $\operatorname{Proj}(R)$. We have seen that each of the rings $R / w^{n} R$ determines, via $\operatorname{Proj}\left(R / w^{n} R\right)$, a closed subscheme structure on $V(w)$. 
We will be interested in the case where $X=\operatorname{Proj}(R)$ is projective of dimension 1 and $V(w)$ contains a finite number of closed points. Then Lemma 7.1.1 tells us in particular that the points in $V(w)$ that we have to add to complete $\operatorname{Spec}(A)$ are all nonsingular if and only if the stalks of the formal completion are isomorphic to the ring of formal power series in $\operatorname{dim}(X)=1$ variables.

7.2. We can first of all apply this to the ring $A_{X}$ of Sect. 2.1. This ring is filtered by the order of the pole at $p$. It will be no surprise that the Proj of $R\left(A_{X}\right)$ is nothing but a projective nonsingular integral scheme isomorphic to $X$ itself and that the Proj of $R / w^{n} R$ can be identified with the $n^{\text {th }}$ infinitesimal neighborhood of $p$, the missing point which has to be added to the space $\operatorname{Spec}\left(A_{X}\right) \cong X-p$ to get $X$ back.

This can be seen as follows. $A_{X}$ is a subalgebra of $L_{\lambda}=\mathbb{C}[\lambda] \oplus \mathbb{C}\left\{\lambda^{-1}\right\} / \lambda$ and for every $i \geqq i_{0}$, for some $i_{0}$, there are normalized elements

$$
f_{i}=\sum_{j \geqq 0} c_{j} \lambda^{i-j}, \quad c_{0}=1
$$

of order $i$. Then the graded ring $R=R\left(A_{X}\right)$ is a subalgebra of $L_{\bar{\lambda}, w^{-1}}=L_{\bar{\lambda}} \otimes L_{w^{-1}}$ generated by a subset of the elements of $R$ of the form

$$
f_{i}^{(i)}=\sum_{j \geqq 0} c_{j} \bar{\lambda}^{i-j} w^{j}
$$

corresponding to a set of generators for $A_{X}$. (So one can identify $\lambda$ with $\bar{\lambda} / w$.) One checks that $R$ is a quotient of a quasi-homogeneous polynomial algebra and that $\operatorname{Proj}(R)$ is projective and hence complete.

Next we investigate $V(w)$, the divisor at infinity that we have to add to make $\operatorname{Spec}\left(A_{X}\right)$ complete in $X^{\prime}=\operatorname{Proj}(R)$. Let us for simplicity first consider the closed subscheme structure on $V(w)$ determined by $R / w R$. The normalization of the $f_{i}$ in (7.2.1) is such that $f_{i} f_{j}=\lambda^{i+j}+$ lower order for all $i, j \geqq i_{0}$. These elements $f_{i}$ of $A_{X}$ give elements $f_{i}^{(i)}$ of $R$ satisfying $f_{i}^{(i)} f_{j}^{(j)}=\bar{\lambda}^{i+j}(\bmod w R)$. This allows us to define a map

$$
\phi: R / w R \rightarrow C[t], \quad \operatorname{deg}(t)=1,
$$

by setting the restriction $\phi_{j}$ to $(R / w R)_{j}$ for $j<i_{0}$ equal to zero and defining $\phi_{i}\left(\bar{f}_{i}^{(i)}\right)=t^{i}$ (and extending linearly). So $t$ can be identified with $\bar{\lambda}(\bmod w R)$. Then $\phi$ is a graded ring homomorphism and $\phi_{i}, i \geqq i_{0}$ is the isomorphism. In general if $\phi: R \rightarrow S$ is a homomorphism of graded rings such that the restriction $\phi_{n}: R_{n} \rightarrow S_{n}$ is an isomorphism for all $n$ large enough, then we have $\operatorname{Proj}(R) \simeq \operatorname{Proj}(S)$. We call $R$ and $S$ isomorphic in large degree in this case. So in our case, where $R=R\left(A_{X}\right)$, the quotient $R / w R$ is isomorphic in large degree to the polynomial ring in one variable and one sees that $\operatorname{Proj}(R / w R)$ consists of one point $p$ (corresponding to the zero ideal in $\mathbb{C}[t])$. Hence $\operatorname{Proj}\left(R\left(A_{X}\right)\right)$ is obtained from $\operatorname{Spec}(A)$ by adding a single point $p$.

To investigate whether this added point is nonsingular or not we study the formal completion of $\operatorname{Proj}(R)$ along the point $V(w)$. The structure sheaf of this formal completion is obtained from the inverse limit of the rings $R / w^{n} R$. Now for $i \geqq i_{0}+n$ we have $R_{i} / w^{n} R_{i-n}=\mathbb{C} \bar{\lambda}^{i} \oplus \mathbb{C} w \bar{\lambda}^{i-1} \oplus \cdots \oplus w^{n-1} \bar{\lambda}^{i+1-n}\left(\bmod w^{n} R\right)$. Hence the ring $R / w^{n} R$ is isomorphic in large degree to $\mathbb{C}[w, \bar{\lambda}] /\left\langle w^{n}\right\rangle$, The stalk of the structure sheaf of $\operatorname{Proj}\left(R / w^{n} R\right)$ at the unique maximal ideal is isomorphic to the localization of $\mathbb{C}[w, \bar{\lambda}] /\left\langle w^{n}\right\rangle$ at $\langle w\rangle$ which is easily calculated to be $\mathbb{C}[\mu] / \mu^{n}$, where $\mu=w / \bar{\lambda}=1 / \lambda$ is a local coordinate at $p \in V(w)$. Hence the stalk of the formal 
completion is $\mathbb{C} \llbracket \mu \rrbracket$ and the added point is indeed nonsingular. Since $\operatorname{Proj}(R)=\operatorname{Spec}\left(A_{X}\right) \cup V(w)$ and $A_{X}$ is the coordinate ring of a nonsingular curve we see that all points of $\operatorname{Proj}(R)$ are nonsingular and $X^{\prime}=\operatorname{Proj}(R)$ is a nonsingular reduced curve with $\operatorname{Spec}\left(A_{X}\right)$ as open subscheme. In particular $X$ and $X^{\prime}$ have the same function field. By the uniqueness, up to isomorphism, of a nonsingular irreducible projective curve with given function field we have then $\operatorname{Proj}(R)=X$, and we have recovered our complete curve from the affine coordinate ring together with its filtration and the local coordinate $\mu=1 / \lambda$ at $p$.

7.3. Next we consider the stabilizer algebra $S_{W}^{n}$ for $W \in G r_{X}^{n}$ and $\underline{n}$ a partition into $k$ parts. Let $R=R\left(S_{\bar{W}}^{n}\right)$. Then, since $S_{\bar{W}}^{n}$ is finitely generated, as before $R$ is a quotient of a quasi-homogeneous polynomial ring and hence $\operatorname{Proj}(R)$ is a projective complete curve. We have $\operatorname{Proj}(R)=\operatorname{Spec}\left(S_{\bar{W}}^{n}\right) \cup V(w)$. Similar to the discussion for $A_{X}$ we introduce normalized elements in $S_{W}^{n}$. To that end let $S_{a}^{e}$ be the support of the field $F_{a}^{e}$ in the decomposition of $L_{\lambda} \otimes S_{\bar{W}}^{n}$ and define

$$
\lambda_{a}^{e}=\sum_{i \in S_{a}^{e}} \mathscr{P}_{n_{t}}^{m_{i}} \in \mathscr{H}^{n},
$$

where $n_{i} / m_{i}$ is the degree of the field extension $F_{a}^{e}$ over $L_{\lambda}$. Note that $\lambda_{a}^{e}$ is an element of order 1 in the filtration $\mathfrak{o}_{n, \ell}$, see Sect. 5.6. Then we require that the highest order term of the normalized element $\sigma_{a, i}^{e} \in S_{W}^{n}$ of order $i$ with highest order term in the blocks indexed by $S_{a}^{e}$ is precisely $\left(\lambda_{a}^{e}\right)^{i}$. (It is easy to check that we can indeed normalize elements of $S_{\bar{W}}^{n}$ in this way.)

Then we have

$$
\sigma_{a, i}^{e} \sigma_{b, j}^{f}=\delta_{e, f} \delta_{a, b}\left(\lambda_{a}^{e}\right)^{i+j}+\text { lower order, } \quad i, j \geqq i_{0} .
$$

We find in the same way as before that $R / w^{n} R \simeq \prod \mathbb{C}\left[w, \bar{\lambda}_{a}^{e}\right] / w^{n}$ (isomorphism in large degree), where $\bar{\lambda}_{a}^{e} / w=\lambda_{a}^{e}$ and the product is taken over the indices describing the $k_{0}$ fields in $L_{\lambda} \otimes S_{\bar{W}}^{n}$. Hence the $k_{0}$ stalks of the formal completion of $\operatorname{Proj}(R)$ along $V(w)$ are isomorphic to the formal power series rings $\mathbb{C} \llbracket \mu_{a}^{e} \rrbracket$, where $\mu_{a}^{e}=w / \lambda_{a}^{e}=\left(\lambda_{a}^{e}\right)^{-1}$. Hence $V(w)$ consists of exactly $k_{0}$ nonsingular points $p_{a}^{e}$ that have to be added to $\operatorname{Spec}\left(S_{\bar{W}}^{n}\right)$ to obtain the reduced complete curve $Y=Y_{\bar{W}}^{n}=$ $\operatorname{Proj}\left(R\left(S_{\bar{W}}^{n}\right)\right)$. Here again the $\mu_{a}^{e}$ are local coordinates at the points $p_{a}^{e}$.

So now we have constructed from $S_{\bar{W}}^{n}$ a curve $Y_{W}^{n}$. Since $A_{X}$ is a subring of $S_{\bar{W}}^{n}$ and the filtration of $A_{X}$ is obtained by restriction of the filtration of $S_{\bar{W}}^{n}$ we have also an inclusion of $R\left(A_{X}\right)$ in $R\left(S_{\bar{W}}^{n}\right)$. This defines an $\ell$-fold branched covering map $f: Y_{\bar{W}}^{n} \rightarrow X$. The fiber of the point $p \in X$ consists of the points $p_{a}^{e}$ corresponding to one of the fields $F_{a}^{e}$. Since multiplication by $\lambda$ of an element $\sigma_{a}^{e}$ of $S_{W}^{n}$ with its highest order component in the blocks indexed by the support of the field $F_{a}^{e}$ increases the order by $\ell_{a}^{e}=\left[F_{a}^{e}: L_{\lambda}\right]$, the ramification index of $p_{a}^{e}$ is indeed $\ell_{a}^{e}$. The local coordinate $\mu_{a}^{e}$ satisfies clearly $\phi^{\#}\left(\lambda^{-1}\right)=\left(\mu_{a}^{e}\right)^{\ell}{ }_{a}^{e}$.

7.4. Now consider the element $W$ of $G r_{X}^{n}$. It is a finitely generated torsion free module over $S_{W}^{n}$ and hence defines a torsion free coherent sheaf over $\operatorname{Spec}\left(S_{W}^{n}\right)$. To extend it to $Y_{\bar{W}}^{n}$ we need a filtration on $W$ compatible with the filtration on $S_{\bar{W}}^{n}$. Recall from Sect. 6.6 that $S_{\bar{W}}^{n} \subset \bigoplus_{i=1}^{k} L_{\lambda}\left[P_{n_{i}}^{m_{i}}\right]$, with $n_{i}=m_{i} \ell_{i}$ and that the filtration of $S_{W}^{n}$ is obtained from this inclusion, see (5.5.6). Similarly the filtration on $W$ is obtained from the inclusion $W \subset L_{\lambda}^{n}$, where we decompose $L_{\lambda}^{n}=\bigoplus_{i=1}^{k} L_{\lambda}^{n_{2}}$ and introduce a filtration on it as follows. If $\left\{e_{0}^{(i)}, e_{1}^{(i)}, \ldots, e_{n_{1}-1}^{(i)}\right\}$ is the natural basis of the component $L_{\lambda}^{n_{2}}$, i.e., $e_{j}^{(i)}=e_{t}$, with $t=n_{1}+n_{2}+\cdots+n_{i-1}+j$, we give $e_{j}^{(i)}$ order $q_{j}$ where $j=q_{j} m_{i}+r_{j}, 0 \leqq r_{j}<m_{i}$ and within this block multiplication by 
$\lambda$ increases the order by $\ell_{i}$. One easily checks that this gives $L_{\lambda}^{n}=\bigoplus_{i=1}^{k} L_{\lambda}^{n_{i}}$ the structure of filtered $\bigoplus_{i=1}^{k} L_{\lambda}\left[\mathscr{P}_{n_{i}}^{m_{i}}\right]$-module.

Define then $F=\bigoplus F_{i}$, where $F_{i}=W_{i}$ are the elements of $W$ of order $i$ or less. Then $F$ is a graded, torsion free, finitely generated $R=R\left(S_{W}^{n}\right)$ module. It defines a torsion free coherent sheaf $\mathscr{F}$ on the curve $Y_{\bar{W}}^{n}$ in the usual way. The space of sections of $\mathscr{F}$ over $Y_{W}^{n}-f^{-1}(p)$ can then be identified with $W$, if we take as basis for the holomorphic stalks $\mathscr{F}_{p_{a}^{e}}$, thought of as free $\mathcal{O}_{Y_{W}^{n}, p_{a}^{e}}$ submodules of $L_{\lambda}$, the basis $\left\{e_{0}^{(i)}, e_{1}^{(i)}, \ldots, e_{m_{1}-1}^{(i)}\right\}_{i \in S_{a}^{e}}$. Note that in this manner we get as basis for $\mathscr{F}_{p_{a}^{e}}$ over $\mathcal{O}_{X, p}$ the elements

$$
\left\{e_{0}^{(i)}, e_{1}^{(i)}, \ldots, e_{m_{i}-1}^{(i)},\left(\lambda_{a}^{e}\right)^{-1} e_{0}=e_{m_{i}}^{(i)},\left(\lambda_{a}^{e}\right)^{-1} e_{1}=e_{m_{i}+1}^{(i)}, \ldots, e_{n_{i}-1}^{(i)}\right\}_{i \in S_{a}^{e}} .
$$

This uses the fact that

$$
\left(\lambda_{a}^{e}\right)^{-1} e_{j}^{(i)}=\left(\mathscr{P}_{n_{i}}^{m_{i}}\right)^{-1} e_{j}^{(i)}=e_{j+m_{i}}^{(i)}, \quad j<n_{i}-m_{i}
$$

In this way we have found also the sheaf $\mathscr{F}$ and the trivialization of it at the points in the inverse image of $p \in X$.

7.5. Let us summarize in the following theorem our construction of the inverse of the Krichever map of 5.4 .

Theorem 7.5.1. Let $X$ be a nonsingular curve, $p \in X$ a point, $\lambda^{-1}$ a local holomorphic coordinate at $p$ and $G r_{X}^{n}$ the Grassmannian (of rank $n$ bundles + trivializations) constructed from these data (see Sect. 2). Let $W \in G r_{X}^{n}$, let $\underline{n}$ be a partition $\left(n_{1}, n_{2}, \ldots, n_{k}\right)$ of $n$ and let $S_{\bar{W}}^{\underline{n}}$ be the finite order stabilizer algebra of $W$ of type $\underline{n}$ (Sect. 6), corresponding to the Heisenberg algebra $\mathscr{H}^{n}$ (Sect. 4). Let $\left\{F_{a}^{e}\right\}$ be the $k_{0}$ fields occurring in the decomposition of the tensor product $L_{\lambda} \otimes S_{\bar{W}}^{n}($ Sect. 6.6) and let $\ell_{a}^{e}$ be the degree of the field extension $F_{a}^{e}$ over $L_{\lambda}$, the field of Laurent series (Sect. 2.1).

Then there exists a curve $Y_{\bar{W}}^{n}$ and a covering map $f: Y_{\bar{W}}^{n} \rightarrow X$ such that $f^{-1}(p)$ consists of $k_{0}$ nonsingular points $p_{a}^{e}$, one for each field $F_{a}^{e}$, and such that the pull back divisor $f^{*}(p)$ is $\sum_{\ell} \ell_{a}^{e} p_{a}^{e}$.

Let $S_{a}^{e} \subset\{1,2, \ldots, k\}$ be the support of the field $F_{a}^{e}$ (Sect. 6.4). For each $i \in S_{a}^{e}$ the part $n_{i}$ of $\underline{n}$ is divisible by $\ell_{a}^{e}$, say $m_{i}=n_{i} / \ell_{a}^{e}$, and we put $m_{a}^{e}=\sum_{i \in S_{a}^{e}} m_{i}$. Then there exists an algebraic coherent torsion free sheaf $\mathscr{F}$ on $Y_{W}^{n}$ such that the stalk $\mathscr{F}_{p}$ is free of rank $m_{a}^{e}$ for each point $p_{a}^{e}$ in the inverse image of $p$. Furthermore there is a basis for each of the holomorphic stalks $\mathscr{F}_{p_{a}^{e}}^{\text {hol }}$ such that the space of algebraic sections of $\mathscr{F}$ over $Y_{\bar{W}}^{n}-f^{-1}(p)$, with respect to these bases, can be identified with the element $W$ in $G_{X}^{n}$ (Sect. 5.4).

\section{Time Evolution, Lax Equation, and Multi-component KP Equations}

8.1. In this section we study the flows on the Grassmannian by exponentiation of elements of $\mathscr{H}^{n}$. These flows leave invariant the commutative algebra $S_{W}^{n}$ and hence also the curve $\operatorname{Spec}\left(S_{W}^{n}\right)$. The flow on the Grassmannian gives a flow of modules over this ring which can be interpreted as a flow of bundles over the curve. In the case that the bundles have rank one, these flows are linear with respect to the toral structure of the Jacobian of the curve.

In Sect. 8.3 we relate these flows to multi-component KP equations by mapping $G r_{X}^{n}$ into the universal Grassmannian. Finally, in Sect. 8.4, we give an Hamiltonian interpretation of these flows on the cotangent bundle of the Grassmannian. 
8.2. Let $\sigma \in \mathscr{H}^{\underline{n}}$. Consider the flow on $G r_{X}^{n}$ given by:

$$
W(t)=\exp (t \sigma) W .
$$

Since for $W=g H_{X}^{(n, \text { hol })}$ we have that $S_{W}^{\text {hol }}=g\left(g l\left(n, A_{X}^{\text {hol }}\right)\right) g^{-1}$, it follows that

$$
S_{W(t)}^{\text {hol }}=\exp (t \sigma) g\left(g l\left(n, A_{X}^{\text {hol }}\right)\right) g^{-1} \exp (-t \sigma) .
$$

Thus if $s \in S_{W(t)}^{\text {hol }} \cap \mathscr{H}^{n}$ then, since $\mathscr{H}^{n}$ is commutative, it follows that $s=\exp (-t \sigma) \cdot s$ $\cdot \exp (t \sigma)$ is an element of $S_{W(0)}^{\text {hol }} \cap \mathscr{H}^{n}$, i.e., $S_{W(t)}^{\text {hol }} \cap \mathscr{H}^{\underline{n}}$ does not depend on the parameter $t$. In particular, the finite order part, $S_{\bar{W}(t)}^{n}$, is also independent of $t$, as is its completion $Y_{W}^{n}$. Thus the curve $\operatorname{Spec}\left(S_{W}^{n}\right)$ is independent of the flows. Furthermore the one parameter family of $S_{W}^{n}$ modules $W(t)$ corresponds to a one parameter family of bundles $E(t)$ over the curve $\operatorname{Spec}\left(S_{W}^{n}\right)$ as constructed in Chap. 7.

8.3. As we explained in Sect. 2.7 the Grassmannian $G r_{X}^{n}$ is a subvariety of the universal Grassmannian $G r=G r\left(H_{z}\right)$. When we are interested in flows on $G r_{X}^{n}$ determined by a partition $\underline{n}=\left(n_{1}, \ldots, n_{k}\right)$, of $n$ into $k$ parts, it is more convenient to map $G r_{X}^{n}$ into $G r\left(H_{z}^{k}\right)$, the Grassmannian of subspaces of $H_{z}^{k}$ whose projection to $H_{z,+}^{k}$ is Fredholm. Here we use the map $H_{\lambda}^{n} \rightarrow H_{z}^{k}$ given by

$$
\lambda^{l} e_{i} \mapsto z^{n_{a} l+j} \bar{e}_{a}
$$

where $i=n_{1}+\cdots+n_{a-1}+j$, and $e_{i}$ (respectively $\left.\bar{e}_{a}\right)$ give a basis for $\mathbb{C}^{n}\left(\right.$ resp. $\left.\mathbb{C}^{k}\right)$. (Of course the Grassmannians $\operatorname{Gr}\left(H_{z}^{k}\right)$ and $\operatorname{Gr}\left(H_{z}\right)$ are isomorphic.)

The action of the Heisenberg algebra $\mathscr{H}^{n}$ translates into an action of the algebra of diagonal $k \times k$ matrices on $H_{z}^{k}$ generated by $\Lambda_{a}^{i} \equiv z^{i} E_{a a}$, where $i \in \mathbb{Z}$ and $1 \leqq a \leqq k$. Indeed the map (8.3.1) induces the map $\mathscr{P}_{a} \mapsto z E_{a a}$, where the element $\mathscr{P}_{a}$ of $\mathscr{H}^{n}$ is defined in Sect. 4.1. Let $\Gamma^{n}$ denote the group of transformations on $H_{z}^{k}$ of the form $\phi^{\underline{n}}(t)=\exp \left(\sum_{i>0} \sum_{a=1}^{k} t_{i}^{a} \Lambda_{a}^{i}\right)$, where the $t_{i}^{a}$ are thought of as time variables. For $W \in \operatorname{Gr}\left(H_{z}^{k}\right)$, put $W(t)=\phi^{\underline{n}}(t)^{-1} W$. We say that $W(t)$ belongs to the big cell when the projection $W(t) \rightarrow H_{z,+}^{k}$ is an isomorphism, where $H_{z,+}^{k}$ is the subspace of $H_{z}^{k}$ of $k$-component vectors of the form $\sum_{i \geqq 0} f_{i} z^{i}, f_{i} \in \mathbb{C}^{k}$. If $W$ is in the big cell there is a unique $k \times k$ matrix wave function

$$
w_{W}(t)=\phi^{-n}(t)\left(1_{k \times k}+\sum_{i>0} w_{i} z^{-i}\right) \in W,
$$

where the $w_{i}$ are $k \times k$ matrix functions of the $t_{i}^{a}$, and we say that a $k \times k$ matrix belongs to $W$ if its columns do. We let $\bar{\partial}=\sum_{a=1}^{k} \overleftarrow{\partial}_{t_{1}^{a}}$ act as a differential operator on matrices from the right. More generally we consider $k \times k$ matrices with coefficients pseudo-differential operators in $\overleftarrow{\partial}$. Then we have

$$
\phi^{\underline{n}}(t)(\overleftarrow{\partial} E)=\phi^{\underline{n}}(t)(z E),
$$

for any $k \times k$ matrix $E$ (possibly dependent upon $t$ ). The wave operator is then the matrix pseudo-differential operator defined by

$$
\hat{w}=1_{k \times k}+\sum_{i>0} \overleftarrow{\partial}^{-i} w_{i},
$$

and we have

$$
w_{W}(t)=\phi^{\underline{n}}(t) \cdot \hat{w} .
$$


The wave operator $\hat{w}$ satisfies the equation

$$
\partial_{t_{i}^{a}} \hat{w}=-\hat{w}\left(R_{a}^{i}\right)_{-},
$$

where $R_{a}^{i}=\hat{w}^{-1} \cdot \grave{\partial}^{i}\left(E_{a a}\right) \cdot \hat{w}$ and the subscript - denotes the formal integral operator part of a pseudo-differential operator (i.e., the negative order part). The multi-component KP equation is then given by

$$
\partial_{t_{a}^{a}} Q=\left[Q,\left(R_{a}^{i}\right)_{+}\right],
$$

where $Q=\hat{w}^{-1} A \overleftarrow{\partial} \hat{w}$, for a constant diagonal matrix $A$ with distinct non-zero eigenvalues (cf. [DaJKM, UT, Di]). In [BtK] it is explained that with the multicomponent KP equation naturally are associated also difference equations (besides the differential equations (8.3.7)), coming from group elements commuting with the Heisenberg algebra. In this paper we will not discuss these discrete equations, which are in the simplest case the equations for the Toda-lattice.

Now let us assume that $W \in G r_{X}^{n}$ maps to an element of $G r\left(H_{z}^{k}\right)$ of index zero (this is not essential but will make the formulae much simpler). Then the flow on $G r_{X}^{n}$ from Sect. 8.2 will give rise to a multi-component KP flow on the image, $\bar{W}$, of $W$ in $\operatorname{Gr}\left(H_{z}^{k}\right)$. The elements of $\bar{W}$ coming from finite order elements of $W(t)$, i.e., those of the form $\phi^{\underline{n}}(t)\left(\sum_{-\infty}^{l} a_{i} z^{i}\right)$, generate a free rank $k$ module over the ring of differential operators. A basis for this module is given by the $k$ columns of the wave function (see $[\mathrm{DS}, \mathrm{SeW}, \mathrm{BtK}]$ ). This means that for any $f \in A_{X}$ (so $f W \subset W$ ) we have

$$
\bar{f} w_{W}(t)=w_{W}(t) P_{f},
$$

where $\bar{f}$ denotes $f$ realized as a matrix of size $k \times k$ acting on $H_{z}^{k}$, using 8.1.3, and $P_{f}$ is a $k \times k$ matrix of differential operators. The map $f \mapsto \bar{f} \mapsto P_{f}$ gives a map from $A_{X}$ to a commutative subring $\bar{A}_{X}$ of all $k \times k$ matrix differential operators. (For the simplest case of $X=\mathbf{P}^{1}$ and the principal Heisenberg algebra $(k=1)$ this ring is $\bar{A}_{X}=\mathbb{C}[L]$, the ring of polynomials in the Lax-operator $L$, the unique $n^{\text {th }}$ order differential operator such that $\lambda w_{W}=w_{W} L$.)

Similarly, we have, for any element $s$ of the stabilizer algebra $S_{W}^{n}$ of type $\underline{n}$ for $W \in G r_{X}^{n}$, a differential operator $P_{s}$. This gives a ring of commutative matrix differential operators $\bar{S}_{\bar{W}}^{n}$. Of course $\bar{A}_{X} \subset \bar{S}_{\bar{W}}^{n}$.

8.4. We now sketch how the Heisenberg flows on $G r_{X}^{n}$ are related to Hamiltonian flows on the duals of loop algebras and to Hamiltonian flows on the cotangent bundle of $G r_{X}^{n}$.

First note that the action of the group $G l\left(n, H_{\lambda}\right)$ on $G r_{X}^{n}$ lifts to an Hamiltonian action on $T^{*} G r_{X}^{n}$ in the usual way that any group action on a manifold lifts to an Hamiltonian action on the cotangent bundle. On the other hand, to give an Hamiltonian version of the Heisenberg flows on the dual of a loop algebra one must consider Lax pairs and the Adler, Kostant, Symes (AKS) theorem [Ad, Ko, Sy] (see also [FNR, RSTS]). This version of the flows works out completely only in the standard setting with $X=\mathbf{P}^{1}$, however we can make some comments on the more general situation.

We first discuss the case in which $X=\mathbf{P}^{1}$ so that $A_{X}^{\text {hol }}=H_{\lambda,+}$, the space of holomorphic functions on $\mathbf{P}^{1}-\infty$. Let $\sum t_{i}^{a} \mathscr{P}_{a}^{i}$ be an element of $\mathscr{H}^{n}$ and set $W(t)=\exp \left(-\sum t_{i}^{a} \mathscr{P}_{a}^{i}\right) W$. Assume that $W(t)$ belongs to the big cell of $G r_{\mathbf{P}^{1}}^{n}$, so that $W(t)=g_{-}(t) H_{\lambda,+}^{n}$, where $g(t)=\exp \left(-\sum t_{i}^{a} \mathscr{P}_{a}^{i}\right) g$ admits a Birkhoff decomposition 
of the form $g(t)=g_{-}(t) g_{+}(t)$ without diagonal term. Define the resolvents (cf. $[\mathrm{GD}])$ :

$$
R_{a}(t)=g_{-}^{-1}(t) \mathscr{P}_{a} g_{-}(t) .
$$

Let $R(t)=\sum A_{a} R_{a}$ for some choice of constants $A_{a} \in \mathbb{C}$. We have the Lax equation

$$
\partial_{t^{a}} R=\left[\left(R_{a}^{i}\right)_{+}, R\right]
$$

since $g_{-}^{-1} \partial_{f^{a}} g_{-}=-\left(R_{a}^{i}\right)_{-}$. Equation (8.4.2) can be realized as an Hamiltonian flow on the dual of the Lie algebra $g l\left(n, H_{\lambda,+}\right)$ with Hamiltonian function given by the restriction of an $\mathrm{Ad}^{*}$-invariant function on $g l\left(n, H_{\lambda}\right)^{*}$. Indeed, the splitting of $g l\left(n, H_{\lambda}\right)$ from Proposition 3.3.1, applied to the case $X=\mathbf{P}^{1}$, yields

$$
g l\left(n, H_{\lambda}\right)=g l\left(n, \lambda^{-1} \mathbb{C}\left\{\lambda^{-1}\right\}\right) \oplus g l\left(n, H_{\lambda,+}\right) .
$$

Using the pairing (3.3.6) this gives an identification

$$
\operatorname{gl}\left(n, H_{\lambda,+}\right)^{*} \sim \operatorname{gl}\left(n, \lambda^{-1} \mathbb{C}\left\{\lambda^{-1}\right\}\right) .
$$

If $F$ is an $\mathrm{Ad}^{*}$-invariant function on $g l\left(n, H_{\lambda}\right)^{*}$ we may restrict to $g l\left(n, H_{\lambda,+}\right)^{*}$ since (8.4.3) gives

$$
\operatorname{gl}\left(n, H_{\lambda}\right)^{*}=\operatorname{gl}\left(n, \lambda^{-1} \mathbb{C}\left\{\lambda^{-1}\right\}\right)^{*} \oplus \operatorname{gl}\left(n, H_{\lambda,+}\right)^{*} .
$$

Using the identification (8.4.4) the Hamiltonian flow for $F$ on $g l\left(n, H_{\lambda,+}\right)^{*}$ is given by the Lax pair

$$
\frac{d}{d t} A=\left[(d F(A))_{+}, A\right],
$$

where $A \in g l\left(n, \lambda^{-1} \mathbb{C}\left\{\lambda^{-1}\right\}\right)$ and $d F(A)$ is the differential at $A$ of $F$ as a function on $g l\left(n, H_{\lambda}\right)^{*}$, thought of as an element of $T_{A}^{*}\left(g l\left(n, H_{\lambda}\right)^{*}\right) \sim g l\left(n, H_{\lambda}\right)$. For example, if

$$
F_{i j}(A(\lambda))=\frac{1}{j} \oint \operatorname{tr}\left(\lambda^{i} A(\lambda)^{j}\right)
$$

then

$$
d F_{i j}(A(\lambda))=\lambda^{i} A(\lambda)^{j-1} .
$$

To realize (8.4.2) as a flow of this type we must first consider $R(t)$ as an element of $g l\left(n, \lambda^{-1} \mathbb{C}\left\{\lambda^{-1}\right\}\right)$. Since $R_{a}(t)$ has order 1 in $\lambda$ this is easily achieved by multiplying Eq. (8.4.2) by $\lambda^{-2}$. Next, we must find an Ad*-invariant function $F$ such that $d F\left(\lambda^{-2} R(t)\right)=R_{a}^{i}$. This is possible provided we choose the constants, $A_{a}$, distinct. Indeed, since $R$ is the conjugate of the matrix $\mathscr{P}=\sum A_{a} \mathscr{P}_{a}$ which is in block form, so that $R^{j-1}(t)$ is the conjugate of $\mathscr{P}^{j-1}=\sum A_{a}^{j-1} \mathscr{P}_{a}^{j-1}$, it is possible to find a linear combination, $F$, of the $F_{i j}$ 's which gives $d F\left(\lambda^{-2} R(t)\right)=R_{a}^{i}$.

Consider now the situation where there is a Laurent series $f(\lambda) \in L_{\lambda}$ such that $L(\lambda)=f(\lambda) R$ belongs to $g l\left(n, H_{\lambda_{+}}\right)$. Then we see that $s=f(\lambda) A_{a} \mathscr{P}_{a}$ belongs to $S_{W}^{\text {hol }} \cap \mathscr{H}^{n}$, the holomorphic stabilizer algebra of type $\underline{n}$ for $W$. Since from chapter 3 we have that $S_{W}^{\text {hol }}$ is contained in the cotangent fiber to $G r_{X}^{n}$ at the point $W$, we may consider the evolution of the pair $(W(t), L(\lambda)(t))$ as a flow through the point $(W(0), L(\lambda)(0))$ in the cotangent bundle $T^{*} G r_{X}^{n}$. For $X=\mathbf{P}^{1}$ this flow can be related to the AKS theorem and collective integrability by using a modification of the $\mathrm{Ad}^{*}$-invariant function used to give the Hamiltonian. This modification depends 
on the function $f(\lambda)$ and thus on the initial value, $W(0)$. Thus the flow for a particular Heisenberg element $\sigma$ on the whole of the Grassmannian does not arise in this way from a single Hamiltonian. However, this description of the flows does give a collective Hamiltonian version that generalizes to the case of a general base curve $X$. To justify these remarks we now give a brief excursion into the geometric proof of the AKS theorem (see e.g., [RSTS, GS2]).

Recall from Sect. 3 that the cotangent bundle $T^{*}(G / K)$ of the homogeneous space $G / K$ is symplectically equivalent to the Marsden-Weinstein reduction of $T^{*} G$ under the right action of $K$. We now consider Hamiltonian flows on this cotangent bundle arising from $\mathrm{Ad}^{*}$-invariant functions on $\mathrm{g}^{*}$.

If $f$ is a function on $\mathrm{g}^{*}$ we can pull it back via the moment map for the left action of $G$ on $T^{*} G$ to form a function $F$ on $T^{*} G$ which is invariant under the left action of $G$ on $T^{*} G$. In the case that $f$ is invariant under the $\mathrm{Ad}^{*}$ action then $F$ is also invariant under the right action of $G$ on $T^{*} G$, in particular, $F$ is invariant under the right $K$ action and so reduces to a function $\tilde{F}$ on $T^{*}(G / K)$.

To compute the Hamiltonian flow of $\tilde{F}$ on $T^{*}(G / K)$ we follow the prescription of [GS2] for computing flows for collective Hamiltonians: For $(g, \mu) \in G \times \mathfrak{g}^{*}$, let

$$
\xi=d f(\mu) \in\left(\mathfrak{g}^{*}\right)^{*} \simeq \mathfrak{g} .
$$

Then the Hamiltonian flow of $F$ through $(g, \mu)$ is given by

$$
(g, \mu) \rightarrow(\exp (t \xi) g, \mu) .
$$

Now, as in chapter 3, we let $J_{R, K}$ denote the moment map for the right $K$ action on $T^{*} G$, so that we may identify $T^{*}(G / K)$ with $J_{R, K}^{-1}(0) / K$. If $(g, \mu)$ is in $J_{R, K}^{-1}(0)$ then the flow for $\tilde{F}$ through the point $(g K, \mu) \in T^{*}(G / K)$ is just the projection of the above flow, i.e.

$$
(g K, \mu) \rightarrow(\exp (t \xi) g K, \mu) .
$$

If the algebra $g$ splits into two subalgebras, $\mathfrak{g}=\mathfrak{f} \oplus \mathfrak{L}$, then there is a left $L$ action on $T^{*}(G / K)$ with moment map

$$
(g K, \mu) \mapsto \mathrm{Ad}_{g}^{*} \mu \in \mathfrak{Q}^{*} .
$$

In this case, the Hamiltonian on $T^{*}(G / K)$ is just the pullback by this map of the restriction of $f$ to $\mathfrak{L}^{*}$. Thus the Lax pair flow for $f$ on $\mathfrak{L}^{*}$ given by the AKS theorem is just the image of the flow (8.4.10) under the moment map (8.4.11).

We now apply this construction to our situation where $G=G l\left(n, H_{\lambda}\right)$ and $K=G l\left(n, A_{X}^{\text {hol }}\right)$ so $\mathfrak{g}=g l\left(n, H_{\lambda}\right)$ and $\mathfrak{f}=g l\left(n, A_{X}^{\text {hol }}\right)$. Again, we begin with the case $X=\mathbf{P}^{1}$ so that $A_{\mathbf{P}^{1}}^{\text {hol }}=H_{\lambda,+}$ and (8.4.3) gives a splitting of $g l\left(n, H_{\lambda}\right)$ into two subalgebras. The above construction suggests we consider AKS flow on $\mathfrak{2}^{*}$ $=\operatorname{gl}\left(n, \lambda^{-1} \mathbb{C}\left\{\lambda^{-1}\right\}\right)^{*} \sim \operatorname{gl}\left(n, H_{\lambda,+}\right)$. In order that Eq. (8.4.2) be considered as a Lax pair flow on $g l\left(n, H_{\lambda,+}\right)$ we must find a function $f(\lambda)$ such that $L(\lambda)=f(\lambda) R(\lambda) \in g l\left(n, H_{\lambda,+}\right)$. In this case, $\quad s=g_{-} L(\lambda) g_{-}^{-1}=f(\lambda) \sum A_{a} \mathscr{P}_{a} \in$ $S_{W}^{\text {hol }} \cap \mathscr{H}^{n}$. If $F$ is the Ad*-invariant function on $g l\left(n, H_{\lambda}\right)^{*}$ which gives the flow (8.4.2) as an Hamiltonian flow on $g l\left(n, H_{\lambda,+}\right)^{*}$, then we must find a new Hamiltonian $\tilde{F}$ such that $d \tilde{F}(L(\lambda))=d F(R(\lambda))$. For instance, if $F=F_{i j}$ so $F(R(\lambda))=\oint \operatorname{tr}\left(\lambda^{i} R(\lambda)^{j}\right)$ then $\tilde{F}$ will be given by

$$
\tilde{F}(L(\lambda))=\oint \operatorname{tr}\left(f(\lambda) \lambda^{i}\left(f(\lambda)^{-1} L(\lambda)\right)^{j}\right) .
$$


Example (The non-linear Schrödinger flows). Take $n=2, \underline{n}=(1,1)$, and $X=\mathbf{P}^{1}$. We choose $W=g H_{X}^{(2, \text { hol })}=g H_{\lambda,+}^{2} \in G r_{X}^{2}$ and $s_{0} \in S_{\bar{W}}^{n}$. We assume that $W$ is in the "big cell" so that we may take $g=g_{-} \in L g l_{-}(n, \mathbb{C})$. Then $s_{0}=g_{-} L(\lambda) g_{-}^{-1}$ for some matricial polynomial $L(\lambda)$, by Lemma 3.3.4, (6.1.2), and the fact that $A_{X}=\mathbb{C}[\lambda]$. Since the leading order term in $g_{-}$is the identity matrix, $s_{0}$ and $L(\lambda)$ must have the same leading order terms. Assume this to be $\lambda^{k}\left(\begin{array}{cc}i & 0 \\ 0 & -i\end{array}\right)$. Furthermore assume that $L(\lambda)$ is traceless. Then $s_{0}$ is also traceless and hence has the form

$$
s_{0}(\lambda)=p(\lambda)\left(\begin{array}{cc}
i & 0 \\
0 & -i
\end{array}\right)
$$

where $p(\lambda)=\lambda^{k}+p_{k-1} \lambda^{k-1}+\ldots$ is in $\mathbb{C}\left(\left(\lambda^{-1}\right)\right)$. Then we have

$$
\frac{1}{2} d\left(F_{k, 2}-p_{k-1} F_{k-1,2}\right)\left(s_{0}\right)=\lambda\left(\begin{array}{cc}
i & 0 \\
0 & -i
\end{array}\right)+O\left(\lambda^{-1}\right),
$$

and

$$
\frac{1}{2} d\left(F_{k+1,2}-p_{k-1} F_{k, 2}-\left(p_{k-2}-p_{k-1}^{2}\right) F_{k-1,2}\right)\left(s_{0}\right)=\lambda^{2}\left(\begin{array}{cc}
i & 0 \\
0 & -i
\end{array}\right)+O\left(\lambda^{-1}\right) .
$$

These give the $x$ and $t$ flows respectively on $G r_{X}^{2}$ that give rise to solutions of the non-linear Schrödinger equation. So we see in this example that the Hamiltonians for the flows in the cotangent bundle to the Grassmannian depend on the initial condition (viz., $s_{0}$ ) chosen in the fiber.

Finally we remark that when $X$ is more general than $\mathbf{P}^{1}$, the Lie algebra splitting from chapter 3 is not a splitting into two subalgebras but only into a subalgebra plus a linear subspace. Thus we cannot apply the AKS theorem in this setting. However, the Heisenberg flows may still be realized as Hamiltonian flows on $T^{*} G r_{X}^{n}$ with Ad*-invariant Hamiltonian, as in Eq. (8.4.11). Indeed, if we choose a flow generator $\sigma \in \mathscr{H}^{n}$, it is then necessary to find $s \in S_{W}^{n}$ and an Ad*-invariant function $f$ on $g l\left(n, H_{\lambda}\right)^{*}$ so that the Hamiltonian flow for $f$ through $(W, s) \in T^{*} G r_{X}^{n}$, given by (8.4.11) is just $(\exp (t \sigma) W, s)$. This is achieved by finding $f$ and $s$ so that $(d f(s))_{+}=\sigma$. This may not be possible for all choices of $\sigma \in \mathscr{H}^{\underline{n}}$ but since $s \in \mathscr{H}^{n}$ it follows that $d f(s) \in \mathscr{H}^{\underline{n}}$ as well so that at least some of the Heisenberg flows may be realized in this way.

\section{Remarks}

9.1. According to Theorem 7.5.1 we obtain for every point $W$ of $G r_{X}^{n}$ and every choice of Heisenberg algebra, i.e., for every choice of partition $\underline{n}$ of $n$, a curve $Y_{\bar{W}}^{n}$ covering $X$. We have not discussed the relation between these curves for the various choices of Heisenberg algebra. In fact this is rather mysterious to us. The first idea that one has is that somehow the curves corresponding to a single $W$ should be the same. This can however not be literally true since for the principal Heisenberg algebra one obtains always an irreducible curve while, as we have seen in Sect. 6.4, for other Heisenbergs one can get reducible curves. Also note that if we consider the flows $W \mapsto W(t)$ of type $\underline{n}$ on $G r_{X}^{n}$, i.e., the flows corresponding to the Heisenberg algebra $\mathscr{H}^{\underline{n}}$, the curve $Y_{W}^{n}$ remains invariant, while the curves obtained from the choice of other Heisenbergs will change. 
9.2. In Sect. 8 we derived differential equations coming from Heisenberg flows on the Grassmannian by embedding $G r_{X}^{n}$ into a universal Grassmannian, thereby exhibiting these equations as subsystems of multicomponent KP systems.

Now in case $X=\mathbf{P}^{1}$ the equations corresponding to the various Heisenbergs can be formulated entirely in terms of the representation theory of the loop algebra of $g l(n, \mathbb{C})(\mathrm{cf}$. [KaW, BtK]). These representations involve a decomposition of the loop algebra in elements containing only positive or only negative powers of $\lambda$, i.e. the decomposition of Proposition 3.3.1 for $X=\mathbf{P}^{1}$. One can also formulate these equations in terms of Lax equations on the dual of the loop algebra, again using the decomposition in positive and negative parts related to the Riemann sphere.

This makes one think that it should be possible to formulate the equations of Sect. 8 intrinsically in terms of constructions related to the Riemann surface $X$, without using the embedding into a universal Grassmannian. However there are a lot of things apparently still unknown. For instance the representation theory seems not to be developed yet (see however the papers [CEH, Che, DJM]). One way of constructing representations related to $X$ would be to consider the sections of a determinant bundle over $\mathrm{Gr}_{X}^{n}$ as in for instance [PrS] for the case of $X=\mathbf{P}^{1}$. In [Br] noncommutative theta functions related to vector bundles on curves are constructed using this determinant bundle. The connection to differential equations remains to be worked out. If one wants to get Lax equations on the dual of the loop algebra related to the curve $X$ one runs into the problem that the decomposition of Proposition 3.3.1 is not, as is usual in AKS theory, into two subspaces but that there is a finite dimensional piece in the middle. Lax type equations coming from such decompositions into three subspaces have been studied in [Mi]. Of course there is also the theory of Poisson-Lie groups, $R$ matrices etc. (see e.g., [Lu, LuW, STS1, STS2]) that could be developed in the context of $\mathrm{Gr}_{X}^{n}$, see alo [KS].

9.3. The Grassmannian $G r_{X}^{n}$ that we have used in this paper depended on choosing a point $p$ on the Riemann surface $X$. Of course it is possible to extend the theory by choosing a finite number of points $\left\{p_{i}\right\}$ on $X$, so that the elements of the generalized Grassmannian consists of equivalence classes of vector bundles on $X$ together with trivializations at $\left\{p_{i}\right\}$. To obtain an inverse Krichever map analogous to the one described in Theorem 7.5.1 one should then choose at every point in $\left\{p_{i}\right\}$ an Heisenberg algebra. The case of two points is relevant for the study of harmonic maps. For instance in [Hi2] one can find that both the homogeneous and the principal Heisenberg algebra of the loop group of $\operatorname{sl}(2, \mathbb{C})$ are relevant for the construction of all harmonic maps from the 2-torus to the 3-sphere, using spectral curves.

Acknowledgements. We would like to thank Robert Varley, Kevin Clancey, Mitchell Rothstein, and Emma Previato for interesting and instructive discussions.

\section{References}

[AB] Adams, M.R., Bergvelt, M.J.: Heisenberg algebras, Grassmannians and isospectral curves, The Geometry of Hamiltonian Systems, MSRI Publication 22, Ratiu, T. (ed.) Berlin, Heidelberg, New York: Springer 1991, pp. 1-8

[AHH] Adams, M.R., Harnad, J., Hurtubise, J.: Coadjoint orbits, spectral curves and Darboux coordinates. The Geometry of Hamiltonian Systems, MSRI Publication 22, Ratiu, T. (ed.) Berlin, Heidelberg, New York: Springer 1991, pp. 9-22 
[Ad] Adler, M.: On a Trace Functional for Formal Pseudo-Differential Operators and the Symplectic Structure of the Korteweg-de Vries Equation. Invent. Math. 50, 219-248 (1979)

[ADKP] Arbarello, E., De Concini, C., Kac, V., Procesi, C.: Moduli spaces of curves and representation theory. Commun. Math. Phys. 117, 1-36 (1988)

[AtB] Atiyah, M.F., Bott, R.: The Yang-Mills equations over Riemann surfaces. Phil. Trans. R. Soc. Lond. A 308, 523-615 (1982)

[B] Beauville, A.: Jacobiennes des courbes spectrales et systemes Hamiltoniens completement integrables. Preprint (1989)

[BNR] Beauville, A., Narasimhan, M.S., Ramanan, S.: Spectral curves and the generalized theta divisor. J. fur die reine und angewandte Math. 398, 169-179 (1989)

[BtK] Bergvelt, M.J., ten Kroode, A.P.E.: Partitions, vertex operator constructions and multicomponent KP equations. Preprint (1991)

[Bo] Bourbaki, N.: Commutative algebra. Berlin, Heidelberg, New York: Springer 1989

[Br] Brylinski, J.L.: Loop groups and non-commutative theta functions. Preprint (1989)

[CEH] Carey, A.L., Eastwood, M.G., Hannabus, K.C.: Riemann surfaces, Clifford algebras and infinite dimensional groups. Commun. Math. Phys. 130, 217-236 (1990)

[Che] Cherednik, I.V.: Definitions of functions for affine Lie algebras. Funct. Anal. Appl. 17, 243 (1983); On the group-theoretical interpretation of Baker functions and $\tau$-functions. Russ. Math. Surveys 38-6, 113-114

[DaJKM] Date, E., Jimbo, M., Kashiwara, M., Miwa, T.: Transformation groups for soliton equations. Proceedings of RIMS symposium, Jimbo, M., Miwa, T. (eds.) Singapore: World Scientific 1983, pp. 39-120

[DaJM] Date, E., Jimbo, M., Miwa, T.: Landau-Lifshitz equation. J. Phys. Soc. A 1983, 388-393 (1983)

[Di] Dickey, L.A.: Another example of a $\tau$-function. Proceedings of the Workshop on Hamiltonian systems. Montreal (1989) Harnad, J., Marsden, J.E. (eds.) Les Publications CRM, Montreal, 1990, pp. 39-44; On the $\tau$-functions of matrix hierarchies of integrable equations; On Segal-Wilson's definition of the $\tau$-function and hierarchies AKNS-D and mcKP

[DS] Drinfeld, V.G., Sokolov, V.V.: Lie algebras and equations of KdV-type. J. Sov. Math. 30, 1975-2036 (1985)

[Du] Dubrovin, B.A.: Theta Functions and Nonlinear Equations. Russ. Math. Surveys 35, 11-92 (1981)

[FNR] Flaschka, H., Newell, A.C., Ratiu, T.: Kac Moody algebras and soliton equations, II, Lax equation associated with $A_{1}^{(1)}$. Physica 9D, 300 (1983)

[GD] Gelfand, I.M., Dickey, L.A.: Fractional powers of operators and Hamiltonian systems. Funct. Anal. Appl. 10, 259-273 (1976); The resolvent and Hamiltonian systems. Funct. Anal. Appl. 11, 93-105 (1977)

[GH] Griffiths, P., Harris, J.: Principles of Algebraic Geometry. New York: Wiley 1978

[GS1] Guillemin, V., Sternberg, S.: Symplectic Techniques in Physics. Cambridge: Cambridge University Press 1984

[GS2] Guillemin, V., Sternberg, S.: On the method of Symes for integrating systems of the Toda type. Lett. Math. Phys. 7, 113-115 (1983)

[H] Harder, G.: Eine Bemerkung zu einer Arbeit von P.E. Newstaed, J. fur Mathematik 242, 16-25 (1970)

[Ha] Hartshorne, R.: Algebraic geometry. Berlin, Heidelberg, New York: Springer 1977

[Hi] Hitchin, N.: Stable bundles and integrable systems. Duke Math. J. 54, 91-114 (1987)

[Hi2] Hitchin, N.: Harmonic maps from the 2-torus to the 3-sphere. J. Diff. Geom. 31, $627-710(1990)$

[Ka] Kac, V.G.: Infinite dimensional Lie algebras. Second edition, Boston: Cambridge University Press 1985

[KaP] Kac, V.G., Peterson, D.H.: 112 constructions of the basic representation of the loopgroup of $E_{8}$, Proceedings of the symposium on anomalies, geometry and topology, Singapore: World Scientific 1985, pp. 276-298

[KaW] Kac, V.G., Wakimoto, M.: Exceptional hierarchies of soliton equations. Theta functions, Bowdoin 1987, vol. 49, Ehrenpreis, L., Gunning, R.C. (eds.) Proceedings of symposia in pure mathematics. Am. Math. Soc., 1989, pp. 191-237 
[KaSU] Katsura, T., Shimizu, Y., Ueno, K.: New Bosonization and Conformal Field Theory over Z. Commun. Math. Phys. 121, 603-627 (1989)

[KNTY] Kawamoto, N., Namikawa, Y., Tsuchiya, A., Yamada, Y.: Geometric realization of conformal field theory on Riemann surfaces. Commun. Math. Phys. 116, 247-308 (1988)

[KdV] Korteweg, D.J., de Vries, G.: On the change of form of long waves advancing in a rectangular canal, and a new type of long stationary waves. Phil. Mag. 39, 422-443 (1895)

[KS] Kossmann-Schwarzbach, Y.: Quasi-bigebres de Lie et groupes de Lie quasi-Poisson. C.R. Acad. Sci. Paris 312, Serie I, 391-394 (1991)

[Ko] Kostant, B.: The solution of the generalized Toda lattice and representation theory. Adv. Math. 34, 195-338 (1979)

[Kr] Krichever, I.M.: Methods of algebraic geometry in the theory of nonlinear equations. Russ. Math. Surv. 32, 185-213 (1977)

[KrN] Krichever, I.M., Novikov, S.P.: Holomorphic Bundles over Algebraic Curves and Nonlinear Equations. Russ. Math. Surv. 35 (1980)

[tKr] ten Kroode, A.P.E.: Affine Lie algebras and integrable systems. Thesis, University of Amsterdam, 1988

[Ku] Kunz, E.: Introduction to commutative algebra and algebraic geometry. Boston: Birkhäuser 1985

[LR] Landi, G., Reina, C.: Symplectic dynamics on the universal Grassmannian and algebraic integrability. Preprint (1991)

[Lep] Lepowsky, J.: Calculus of vertex operators. Proc. Natl. Acad. USA 82, 8295-8299 (1985)

[Lu] Lu, J.H.: Poisson and symplectic structures of a Poisson Lie group. Preprint (December 1988)

[LuW] Lu, J.H., Weinstein, A.: Poisson Lie groups, dressing transformations, and Bruhat decompositions. J. Diff. Geom. 31, 501-526 (1990)

[McK] McKean, H.P.: Is there an infinite-dimensional algebraic geometry? Hints from KdV, Proc. Symp. in Pure Mathematics, vol. 49, Providence, RI: Am. Math. Soc., 1989, pp. 27-37

[Mi] Mikhalev, V.G.: A generalization of the Kac-Moody algebras with a parameter on an algebraic curve and perturbations of solitons. Commun. Math. Phys. 134, 633-646 (1990)

[vMM] van Moerbeke, P., Mumford, D.: The Spectrum of Difference Operators and Algebraic Curves. Acta Math. 143, 93-154 (1979)

[Mul1] Mulase, M.: Cohomological structure of soliton equations and Jacobian varieties. J. Diff. Geom. 19, 403-430 (1984)

[Mul2] Mulase, M.: Category of vector bundles on algebraic curves and infinite dimensional Grassmannians. Int. J. Math. 1, 293-342 (1990)

[Mum1] Mumford, D.: An algebro-geometric construction of commuting operators and of solutions to the Toda lattice equation, Korteweg de Vries equation and related non-linear equations. Intl. Symp. on Algebraic Geometry Kyoto, 1977, pp. 115-153

[Mum2] Mumford, D.: Tata lectures on theta, I, II. Boston: Birkhäuser 1983

[PrS] Pressley, A., Segal, G.: Loop groups. Oxford: Clarendon Press 1986

[PrW] Previato, E., Wilson, G.:. Vectorbundles over curves and solutions of the KP equations. Theta functions, Bowdoin 1987, vol. 49, Ehrenpreis, L., Gunning, R.C. (eds.) Proceedings of symposia in pure mathematics. Providence, RI: Am. Math. Soc., 1989, pp. 553-569

[R] Read, A.H.: A converse of Cauchy's theorem and applications to extremal problems. Acta Math. 100, 1-21 (1958)

[RSTS] Reiman, A.G., Semenov-Tian-Shansky, M.A.: Reduction of Hamiltonian systems, Affine Lie algebras and Lax Equations I, II. Invent. Math. 63, 423-432 (1981)

[Ro] Royden, H.L.: The boundary values of analytic and harmonic functions. Math. Zeitschr 78, 1-24 (1962)

[Sa] Sato, M.: Soliton equations as dynamical systems on infinite dimensional Grassmann manifolds. RIMS Kokyoroku 439, 30-46 (1981)

[SeW] Segal, G.B., Wilson, G.: Loop groups and equations of KdV type. Publ. Math. I.H.E.S. 61, 5-65 (1985) 
[STS1] Semenov-Tyan-Shanskii, M.A.: Poisson groups and dressing transformations. J. Sov. Math. 46, 1641-1657 (1989)

[STS2] Semenov-Tyan-Shanskii, M.A.: What is a classical $r$-matrix? Funct. Anal. Appl. 17(4), 259-272 (1983)

[Ser] Serre, J.P.: Géométrie Algébrique et Géométrie Analytique. Collected Works, Volume 1, Berlin, Heidelberg, New York: Springer 1986, pp. 402-443

[Sh] Shiota, T.: Characterization of Jacobians in terms of soliton equations. Inventiones 83, 333-382 (1986)

[Si] Simha, R.R.: The Behnke-Stein theorem for open Riemann surfaces. Proc. AMS 105, 876

[Sy] Symes, W.W.: Systems of Toda type, inverse spectral problems and representation theory. Inv. Math. 59, 13-51 (1980)

[UT] Ueno, K., Takasaki, K.: Toda Lattice Hierarchy. Advanced Studies in Mathematics 4, $1-95(1984)$

Communicated by S.-T. Yau 
